\title{
Development of Acoustic Metamaterials for Efficient Sound Control and Noise Mitigation
}

A Dissertation

Presented to

the Faculty of the Graduate School

University of Missouri-Columbia

In Partial Fulfillment

of the Requirement for the Degree

Doctor of Philosophy

\section{By}

Huy Nguyen

Dissertation Supervisor: Dr. Guoliang Huang

July 2021 
The undersigned, appointed by the dean of Graduate School have examined the dissertation entitled

\section{Development of Acoustic Metamaterials for Efficient Sound Control and Noise Mitigation}

Presented by Huy Nguyen, a candidate for degree of doctor of philosophy, and hereby certify that, in their opinion, it is worthy of acceptance.

Professor Guoliang Huang

Professor Gary Solbrekken

Professor Qingsong Yu

Professor Zheng Yan

Professor Sarah Orton 


\section{Acknowledgement}

This thesis would not have been possible to complete without the support, encourage and guidance of many people. First and foremost, I would like to express my sincere thanks to my supervisor, Dr. Guoliang Huang, for the opportunities and support he has given me during my Ph.D. program at Mizzou. His encouragement, patience, and guidance allowed me to have a pleasant and fulfilling research experience for the past seven years and will not be forgotten.

I would like to thank professors Gary Solbrekken, Qingsong Yu, Zheng Yan, and Sarah Orton for being part of my thesis committee, patiently answering my many emails, and dedicating some of their time.

Special thanks go to Dr. Sharon Tracy for our collaborations on some of the projects presented in the dissertation. As a great project manager, she has given us many critical suggestions on our research. We have always enjoyed the project meetings with her.

I am grateful for the numerous discussions with all the current and former members of Dr. Huang's group, making my $\mathrm{Ph}$. D. experience fruitful and fun. I also highly appreciate the help provided by Dr. Yangyang Chen, Dr. Hui Chen, Dr. Xian Chen, Dr. Miles V. Barnhart, Qian $\mathrm{Wu}$, and Yukai $\mathrm{Yu}$ on some discussion, simulation, sample fabrication, and experimental testing.

The financial supports from the scholarship agency, Vietnam International Education Development Steelcase (VIED), and Steelcase Inc. are also gratefully acknowledged.

Finally, I would like to thank my wife, daughter, son, and parents for their encouragement and love during this journey. 


\section{Table of Contents}

Acknowledgement ........................................................................................................................ ii

List of Figures......................................................................................................................... $\mathrm{v}$

List of Tables ..................................................................................................................

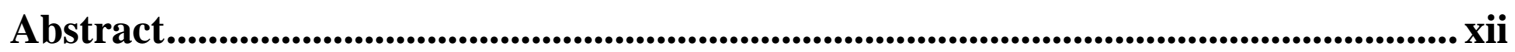

Chapter 1. Introduction ............................................................................................... 1

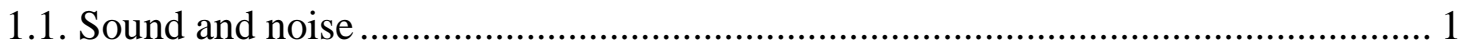

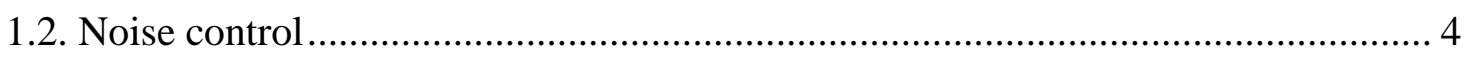

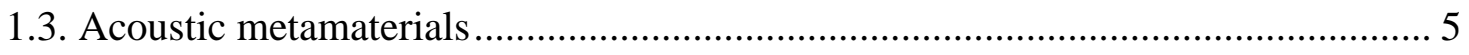

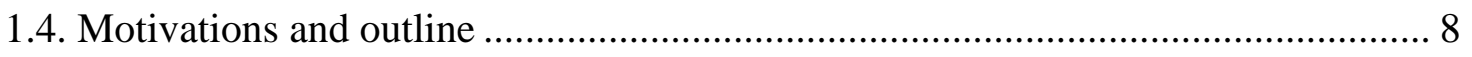

Chapter 2. Membrane-type Harvesting Acoustic Metamaterials .................................. 10

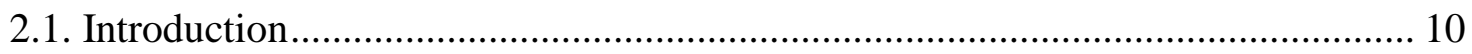

2.2. Analytical modeling of the Magneto-based Acoustic Metamaterial Harvester.... 13

2.2.1. The determination of magnetic force ........................................................... 15

2.2.2. Coupling Vibro-Acoustic-Electromagnetic model of the MAM .................... 18

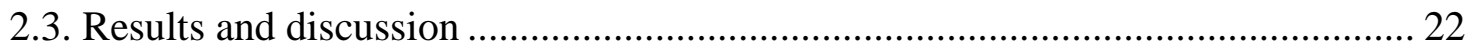

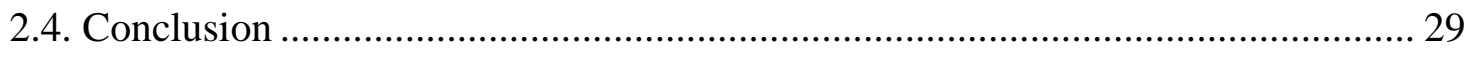

Chapter 3. Membrane-type Acoustic Metamaterial Panel ............................................... 31

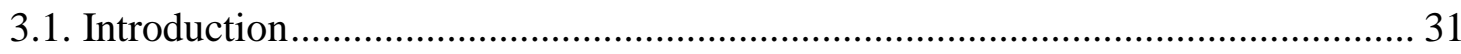

3.2. Double layer acoustic metamaterial unit-cell design ............................................. 33

3.3. Acoustic metamaterial panel.............................................................................. 39

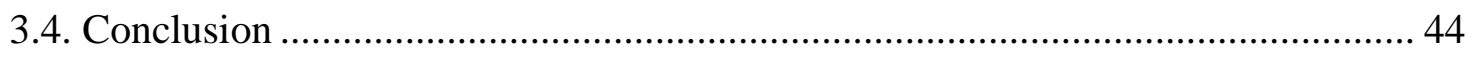

Chapter 4. Sonic Crystal Based Acoustic Barrier................................................................. 46 


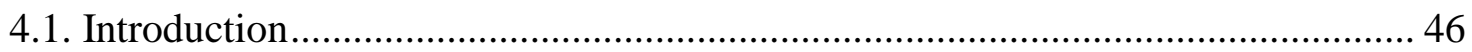

4.2. Theoretical formulation of Fano-based metamaterials .................................... 49

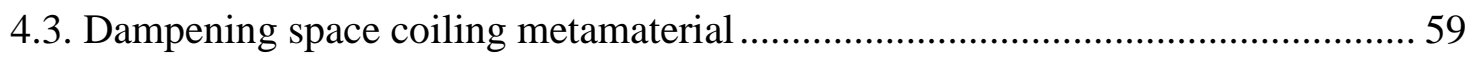

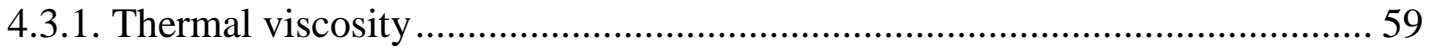

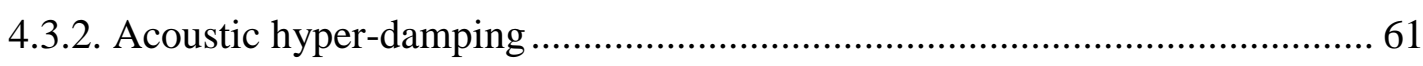

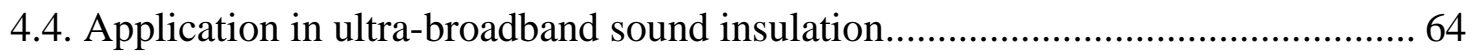

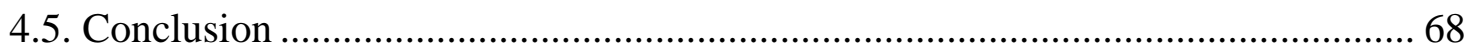

Chapter 5. Helmholtz Resonator Based Acoustic Silencing...................................... 69

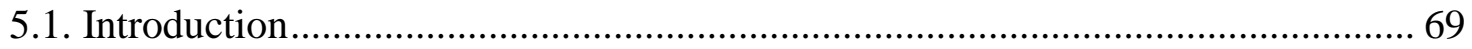

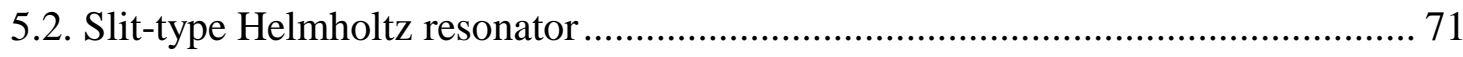

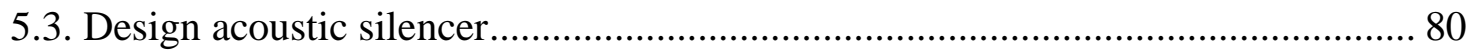

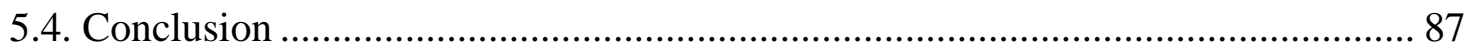

Chapter 6. Conclusion .................................................................................................................... 89

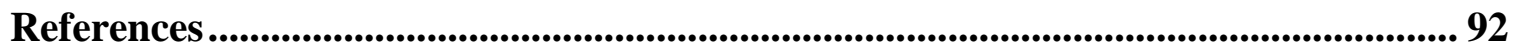

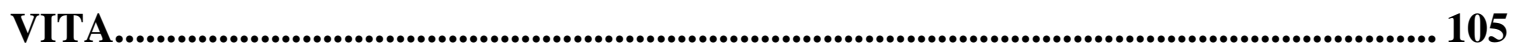




\section{List of Figures}

Figure 1.1. The illustration of sound wave propagating through air particles. 1

Figure 1.2. The equal loudness contours and the SPL spectrum of some popular noise sources as illustrated by the insets. 2

Figure 1.3. (a) The transmission performance of MAM unit cell (red) and single panel (blue) [2]. (b) The electromotive force induced inside the harvesting circuit [4]. (c) The transmission loss performance of individual resonators and silencer [8]. (d) The performance of barriers comprised of Mie resonators [9] 6

Figure 2.1. (a) - the model of the MAM harvester, (b) - the mechanical equivalent of the electromagnetism part of the MAM harvester, (c) - the model of the permanent magnet coin. 14

Figure 2.2. The comparison between the simulation and analytical results for the cases the membrane with dissipative property but without effect of the magnet, the membrane without dissipative property but with the magnet effect, the membrane with both dissipative property and the magnet effect.

Figure 2.3. The effect of the external load on the conversion (a) and harvesting (b) coefficients. 26

Figure 2.4. The effect of the membrane's thickness on the conversion coefficient of (a) the harvester and the elastic dissipative energy of (b) the membrane. 27

Figure 2.5. The effect of the mass' dimensions on the conversion coefficient of the harvester. 28 
Figure 2.6. (a) - The effect of the membrane's prestress on the conversion coefficient; (b) - The optimized harvesting coefficient of the harvester when $\sigma 0=0.5 \times 105 \mathrm{~N} / \mathrm{m} 2 \ldots \ldots 28$

Figure 3.1. (a) Schematic of the proposed large-scale acoustic panel design. The incident sound ( $p i)$ wave is mostly reflected $(p r)$, resulting in strongly suppressed sound transmission ( $p t$ ). (b) Detailed view of the double-layer membrane-type acoustic metamaterial unit cell. It is assembled from two membrane-type acoustic metamaterial unit cells and the gridded-like elastic frames. (c) Sectional view of the frame. All the geometric parameters are indicated. The parts of different colors are all made of aluminum. (d) Schematic of the acoustic model of a double-layer membrane-type acoustic metamaterial unit cell in the tube environment. An incident sound wave of normalized amplitude is considered. $T d$ and $R d$ denote the transmitted and reflected waves, respectively. 33

Figure 3.2. (a) Transmission loss spectrum of the MAM/DMAM unit cells from both simulation (Num.) and theoretical (Anal.) analysis. (b) Mode shapes of the MAM/DMAM unit cell at the peaks and dips. 36

Figure 3.3. (a) - Transmission loss spectra of the single MAM unit cell under different membrane pre-tension conditions (r) and the DMAM unit cell comprised from two identical MAMs under a pre-tension of $5 \mathrm{MPa}$. Comparison of TL spectra of (b) MAM panels and (c) DMAM panels with different thicknesses of the panel thickness when a pretension of $5 \mathrm{MPa}$ is employed on the membranes. (d) The mode shapes of the DMAM panel of the highlighted peaks and dips. 38

Figure 3.4. (a) Schematic illustration of the cut away DMAM panel frame. (b) Exploded view of the DMAM panel frame 40 
Figure 3.5. (a) Photograph of stretching the membrane in the acoustic panel. The pretension on the membrane can be easily adjusted. (b) Photograph of the large-scale double-layer membrane-type acoustic metamaterial-based panel. (c) Schematic illustration of the experimental setup. The foam layer within the tube cross section is of a thickness of 8 inches. A 2 inch-thick air cavity is arranged next to the foam. (d) Comparison between the simulation and experimental results for the sample shown in (b). The noise reduction performance according to the conventional mass density law is also presented for illustrating how well the large-scale double-layer membrane-type acoustic metamaterialbased panel performs. 42

Figure 3.6. (a) Setup for measuring absorption coefficient in a commercial impedance tube. (b) The experimental and simulation results. 44

Figure 4.1. (a) Schematic illustration of the acoustic barrier assembled from SCMs. It efficiently blocks the incoming sound waves (pi) indicated by the blue arrows. The substantially reflected $(p r)$ and heavily suppressed transmitted $(p t)$ sound fields are indicated by red and green arrows, respectively. (b) Sectional view of the cross-section of an SCM unit cell.

Figure 4.2. Schematic of an effective SCM coupling unit cell submerged in an impedance tube. $p i, p r$ and $p t$ represent the incident, reflected and transmitted pressure fields, respectively. 50

Figure 4.3. (a) TL spectra of the effective SCM unit cell. Theoretical and numerical results are shown, together with the numerical result of the actual SCM unit cell. At the two TL peaks, both the magnitude and phase of the velocity distributions of the effective SCM 
coupling unit are illustrated as insets. (b) Frequency-dependent phase spectra of the normalized volume velocities $v$ at the outlet of the channels. (c) Frequency-dependent amplitude spectra of the normalized volume velocities $v$ at the outlet of the channels. . 55

Figure 4.4. The transmission loss spectrum from analytical solution and numerical simulation in three cases: only ventilation channels, only effective channel and both of them presence on the SCM unit cell. 56

Figure 4.5. (a) Real part of the normalized effective mass density $\rho e f f *=\operatorname{Re} \rho e f f / \rho 0$ and the bulk modulus $\mathcal{B}$ eff $*=\operatorname{Re} \mathcal{B}$ eff $\mathcal{B} 0$. (b) The decaying factor of the unit cell $\gamma e f f *$ $=-$ Imkeffa. Two single-negative bands are highlighted in grey. 58

Figure 4.6. (a) Diagram of the deployment of viscous foams. All foam layers have identical thickness $t f$. (b) Velocity and pressure profiles along the effective channel at its monopolar and dipolar modes. (c) TL spectra of the actual SCM with different configurations of the space-coiling channel: without thermal viscosity, with thermal viscosity and with dampening foam layers of thicknesses $1 \mathrm{~mm}, 3 \mathrm{~mm}$ and $5 \mathrm{~mm}$. (d) The pressure and velocity fields at the monopole and dipole in three cases: lossless, viscous and $5 \mathrm{~mm}$ foam employed. The velocity representing the strength of the resonances is heavily dampened in the case of viscous foam employed, so it is called 'acoustic hyper-damping'. 61

Figure 4.7. (a) The setup of experiment to determine the effective parameter of acoustic foam. An impedance tube with two condense microphones are used to measure the absorption of the foam. (b) The absorption from experiment and fitting model.

Figure 4.8. (a) Schematic of the acoustic barrier wall comprising three space-coiling cells with the same open ratio but different channel widths. (b) Zoom-in views of the super-cell 
bar without and with the dampening foam layers. (c) Numerically obtained transmission loss spectra of the lossless individual unit cells (blue, red and yellow curves with symbols), lossless super-cell (purple solid) and dampened super-cell (green dashed). 66

Figure 4.9. (a) Schematic illustration of the simulation model under oblique incidence. Top and bottom of the ribbon are defined by Floquet periodic boundary conditions. (b) Transmission loss (TL) spectra of the barrier at various angles of incidence. 66

Figure 4.10. (a) Sectional view of the impedance tube and the samples in the experiment. The inset shows the detailed view of the dampened super-cell. (b) Comparison of the transmission loss (TL) and absorption (A) spectra between simulation and experiment results for the dampened super-cell.

Figure 5.1. (a) Sectional view of a side-branched SHR. The blue arrows correspond to the incoming acoustic waves. (b) Schematic of a SHR with all the involved geometrical parameters indicated. The SHR is divided into multiple domains, labeled as A, B, C, and D. 71

Figure 5.2. (a) and (b) TL spectra of non-viscous and viscous SHRs, respectively. The dashed curves correspond to analytical results, whereas the solid ones represent the simulation results.

Figure 5.3. (a) and (b) Sectional views of a single-layer acoustic silencer consisting of five labeled SHRs and the double-layer acoustic silencer containing two single-layer acoustic silencers. (c) TL spectra of the non-viscous (NVC) and viscous (VC) single-layer acoustic silencers from analytical and numerical calculations. (d) Comparison between the viscous 
single-layer and double-layer silencers and the detuned viscous double-layer silencer. Here, S and A correspond to simulation and analytical results, respectively.................. 80

Figure 5.4. (a) Photograph of the experimental setup including a Mecanum Inc. standard impedance tube and a detuned double-layer acoustic silencer sample [see the inset]. (b) Comparison between the numerical (solid curve) and the experimental results (dashed curve) of the detuned double-layer acoustic silencer design. ......................................... 86 


\section{List of Tables}

Table 2.1. The MAM harvester's parameters.

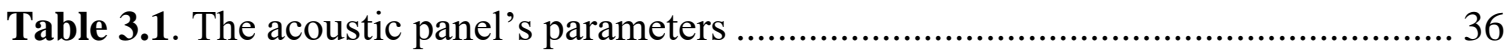

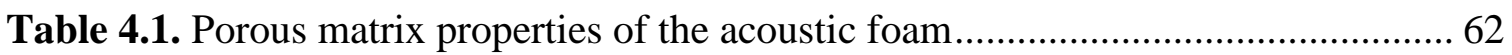

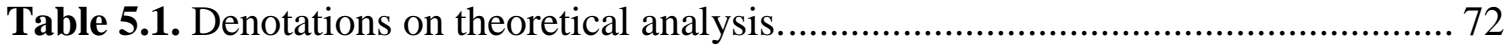

Table 5.2. The dimensions of the individual SHRs. ................................................ 81 


\section{Abstract}

Acoustic metamaterials have been studied intensively recently since they can expose unnatural-born properties, potentially breaking the capacity limits of conventional acoustic materials. Since these interesting properties are mostly observed around metamaterials' local resonances/anti-resonance, resonance-based acoustic metamaterials are most popular in developing metamaterials. Employing resonance-based unnatural born properties such as effective negative mass density, effective negative bulk modulus, and acoustic hyperdamping on designing noise control solutions can give excellent devices showing such high performance that conventional acoustic material cannot achieve. This dissertation is an effort to employ acoustic metamaterials in designing efficient noise control.

First, membrane-type acoustic metamaterials (MAM) will be employed to design a lightweight acoustic panel with high sound transmission loss (STL) in broadband at low frequencies. Negative density at around the anti-resonance of MAM gives it high capability on blocking sound. A double MAM-layer structure is proposed to double the STL performance of unit cells theoretically. Therein, simulation by using COMSOL Multiphysics is the main tool to optimize the unit cell design, panel structure, and effect of panel frame's vibration. Fabrication of the optimal design and experiments are also conducted to verify the calculation and simulation predictions.

In addition to the acoustic panel, MAM is used to design a highly efficient acoustic energy harvester working at low frequencies. A magnet coin is deployed close to a magnet coil attached to the mass of MAM. The maximum oscillation of the coil due to MAM's first local resonance will induce a strong electric current inside the coil. Hence, energy can be 
harvested by an external resistor representing loads of harvesting devices. A complete theoretical model of the harvester is also developed in order to optimize its performance. Multiphysics simulation is conducted to verify the theoretical predictions.

Besides MAM, Helmholtz has been used to design a high-performance and broadband acoustic silencer. Specifically, five slit-type Helmholtz resonators, which possess a massive viscous area, are packed together to create a single-layer silencer. In turn, two single-layer silencers are combined to form a double-layer silencer, which in theory double performance on noise blocking of the single-layer silencer. Theoretical models of slit-type Helmholtz resonators and silencers are developed completely and well validated with simulation and experimental results.

Finally, Fano resonance resulting from the coupling between resonant and non-resonant channels will be explored and employed to design an ultra-broadband acoustic barrier with high ventilation. The resonant channel is generally represented a space-coiling channel, and the non-resonant channel represents ventilation or a straight and short channel. First, the formation of coupling Fano resonance will be theoretically addressed. Subsequently, acoustic hyper-damping is proposed by integrating thin acoustic foams into velocity antinodes in the resonant channel. In the end, an ultra-broadband acoustic barrier with high ventilation and STL is designed by employing three rows of hyper-dampened unit cells. Fabrication and experiment also are conducted to verify the simulation prediction. 


\section{Chapter 1. Introduction}

\subsection{Sound and noise}

Sound is acoustic waves propagating through fluid mediums such as gas, liquid, or solid. In the air medium, air molecules oscillate in a pattern called waves, or sound waves. Regarding the transmitting medium, there are two typical sounds: air-borne and structureborne sounds. While the former refers to the sound transmitted through gases, the latter

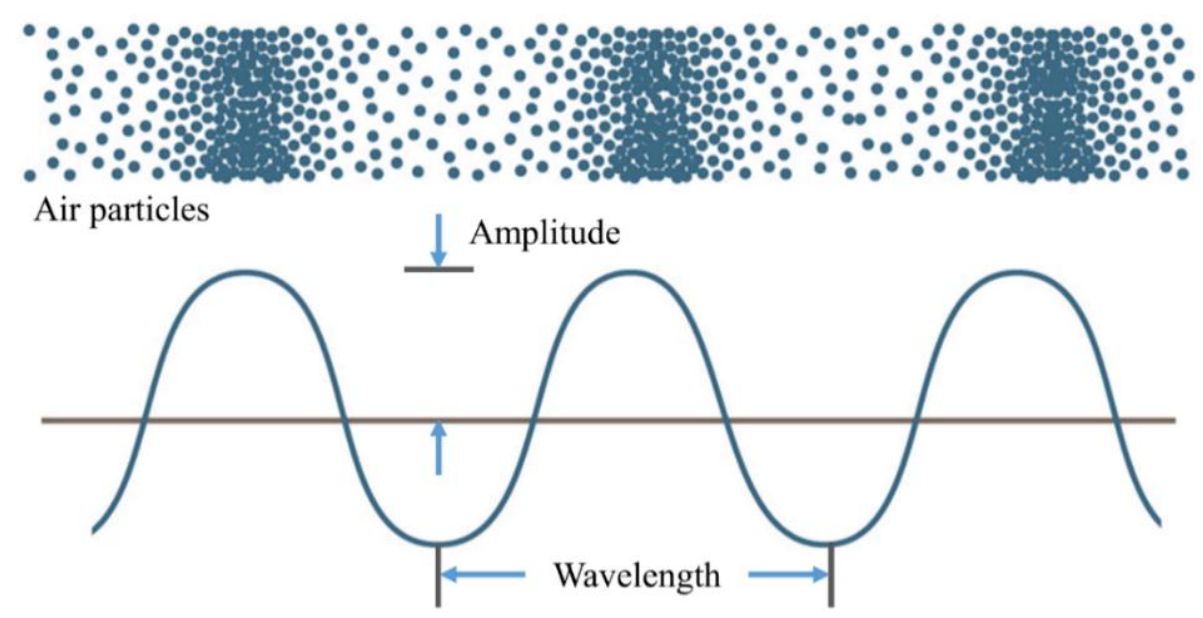

Figure 1.1. The illustration of sound wave propagating through air particles.

mentions the sound propagating in solid structures. Structure-borne sounds are generally resulted from an impact or low-frequency vibration on a solid surface. In terms of frequency, there can be infrasound, audible sound, and ultrasound. The infrasound has frequencies less than $20 \mathrm{~Hz}$, and it usually can be detected by animals such as whales, elephants, giraffes, cats, and alligators. The ultrasound drops on a frequency spectrum higher than $20 \mathrm{kHz}$. It can be detected by bats, dolphins, moths, and lacewings. The audible sound has a frequency spectrum such that the human ear can detect and generally ranges from $20 \mathrm{~Hz}$ to $20 \mathrm{kHz}$. 
The sound wave propagation can be understood by referring to Figure 1.1, which depicts a longitudinal oscillation of air particles. The sparse regions correspond to low pressure and

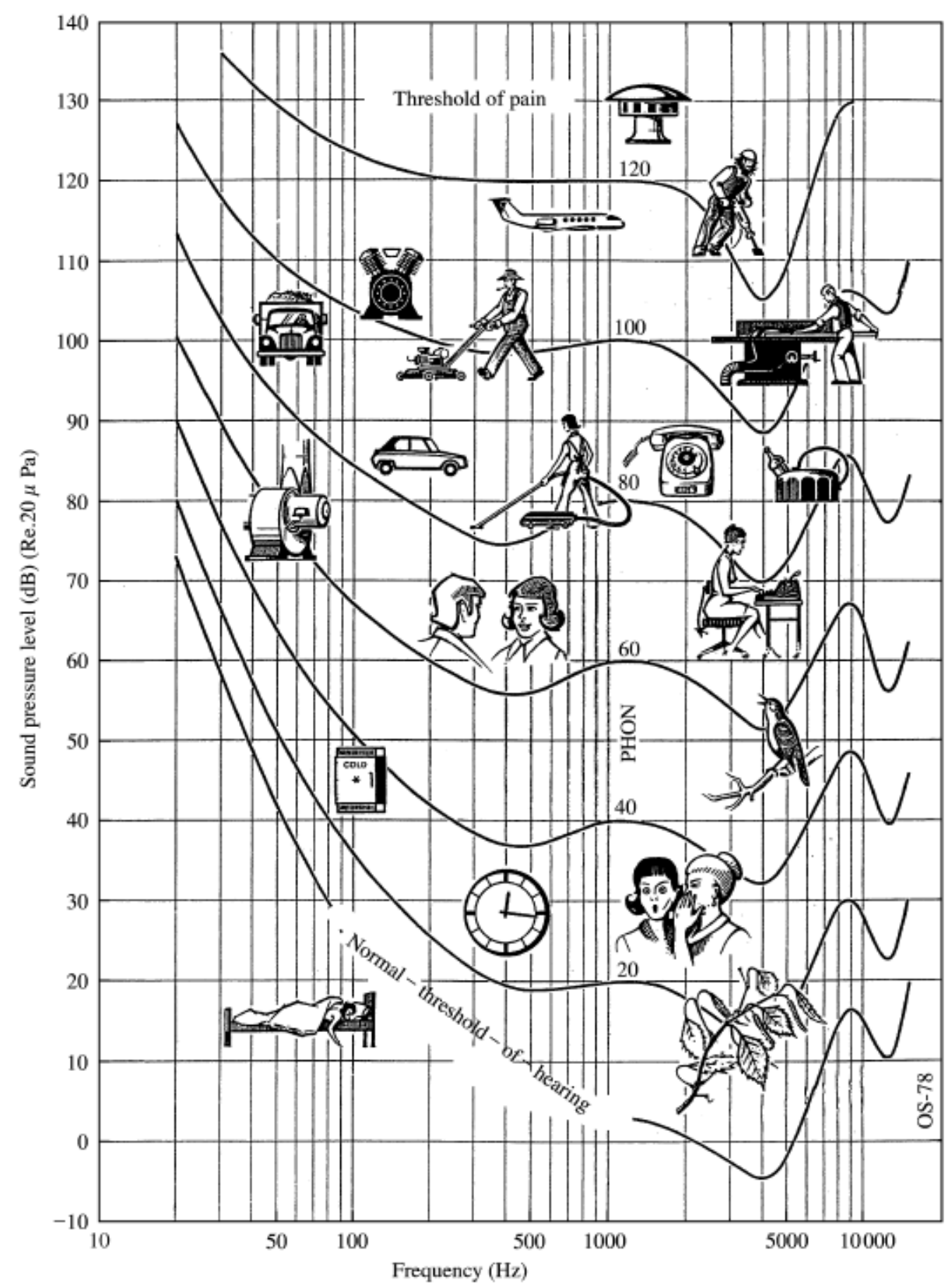

Figure 1.2. The equal loudness contours and the SPL spectrum of some popular noise sources as illustrated by the insets.

hence are called rarefactions, while the dense areas refer to high air pressure and are called compressions. The strength of a sound wave is physically determined from its amplitude, i.e., the higher amplitude, the louder sound. In acoustics, instead of using amplitude 
directly, the sound pressure level defined as SPL $=20 \times \log _{10}\left|\frac{p}{p_{0}}\right|$ having a unit of decibels is employed to evaluate the strength of sound sources.

The strength of sound, which human ears perceive, is called loudness. Mathematically, the higher SPL, the louder sound. However, at different frequencies, human ears have different sensitivity. Generally, human is less sensitive to lower than higher frequency sound. Presented in Figure 1.2 are the equal loudness contours for which a listener perceives the same loudness. The unit of the loudness is phon, and the phon of each contour is the SPL at $1 \mathrm{kHz}$.

Noise is the unwanted sound, which interferes with thinking, concentrating, working, listening, or sleeping. Noise can be generated from highways, traffic roads, airplanes, engine/AC exhaust ventilation, working places, industries, etc. The SPL spectrum of some popular noise sources is presented in Figure 1.2 as well. Noise pollution can cause serious health problems for both people and wildlife and is an invisible danger and one of the main reasons for dissatisfaction with the living environment. It causes serious and long-term harm to human health and working efficiency. One of the main factors leading to dissatisfaction is noise dominated by traffic and industry noise. The traffic noise generally comes from traffic vehicles such as automobiles, buses, trains, airplanes. It is commonly induced by engines, braking, and exhaust, interaction between vehicles and roads, and high-speed air movement. The industry noise generated from industries such as mechanical equipment, generators, refrigeration, beepers, ventilations, and so on has serious effects on residents living close to businesses, industries, and institutional uses. 


\subsection{Noise control}

Noise control is a set of strategies to ease or reduce the impact of noise. There are many approaches used for noise control. However, noise isolation, noise absorption, and noise silencing are the three most popular traditional approaches.

Noise isolation relates to the technique to block noise from transmitting from a region to another region. It often refers to prevent noise from leaking out from its source or transmitting to a specific region. Solutions for noise isolation are generally acoustic panels, separations, and barriers. They can be used for office panels, separation walls, sound fences, and so on. With traditional materials, to block noise efficiently, these products are usually cumbersome.

Noise absorption is a technique used to reduce noise intensity. It can be used in sound absorbers in acoustic rooms to improve the sound quality, lining layers of aircraft engines, ventilation, exhausts, ceilings, or walls to reduce noise refection, etc. Traditional designs prefer using porous materials such as acoustic foams, blankets, and fiberglass. Besides, microperforated plate (MPP) is frequently used for sound absorption applications in harsh environments requiring hard surfaces and exposing to high temperature. The performance of these designs is still mainly affected by the quarter-wavelength principle stating that high-performance designs must have a thickness in wavelength scale. Therefore, they are inefficient for absorbing at low frequencies.

Noise silencing is a technique to absorb and block the exhaust noise and is mostly employed in acoustic silencers and liners. The traditional design of silencers contains several chambers, necks, and perforation separations in order to induce resonances at desired 
frequency ranges. The noise can either be blocked or absorbed at resonant frequencies. However, most of current silencers are winding structures which avoid perfect ventilation and optical transparent.

\subsection{Acoustic metamaterials}

Acoustic metamaterials are structured materials designed to obtain effectively extraordinary acoustic properties, which are limited to traditional materials and generally supported by extreme states of structures. Therefore, most of the acoustic metamaterials expose unnatural-born properties at and around their resonances. These metamaterials hence are sometimes called resonance-based materials. Yang et al. [1] proposed a membrane-type acoustic metamaterial (MAM) comprised of a soft, thin, and pre-stretched membrane with a mass attached at the center. The material can block near-total sound at its anti-resonance that breaks the mass density law deeply. Wherein the effective dynamic mass of the MAM is found negative. Fang et al. [2] observed the negative bulk modulus when studying on a periodic row of Helmholtz resonators. It happens at the resonances of the resonator. Researchers point out that dipolar modes generally support the negative mass while the negative bulk modulus is induced in monopolar modes. Employing both modes in one structure and tuning them to overlap $[3,4]$ the others, a doubly negative acoustic metamaterial can be achieved.

On the one hand, the single negative acoustic metamaterials (negative dynamic mass or negative bulk modulus) have the imaginary part of wavevector negative, which exponentially decays the propagating wave. Therefore, these materials can be employed for sound blocking applications. On the other hand, a double negative metamaterial 
medium allows the wave to propagate throughout it since the wavevector is a purely positive real number. However, the index number of this material is negative, which means the refractive number should be negative accordingly [5]. Therefore, the double negative metamaterials can be used for sound manipulation applications such as superlens for imagining below the diffraction limit, near-field superlens for biomedical imaging, and subwavelength photolithography $[6,7]$.

(a)

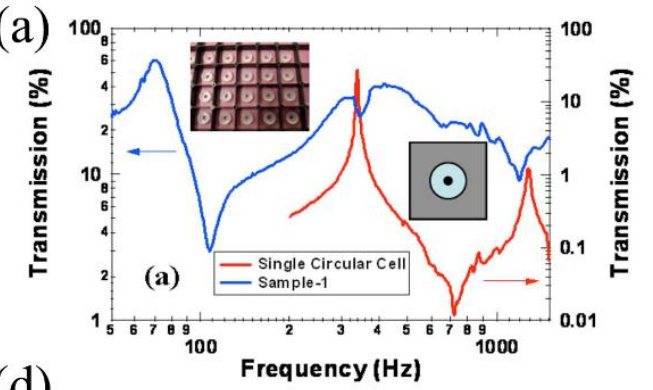

(d)
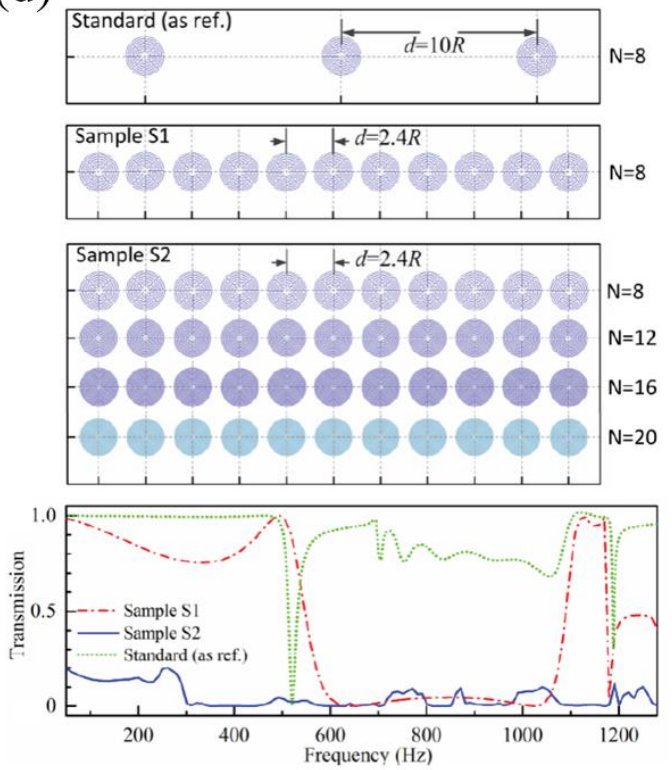

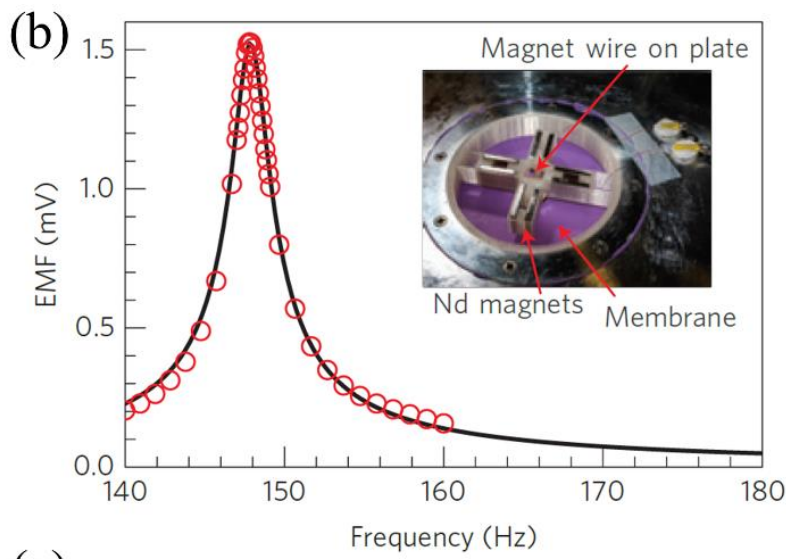

(c)

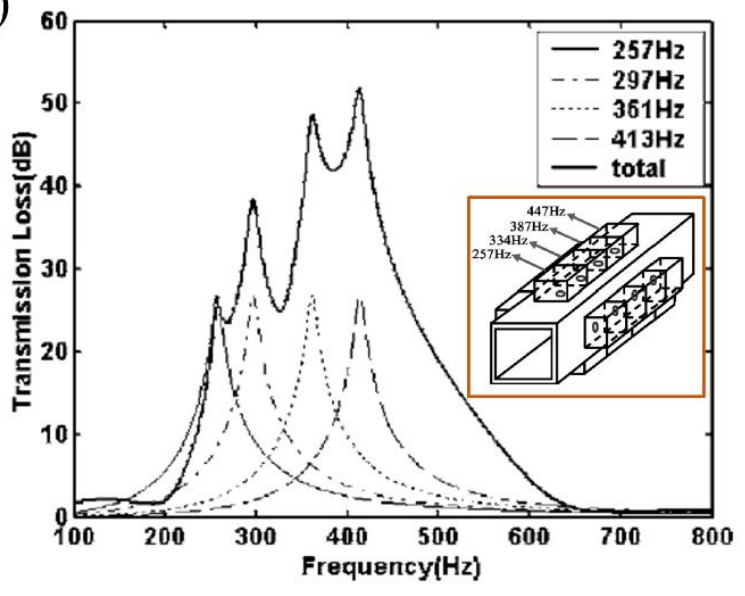

Figure 1.3. (a) The transmission performance of MAM unit cell (red) and single panel (blue) [2]. (b) The electromotive force induced inside the harvesting circuit [4]. (c) The transmission loss performance of individual resonators and silencer [8]. (d) The performance of barriers comprised of Mie resonators [9].

For noise control, acoustic metamaterials have been studied intensively to design deep subwavelength devices compact and working efficiently at designed frequencies. Membrane-type acoustic metamaterials (MAM) have been proposed and showed excellent 
sound transmission loss (STL) and breaking mass density law. The large-scale panels based on MAMs have been proposed to transform the unit cell performance to panel performance $[2,10-12]$. However, the limitation of the proposed design leads to panels' performance lower than predicted on the unit cell scale [see Figure 1.3(a)]. Besides, inspired from metamaterials, local-resonant sonic crystal-based acoustic barrier designs with ventilation have been investigated widely $[8,12,13]$. The induced Fano resonances in these designs, causing either negative mass density or bulk modulus, strongly block the noise. However, the operating frequency bands are not broad enough to satisfy real applications in the industry. In addition to acoustic panels and barriers, low-frequency noise silencers and liners are also interesting acoustic metamaterial applications. MAMs have been combined with MPP to form a new design of acoustic liner, which showed much improvement in absorption at low frequencies [14]. However, its working band is still narrow, and performance in noise blocking is low [see Figure 1.3(d)]. Employing an array of resonators is an alternative way to design acoustic silencers [see Figure 1.3(c)]. The broadband of high STL was observed, but the dimension of the proposed device is large compared to the working wavelength that limits practicing the design in real applications. Finally, efficiency in sound energy harvesting has been significantly improved with metamaterialbased designs. Traditional harvesting designs supported with piezo-electric materials usually give a low harvesting coefficient due to the high impedance of the harvesting materials. Most of the proposed designs have just demonstrated the harvestable qualitatively but quantitatively [15]. Since the fact is that local resonances excite maximum displacement of structures, a MAM-based harvester has been proposed and proved efficiency up to $22 \%$ [see Figure 1.3(b)]. Although the archived results are a significant 
improvement from previous studies, the harvesting efficiency is still small compared to the maximum capacity (100\%) of a harvester. In addition, theoretical analysis on the design, which may be the key to open the path for designing higher efficiency harvesting, has not been investigated yet.

\subsection{Motivations and outline}

In this dissertation, we will employ metamaterial structures on designing noise control devices, which overcome the limitation of the current designs as discussed above. Based on MAMs, a high-efficiency acoustic harvester and a broadband acoustic panel will be proposed. Based on slit-type Helmholtz resonators, broadband and high-performance acoustic silencer will be investigated. And finally, an ultra-broadband acoustic barrier inspired from channel-coupling Fano resonance will be designed.

Specifically, in chapter 2, a MAM-based acoustic harvester is proposed. Magnet damping force, induced at the local resonant mode of MAM, is exploited to convert acoustic energy to electric energy. Complete theoretical model analysis of the harvester is developed and validated with simulation. As a result, the optimal energy conversion of the harvester can reach near its maximum harvesting capacity. In the next chapter, a double MAM-layer unit cell, which has STL performance double single MAM-layer unit cell, is proposed and employed to design a high performance and lightweight acoustic panel. The effect of the frame's vibration on maintaining the performance of unit cells on the panel is also investigated. The experimental result of the final design shows excellent STL in a broadband in middle and low-frequency regions and matches well with simulation. Chapter 4 presents a study on designing a compact and high-performance acoustic silencer based 
on double-layer structure and slit-type Helmholtz resonators. Again, a complete theoretical analysis has been developed and validated well with both simulation and experimental results. In chapter 5, the Fano resonance induced in coupled channels, i.e., a coiling space channel and ventilation channels, first is studied. In turn, thin acoustic foam layers are inserted to optimal positions inside the coiling space channel to excite the acoustic hyperdamping mode of the unit cell. An acoustic barrier based on hyper-dampened unit cells is then designed and optimized to have ultra-broadband noise reduction and absorption. In this dissertation, all theoretical analyses, simulations, and experiments are conducted. The results from these approaches show excellent agreement. Finally, in chapter 5 are some remark conclusions of the dissertation. 


\section{Chapter 2. Membrane-type Harvesting Acoustic Metamaterials}

\subsection{Introduction}

Low-frequency noise (audible sound waves) greatly threatens human health and is a major form of wasted energy. Compared to high-frequency noise, low-frequency noise spreads with modest attenuation through air, is often able to penetrate thick barriers with ease, and is not always easy to control. Based on the conventional mass density law, heavy and massive dissipative materials are needed to decrease low frequency sound transmission; however, lighter weight and compact size are always more desirable characteristics for real-world applications. New tools to control the propagation of these sound waves in the form of new materials are extremely desirable.

Recently, a membrane-type acoustic metamaterial (MAM) comprised of a thin subwavelength-scale microstructure was suggested for low-frequency sound attenuation $[1,3,4,16,17]$. This type of acoustic metamaterial has attracted large interest in the research community because it has a relatively simple geometry combined with an intriguing capability to dissipate low-frequency sound waves based on the local resonant mechanism. Based on the MAM design, the lightweight simple structures $[18,19]$ which expose broadband attenuation, and pure flexible design [20] have been proposed. Compared with conventional sound attenuation materials typically utilizing thermally coupled dissipation mechanisms and suffering from inadequate low frequency sound attenuation, MAMs can be designed to possess nearly total reflection and/or absorption for targeting low-frequency acoustic sources. 
The basic structure of this MAM consists of a prestressed membrane with one or multiple attached, small, heterogeneous masses acting as resonators. The unusual low-frequency vibroacoustic behavior has been completely characterized, and the response spectrum shows separate transmission and absorption peaks around resonances [21, 22]. The lowfrequency sound transmission mechanism has also been numerically explained through effective mass density and averaged normal displacement by using the finite element method, although the nearly total reflection and absorption of the MAM is of limited frequency bandwidth.

As one of many potential engineering applications, lightweight MAM honeycomb panels have been designed numerically and experimentally to mitigate low frequency noise specifically in aerospace structures [23]. For passive acoustic metamaterials, the operative wave frequencies are hardly adjustable once fabricated. Thus, they cannot adapt to real-life scenarios under ambient environments. One promising way to mitigate these problems is to incorporate an active element. Chen et al. used a gradient magnetic field to actively tune the membrane-type acoustic metamaterials (MAMs) [24]. Xiao et al. also investigated acoustic properties of membrane-type metamaterials actively adopted by external voltage to illustrate phase modulation and acoustic wave switch [25].

In another vein, scientists and engineers have been strongly interested in potential applications of acoustic metamaterials in the field of energy harvesting. The unique ability of acoustic metamaterials to trap acoustic waves in a localized regime can be used to harvest sound energy efficiently through integrating smart elements and electric circuits. From a practical point of view, there is combined value in both protecting people from damaging sound as well as actively retrieving energy from these noises to provide power 
to electronic devices without the need of batteries. As one of several candidate materials, piezoelectric materials have been successfully used in acoustic metamaterials for harvesting structure-borne as well as air-borne sound energies [26-31]. Although piezoelectric materials can generate a relatively high-voltage output from external motioninduced mechanical strain, the high impedance in piezoelectric-based harvesters mandates the load impedance to be high, which will significantly limit the acoustic energy harvesting efficiency [32-34]. Beside employing piezoelectric materials on designing harvesting structures, the voltage-tunable [35] and dielectric elastomer based [36] designs are also taken a lot of attention. Although these designs show very good ability on acoustic absorption, the harvested energy and ability on harvesting acoustic energy have not mentioned yet.

An alternative way to design an energy harvester is based on electromagnetic energy conversion, which is suitable for a low impedance and therefore can generate a high output current [37]. Mikoshiba et al. [38] designed an acoustic metamaterial consisting of a springloaded magnet enclosed in a capped poly(methyl methacrylate) tube equipped with copper coils to harvest vibrational energy using electromagnetic induction. Ma et al. [39] demonstrated a membrane acoustic metasurface that can convert the acoustic energy to electric current through electromagnetic induction with a power conversion efficiency of $23 \%$, which demonstrates great potential for acoustic energy harvesting. However, many design issues have yet to be resolved and better understood such as enhancing efficiency, optimizing microstructures, and operating at a frequency bandwidth. A solid analytical model that can accurately capture the complicated vibro-acoustic-electromagnetic coupling behavior is greatly needed. The analytical method can provide both computational 
efficiency and flexibility, and therefore can be very useful in the design of multi-functional MAMs for desired engineering applications.

In this dissertation, we developed a comprehensive vibro-acoustic-electromagnetic coupling model to capture the dynamic behavior as well as the energy absorption, conversion and harvesting in the proposed MAM harvester accurately. Based on the model, sound absorption, acoustic-electronic energy conversion, and the MAM harvesting coefficient are quantitatively evaluated for various geometries and constitutive material properties of the metamaterial. The efficiencies of energy conversion and energy harvesting, the maxima of which are $48 \%$ and $36 \%$, respectively, are analyzed and optimized by varying the subwavelength-scale microstructure and connected circuit configurations. Numerical simulations are conducted to validate the analytical solutions, and excellent agreement is observed.

\subsection{Analytical modeling of the Magneto-based Acoustic Metamaterial} Harvester

Without loss of generality, the magneto-based acoustic metamaterial harvester is considered as a MAM with a circular mass attached at the center of the membrane, a torusshape magnet wire attached to the mass, and a permanent magnet placed close to the magnet wire, as shown in Figure 2.1(a). When the incident pressure wave excites the membrane, the mass and attached wire vibrate in the magnetic field generated by the permanent magnet, which will induce a current in the moving wire. Thus, an external magnetic force acting on the mass and wire is initiated to prevent the vibration of the mass and magnet wire accordingly. By connecting a resistor, $R_{l}$, into the circuit connected to the 
wire, the absorbed acoustic energy will be dissipated partially through the resistor $R_{l}$. This energy is called harvested energy. Note that the magnet coil also has an internal load or internal resistance denoted by $R_{i}$. And the electric energy dissipated through $R_{i}$ always becomes heat, therefore, can be called wasted energy.

(a)

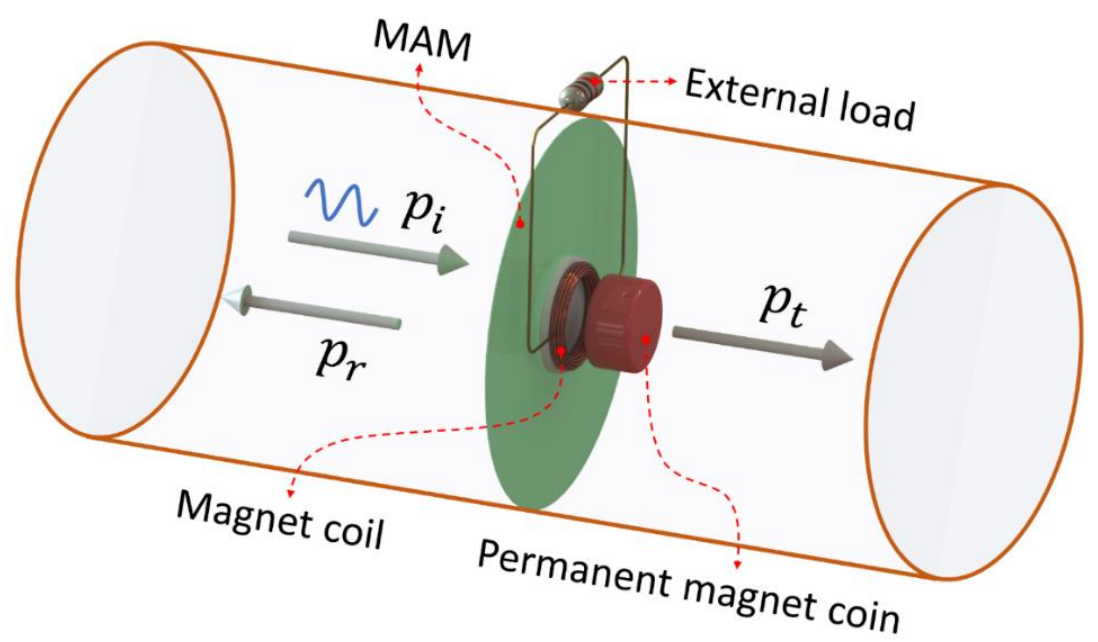

(b)

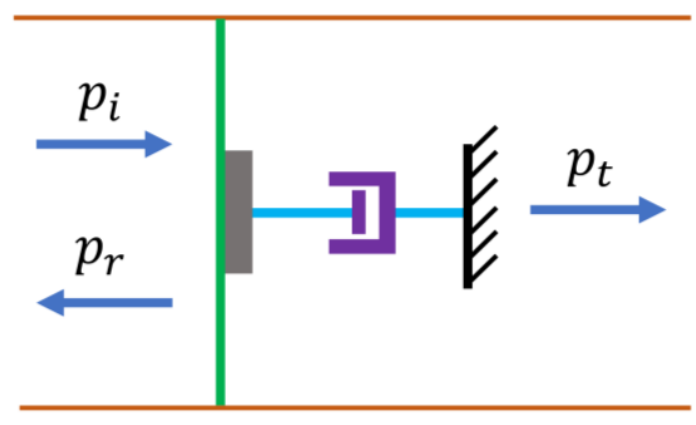

(c)

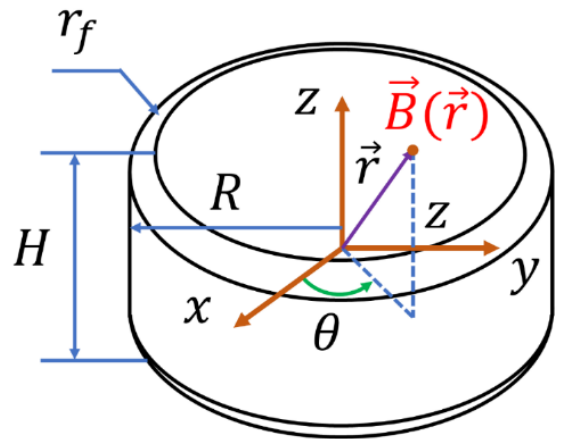

Figure 2.1. (a) - the model of the MAM harvester, (b) - the mechanical equivalent of the electromagnetism part of the MAM harvester, (c) - the model of the permanent magnet coin.

In this dissertation, we focus on characterizing the MAM sound absorption in the tube subject to a plane normal sound wave and investigate the energy harvesting ability of the magneto-based acoustic metamaterial harvester. Perfectly absorbing boundary conditions are assumed in both ends of the tube so that there are no multiple reflected waves to the MAM. First, the external magnetic force is obtained analytically by considering the 
interaction between the magnetic field and the moving electric circuit. Then, the vibroacoustic-magnetic coupling behavior of the MAM is analyzed through the modal superposition theory, from which the sound transmission and reflection of the MAM can be analytically determined. Finally, the energy harvesting ability and its efficiency are quantitatively estimated by connecting electric circuits to the wire. This model can also be easily extended to analyze the MAM-based harvesters with multiple attached masses in arbitrary shapes.

\subsubsection{The determination of magnetic force}

For a permanent magnet coin, as shown in Figure 2.1(c), the magnetic field around the magnet can be described as [40]

$$
\vec{B}(\vec{r})=\frac{\mu_{0}}{4 \pi}\left\{\int_{V} \frac{(\nabla \times \vec{M}) \times\left(\vec{r}-\overrightarrow{r_{V}}\right)}{\left|\vec{r}-\vec{r}_{V}\right|^{3}} d V+\int_{S} \frac{(\vec{M} \times \vec{n}) \times\left(\vec{r}-\overrightarrow{r_{S}}\right)}{\left|\vec{r}-\vec{r}_{S}\right|^{3}} d S\right\}
$$

where, the magnetization $\vec{M}$ is constant in the $\vec{Z}$ direction. Since the harvester is axisymmetric, only the magnetic field in the radial direction affects the motion of the harvester. Therefore, we rewrite $B_{r}$ as

$$
\begin{aligned}
& B_{r}(r, z)=\frac{\mu_{0}}{4 \pi} M \int_{0}^{2 \pi} \int_{-\frac{H}{2}}^{-\left(\frac{H}{2}-r_{f}\right)} \frac{\left(z-z_{S}\right) \cos \varphi_{S}}{\left|r^{2}+r_{S}^{2}-2 r r_{S} \cos \varphi_{S}+\left(\mathrm{z}-z_{S}\right)^{2}\right|^{3 / 2}}\left[R-r_{f}(1-\cos \theta)\right] d \varphi_{S} d z_{s}+ \\
& \frac{\mu_{0}}{4 \pi} M \int_{0}^{2 \pi} \int_{-\left(\frac{H}{2}-r_{f}\right)}^{\left(\frac{H}{2}-r_{f}\right)} \frac{\left(z-z_{S}\right) \cos \varphi_{S}}{\left|r^{2}+r_{S}^{2}-2 r r_{S} \cos \varphi_{S}+\left(z-z_{S}\right)^{2}\right|^{3 / 2}} R d \varphi_{S} d z_{S} \\
& +\frac{\mu_{0}}{4 \pi} M \int_{0}^{2 \pi} \int_{\left(\frac{H}{2}-r_{f}\right)}^{\frac{H}{2}} \frac{\left(z-z_{S}\right) \cos \varphi_{S}}{\left|r^{2}+r_{S}^{2}-2 r r_{S} \cos \varphi_{S}+\left(z-z_{S}\right)^{2}\right|^{3 / 2}}\left[R-r_{f}(1-\cos \theta)\right] d \varphi_{S} d z_{S}
\end{aligned}
$$


where, $\mu_{0}=4 \pi \times 10^{-7} \mathrm{~N} / \mathrm{A}^{2}$ is the permeability of the free space; and $\sin \theta=\frac{\left[z_{s}+\left(\frac{H}{2}-r_{f}\right)\right]}{r_{f}}$; $R, H, r_{f}$, and $M$ are the radius, height, fillet radius, and magnetization of the permanent magnet, respectively.

When the mass and attached wire move back and forth inside the magnetic field $\vec{B}$ with velocity $\vec{v}$, the electromotive force (EMF) induced inside the circuit reads

$$
\epsilon=-\frac{d \phi}{d t}=\oint_{L}(\vec{v} \times \vec{B}) \cdot d \vec{l} \approx N \oint_{\text {loop }}(\vec{v} \times \vec{B}) \cdot \vec{s} d l
$$

where $\vec{S}$ and $N$ are the tangential vector and the number of the loops of the wire, respectively.

The current density induced inside the connected circuit is then

$$
j=\frac{\epsilon}{A\left(R_{i}+R_{l}\right)}
$$

where the external resistor is denoted as $R_{l}$ and the internal resistance of the magnet coil is $R_{i}=\frac{4 N^{2} d_{o}}{\sigma_{c} d_{c}^{2}}$ with the number of the loops of the wire being $N \approx\left(\frac{d_{c}}{d_{w}}\right)^{2}$ and $d_{o}, d_{w}, d_{c}$ and $\sigma_{c}$ are the diameters of the coil, magnet wire, the coil's cross-section and the electrical conductivity of the magnet wire, respectively.

Due to axisymmetric harvesting, the external distributed force acting on the wire can then be determined as

$$
\overrightarrow{f_{b}}=\vec{\jmath} \times \vec{B}=-\frac{\pi d_{o} N B_{r}^{2} \dot{w}_{m}}{A\left(R_{i}+R_{l}\right)} \vec{n}_{z}
$$


herein, the radial component of the magnetic field is ignored since it will be canceled out in summation; $d_{c}, A, \vec{v}$ and $\dot{w}_{m}$ are the diameter of the wire, cross-sectional area of the wire, the velocity vector of the mass and its z-directional component $\left(\dot{w}_{m}=v_{z}\right)$, respectively. Thus, the total induced magnetic force can be determined by summing the distributed forces along the wire as

$$
\vec{F}_{m}=\int_{\text {coil }} \vec{f}_{b} d l=-\frac{\left(\pi d_{o} N B_{r}\right)^{2}}{R_{i}+R_{l}} \dot{w}_{m} \vec{n}_{z}
$$

Since the magnetic force is proportional to the velocity of the mass, physically the magnetic force acts exactly like a damping force to the membrane with the magnet damping coefficient being $\beta_{m}=\frac{\left(\pi d_{o} N B_{r}\right)^{2}}{R_{i}+R_{l}}$, as shown in the Figure 2.1(b). In the frequency domain, the average power absorbed by the magnetic damper, therefore, can be calculated as

$$
W_{d}=\frac{\omega}{2 \pi} \int_{0}^{2 \pi / \omega} \mathcal{R}_{e}\left\{F_{m}\right\} \cdot \mathcal{R}_{e}\left\{\dot{w}_{m}\right\} d t=\frac{1}{2} \frac{\left(\pi d_{o} N B_{r}\right)^{2}}{R_{i}+R_{l}}\left|\dot{w}_{m}\right|^{2}
$$

Note that, for the system without other energy dissipation, the electromagnetic part of the harvester should be equivalent to a mechanical damper that is proportional to the damping coefficient $\beta_{m}$.

By further analyzing the damping coefficient in the form of $\beta_{m}=\frac{\left(\pi d_{o} B_{r}\right)^{2}}{4 d_{o} /\left(\sigma_{c} d_{c}^{2}\right)+R_{l} / N^{2}}$, we can conclude that the conversion coefficient depends on the ring diameter and ring crosssectional diameter, and reaches its maximum value when zero load is applied. 


\subsubsection{Coupling Vibro-Acoustic-Electromagnetic model of the MAM}

Since the attached mass and wire in the MAM are assumed to be bonded perfectly to the membrane and rigid compared with the deformable membrane, to properly capture effects of the finite mass on the small deformation of the membrane, the proposed MAM is decomposed into two parts: an annulus membrane and a mass with the attached magnet coil. Considering the elastic membrane here as an elastic plate, the governing equation of the annulus pre-tension membrane can be written as $[22,41]$

$$
\bar{D} \nabla^{4} w-T \nabla^{2} w-\rho h \omega^{2} w=0
$$

with the boundary conditions (BCs)

$$
\begin{array}{lll}
w=\frac{\partial w}{\partial r}=0, & \text { at } & r=a \\
\frac{\partial w}{\partial r}=0,2 \pi \bar{V}(b)=m \ddot{w}(b) & \text { at } & r=b
\end{array}
$$

where $\bar{D}=\frac{h^{3}}{12}\left(\sigma_{0}+\frac{E}{1-v^{2}}\right)$ is the effective bending stiffness of the membrane, $T=\sigma h$ is the pretension in the membrane, and $\bar{V}(b)$ is the shear force at radius $b$ [41]

$$
\bar{V}(b)=V(b)+\left.T \frac{\partial w}{\partial r}\right|_{r=b}
$$

where $V(b), h$ and $\rho$ are the Kirchhoff's shear force at the inner boundary, the thickness, and mass density of the annulus membrane, respectively.

The general solution of the equation (2.8) can be expressed as [41, 42]

$$
w(r)=A J_{0}\left(\gamma_{1} r\right)+B Y_{0}\left(\gamma_{1} r\right)+C I_{0}\left(\gamma_{2} r\right)+D K_{0}\left(\gamma_{2} r\right)
$$


where

$$
\gamma_{1,2}=\sqrt{\sqrt{\alpha^{4}+\beta^{4}} \mp \alpha^{2}} \quad \alpha^{2}=\frac{T}{2 D} \quad \beta^{4}=\frac{\rho h \omega^{2}}{\bar{D}}
$$

$J_{0}, Y_{0}$ and $I_{0}, K_{0}$ are the zero-order Bessel functions and modified Bessel functions, respectively.

Since only the steady-state response field will be considered, the time factor $e^{i \omega t}$, which applies to all the field variables, will be suppressed.

By using the BCs, the natural frequencies and mode shape functions of the MAM can be determined by solving the eigen-value problem of the matrix

$$
\begin{array}{|cccc|}
J_{0}\left(\gamma_{1} a\right) & Y_{0}\left(\gamma_{1} a\right) & I_{0}\left(\gamma_{2} a\right) & K_{0}\left(\gamma_{2} a\right) \\
\gamma_{1} J_{1}\left(\gamma_{1} a\right) & \gamma_{1} Y_{1}\left(\gamma_{1} a\right) & -\gamma_{2} I_{1}\left(\gamma_{2} a\right) & \gamma_{2} K_{1}\left(\gamma_{2} a\right) \\
\gamma_{1} J_{1}\left(\gamma_{1} b\right) & \gamma_{1} Y_{1}\left(\gamma_{1} b\right) & -\gamma_{2} I_{1}\left(\gamma_{2} b\right) & \gamma_{2} K_{1}\left(\gamma_{2} b\right) \\
\gamma_{1}^{3} J_{1}\left(\gamma_{1} b\right)-\alpha J_{0}\left(\gamma_{1} b\right) & \gamma_{1}^{3} Y_{1}\left(\gamma_{1} b\right)-\alpha Y_{0}\left(\gamma_{1} b\right) & \gamma_{1}^{3} I_{1}\left(\gamma_{2} b\right)-\alpha I_{0}\left(\gamma_{2} b\right) & -\gamma_{2}^{3} K_{1}\left(\gamma_{2} b\right)-\alpha K_{0}\left(\gamma_{2} b\right)
\end{array} \mid
$$

Next, consider a plane sound wave is normally incident on the MAM. Per the fact that the thickness of the MAM is extremely small compared with the wavelength of low-frequency sound in air, thickness effects of the MAM can be ignored. The objective is to determine the acoustic energy reflection, transmission, and absorption within the MAM. The governing equation of the acoustic excited membrane based on the plate theory and motion equations of the mass can be expressed as

$$
\begin{aligned}
& \bar{D}(1+i \omega \eta) \nabla^{4} w-T \nabla^{2} w-\rho \omega^{2} w=\Delta p+\bar{V} \delta(r-b) \\
& -2 \pi b \bar{V}+F_{m}+\int_{S_{i}} \Delta p d S=-m \omega^{2} w(b)
\end{aligned}
$$


where $S_{i}$ denotes the circular surface of the mass, and $\Delta p$ is the total pressure acting on the membrane

$$
\Delta p=\left.\left(p_{i}+p_{r}-p_{t}\right)\right|_{z=0}=2\left[P_{i}-i \omega\langle w\rangle+\omega k_{a} Z_{0} \int_{S} \delta G\left(r \mid r^{\prime}\right) w\left(r^{\prime}\right) d r^{\prime}\right]
$$

where $S$ is the surface of the MAM; $Z_{0}=\rho_{0} c_{0}$ is the specific characteristic impedance of air; $P_{i}$ is the amplitude of the incident pressure wave; $\langle w\rangle$ is the average displacement of the membrane; and $\delta G\left(r \mid r^{\prime}\right)$ is the deviation of the acoustic Green's function of the tube measured at $z=0[43]$

$$
\delta G\left(r \mid r^{\prime}\right)=\frac{1}{\pi a^{2}} \sum_{l=1}^{\infty} \frac{J_{0}\left(k_{r l} r\right) J_{0}\left(k_{r l} r^{\prime}\right)}{J_{0}^{2}\left(k_{r l} a\right) \sqrt{k_{r l}^{2}-k_{a}^{2}}}
$$

where $k_{a}$ is the wavenumber of air and $k_{r l}$ is the $l^{\text {th }}$ order wavenumber of air in the radial direction of the waveguide.

The dissipative property of the membrane is employed here through the imaginary part of the Young's modulus, viscosity $\chi$, and effective loss factor $\eta$ of the membrane as

$$
E_{m}=E+i \omega \chi \quad \eta=\frac{\chi}{E+\left(1-v^{2}\right) \sigma_{0}}
$$

where $E$ and $\sigma_{0}$ are the Young's modulus and pre-tension of the membrane.

The superposition method is adopted by assuming the solution in the form

$$
w(r)=\sum_{k} \lambda_{k} w_{k}(r)
$$

where $w_{k}(r)$ is the $k^{t h}$ eigen function of the annulus membrane. 
Multiplying $w_{n}$ in equation (2.15) and conducting an integral over the surface of the annulus membrane $S_{o}$ lead to

$$
\left.\begin{array}{rl}
\sum_{k} \lambda_{k}\left\{i \omega \eta T \int_{S_{o}} w_{n} \nabla^{2} w_{k} d S_{o}\right. \\
+\delta_{n, k}\left(\omega_{k}^{2}+i \omega_{k}^{2} \eta \omega-\omega^{2}\right)\left[\rho h \int_{S_{o}} w_{n}^{2} d S_{o}+m w_{n}^{2}(b)\right] \\
+i \omega \beta_{m} w_{n}(b) w_{k}(b)+2 i \pi a^{2} \omega Z_{0}\left\langle w_{n}\right\rangle\left\langle w_{k}\right\rangle \\
-\frac{2 i \omega k_{a} Z_{0}}{\pi a^{2}} \sum_{l=1}^{\infty} \frac{\int_{S} J_{0}\left(k_{r l} r\right) w_{n}(r) d r \int_{S} J_{0}\left(k_{r l} r^{\prime}\right) w_{k}\left(r^{\prime}\right) d r^{\prime}}{J_{0}^{2}\left(k_{r l} a\right) \sqrt{k_{r l}^{2}-k_{a}^{2}}} \\
=2 \pi a^{2} P_{i}\left\langle w_{n}\right\rangle
\end{array}\right\}
$$

The equation (2.21) is recognizable as a system of linear equations where unknown variables $\lambda_{k}$ can be numerically determined.

Therefore, the far field transmission, reflection coefficients and absorption for radiated plane waves of the MAM can be expressed as

$$
T=\frac{i \omega Z_{0}}{P_{i}} \sum_{k} \lambda_{k}\left\langle w_{k}\right\rangle, \quad R=1-T \text { and } \quad A=1-|R|^{2}-|T|^{2}
$$

respectively.

For the acoustic energy characterization, the acoustic input energy is calculated as

$$
W_{i n}=\frac{\pi a^{2}\left|P_{i}\right|^{2}}{2 Z_{0}}
$$


and the energy absorbed by the elastic membrane, or elastic absorption (due to deformable elastic dissipation), which can be determined by subtracting the energy absorbed by the magnetic damper and the elastic absorption coefficient, are presented as

$$
W_{m}=A \cdot W_{i n}-W_{d} \text { and } A_{m}=\frac{W_{m}}{W_{i n}}=A-\frac{W_{d}}{W_{i n}},
$$

respectively. On the other side, the harvested energy through connecting the external resistor $R_{l}$ is

$$
W_{h}=\frac{R_{l}}{R_{i}+R_{l}} W_{d}
$$

and the acoustic energy conversion and harvesting coefficients of the MAM harvester can be expressed as

$$
c=\frac{W_{d}}{W_{\text {in }}} \text { and } \varphi=\frac{W_{h}}{W_{\text {in }}}=\frac{R_{l}}{R_{i}+R_{l}} c
$$

\subsection{Results and discussion}

To verify the theoretical model of the MAM harvester, several configurations of theoretically derived acoustic absorption analyses are compared with simulation results derived from the commercial code, COMSOL Multiphysics. Since the magnetic field generated from the permanent magnet is stationary, a two-step study is employed in the numerical simulation. In the first step, a 'Stationary' study on the 'Magnetic Fields, No Currents (MFNC)' module is selected to obtain the static permanent magnetic field. In the second step, a 'Frequency Domain' on the 'Acoustic-Structure Interaction' module is performed to study the interaction between acoustic domains and MAM. The magnetic 
effect is considered by applying a velocity-dependent body force on the magnet coil calculated from the magnetic field and the mass' velocity. The MAM parameters used in analytical modeling and numerical simulation are listed in the Table 2.1. The diameter of the magnet wire is set to $0.104 \mathrm{~mm}$, and the internal resistance of the magnet coil $R_{i}$, therefore, is equal to $0.55 \Omega$.

Table 2.1. The MAM harvester's parameters.

\begin{tabular}{|c|c|c|c|}
\hline Element & Property & Symbol & Value \\
\hline \multirow{7}{*}{ Elastic membrane } & Radius & $a$ & $5 \mathrm{~mm}$ \\
\hline & Thickness & $h$ & $0.1 \mathrm{~mm}$ \\
\hline & Young's modulus & $E$ & $1.9 \times 10^{6} \mathrm{~Pa}$ \\
\hline & Poisson's ratio & $v$ & 0.48 \\
\hline & Mass density & $\rho$ & $980 \mathrm{~kg} / \mathrm{m}^{3}$ \\
\hline & Prestress & $\sigma_{0}$ & $2.2 \times 10^{5} \mathrm{~Pa}$ \\
\hline & Viscosity & $\chi$ & $796 \mathrm{~Pa} \cdot \mathrm{s}$ \\
\hline \multirow{3}{*}{ Attached mass } & Radius & $b$ & $5 \mathrm{~mm}$ \\
\hline & Thickness & $t$ & $0.35 \mathrm{~mm}$ \\
\hline & Mass density & $\rho_{m}$ & $2900 \mathrm{~kg} / \mathrm{m}^{3}$ \\
\hline \multirow{4}{*}{$\begin{array}{l}\text { Permanent magnet } \\
\text { coin }\end{array}$} & Radius & $R$ & $3 \mathrm{~mm}$ \\
\hline & Height & $H$ & $3 \mathrm{~mm}$ \\
\hline & Fillet radius & $r_{f}$ & $0.375 \mathrm{~mm}$ \\
\hline & Magnetization & $M$ & 750 kA/m \\
\hline Magnet coil & Diameter of the coil & $d_{o}$ & $6 \mathrm{~mm}$ \\
\hline
\end{tabular}




Diameter of the magnet
coil's cross-section $\quad d_{c} \quad 0.4 \mathrm{~mm}$

The gap in between the mass and the permanent

$\Delta$

$0.5 \mathrm{~mm}$ magnet

Electrical conductivity

$\sigma_{c}$

$6 \times 10^{7} \mathrm{~S} / \mathrm{m}$

Mass density

$\rho_{w}$

$8960 \mathrm{~kg} / \mathrm{m}^{3}$

To validate the theoretical model, the two major results predicted by the theoretical analysis and numerical simulation are compared. First, we compare the magnetic fields measured at the center of the magnet coil's cross-section $B_{r}$ by employing equation (2.2) versus COMSOL methods. The results for $B_{r}$ are $-0.29278 T$ and $-0.29267 T$ respectively, which shows the tremendous accuracy of the theoretical magnet calculation. Second, we compare the wave absorption of the MAM harvester predicted from the theoretical analysis versus the numerical simulation based on the commercial code COMSOL Multiphysics as shown in the Figure 2.2. Three cases of the harvester are: (1) viscous membrane (elastic membrane with dissipative property) but without magnet effect; (2) non-viscous membrane with magnet effect; and (3) both viscous membrane and the magnet effect. The results from the theoretical analysis and the simulation are nearly perfectly matched. The developed theoretical analysis therefore is highly precise and correlates extremely well with numerical simulation. Furthermore, while the dissipated energy from the viscous membrane is very small (less than 5\%), the absorption of the harvester under the magnet effect is significantly improved (34\%). The proposed harvester configuration thus demonstrates a very high potential to harvest energy. Next, the theoretical analysis is employed to analyze the parameter effect and further optimize the harvester. 


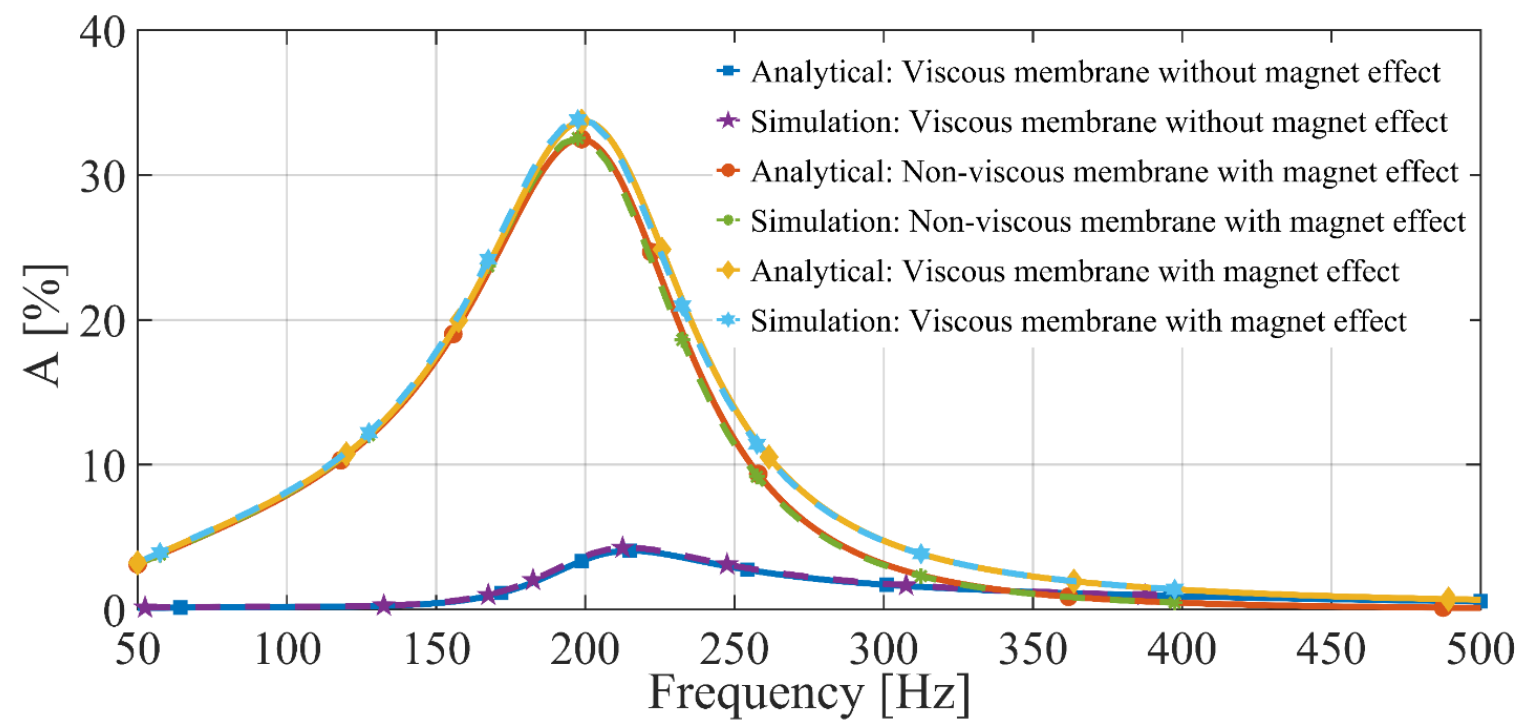

Figure 2.2. The comparison between the simulation and analytical results for the cases the membrane with dissipative property but without effect of the magnet, the membrane without dissipative property but with the magnet effect, the membrane with both dissipative property and the magnet effect.

Figure 2.3(a) and (b) show the effect of the external load $R_{l}$ on the conversion and harvesting coefficients of the MAM harvester. In the figures, the material parameters of the MAM are the same as those listed in Table 2.1. Through analysis we have shown that the conversion coefficient, which corresponds to the amount of energy absorbed by the circuit, decreases when the external load $R_{l}$ increases. The best conversion coefficient is observed when the external load is zero. This phenomenon is explained by considering the effect of the magnet damping coefficient $\beta_{m}$ : an increased external load $R_{l}$ lowers the magnet damping coefficient, which reduces the dissipated energy through the circuit.

The figures also shows that all the conversion coefficient peaks are located in the hybridized resonant frequency [39], $198 \mathrm{~Hz}$. Due to small elastic dissipative energy (elastic absorption), the conversion coefficient peaks, and the absorption of the harvester are at very close frequencies. The conversion peak here matches with the first absorption peak at the hybridized resonant frequency, which is very close to the first anti-resonant frequency. The first anti-resonance, on the other hand, occurs when the real part of the acoustic 
Green's function of the MAM [39] is equal to zero and completely depends on the first two resonant frequencies. The dissipative property of the membrane and the magnet damping coefficient are only related to the resistance of the harvester and will not contribute to the resonant frequencies, which is also confirmed from the absence of these factors in the characteristic equation (2.18). Therefore, the peak conversion coefficient in the hybridized resonant frequency will not change when the external loads are varied. The harvesting coefficient $\varphi$ of the harvester presented in Figure 2.3(b), which is defined as the amount of the energy dissipated through the external load $R_{l}$, on the other hand, does not follow the trend of the conversion coefficient. It will initially increase, then reach the best performance when $R_{l} / R_{i} \approx 1.25$, and afterward decrease with the increase the external load. The harvested energy depends not only on the ratio of external load to internal load $\left(R_{l} / R_{i}\right)$ in equation (2.25), but also on the amount of energy converted to electric energy. The increase of $R_{l}$ will lower the magnet damping coefficient, and therefore make the conversion energy decrease. Therefore, the increase of the external load $R_{l}$ does not ensure the harvested energy is increased as observed in Figure 2.3(b). In addition, the harvested energy is proportional to the conversion energy [see equation (2.26)], and the peak of the harvesting coefficient is also located at the hybridized resonant frequency.
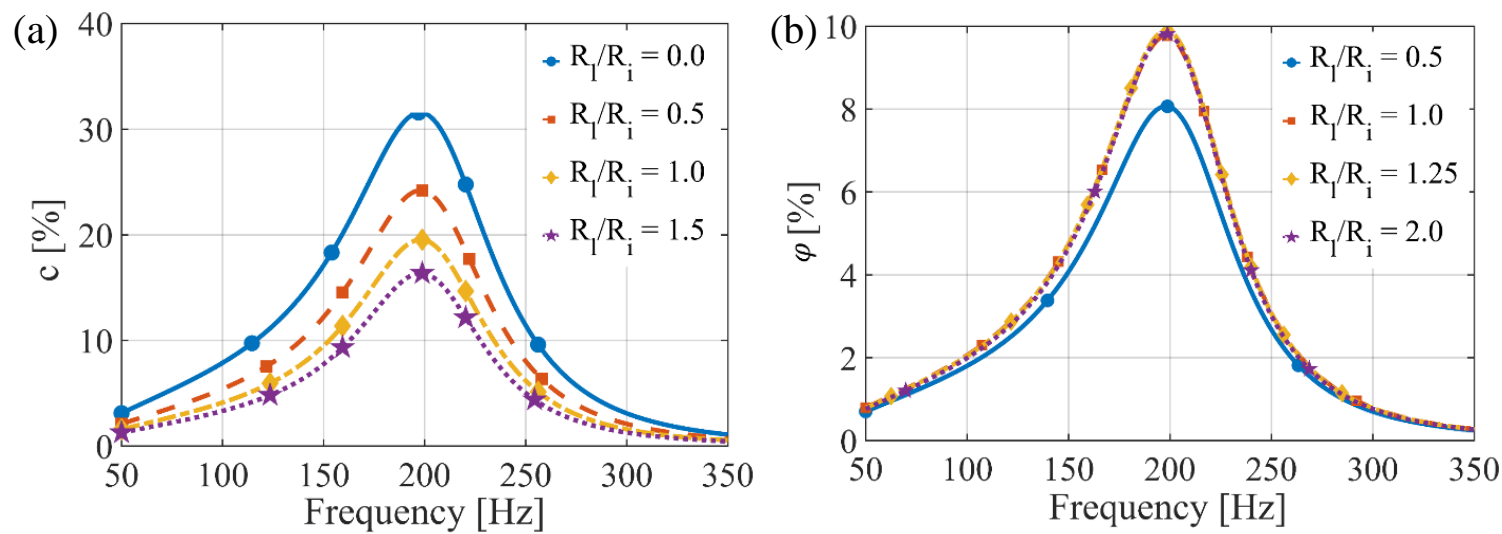

Figure 2.3. The effect of the external load on the conversion (a) and harvesting (b) coefficients. 
The next geometric parameter studied is the thickness of the membrane $h$. To get a higher conversion coefficient, the external load $R_{l}$ is set at zero, and other parameters are the same as listed in Table 2.1. When the membrane becomes thicker (or $h$ increases), the effective bending stiffness of the membrane increases, which theoretically makes the resonant frequencies of the harvester also increase. Consequently, the peaks of the conversion coefficient and the elastic dissipative energy through the membrane shift to higher frequencies as observed in Figure 2.4(a) and Figure 2.4(b). When the membrane becomes thicker, the amplitude of the conversion coefficient peak gradually decreases. The amplitude of the elastic dissipative coefficient peak, in the other hand, gradually increases with the increase of the thickness of membrane since the conversion energy partially converts to elastic energy due to the increase of the strain energy [22].
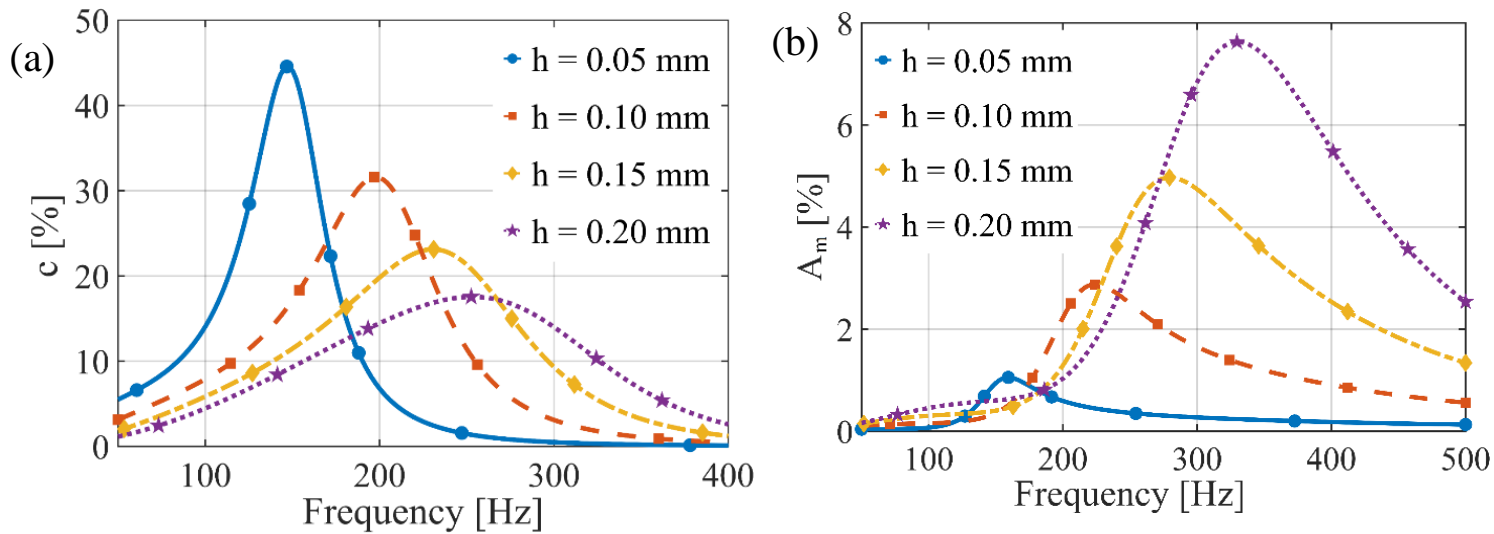

Figure 2.4. The effect of the membrane's thickness on the conversion coefficient of (a) the harvester and the elastic dissipative energy of (b) the membrane.

The contribution of the mass' dimensions on the conversion coefficient is presented in the Figure 2.5(a) and Figure 2.5(b). In the study, the external load $R_{l}$ is set to be zero and the other parameters are the same as listed in Table 2.1. It is observed that with the increase of the mass radius $b$ or thickness $t$, the resonant frequencies of the harvester will decrease due to increase of the mass weight leading to a corresponding shift in the conversion coefficient 
peak's position. While the conversion coefficient peak is clearly lowered when the mass' thickness $t$ is increased, it will initially increase when the mass' radius $b$ increases, then reach the best value at $b=2.5 \mathrm{~mm}$, and continually decrease afterwards. Note that, to the best of our knowledge, the best conversion coefficient peak of $48 \%$ observed at $b=$ $2.5 \mathrm{~mm}$ is much larger than conversion coefficients possible in a thin MAM. Furthermore, the corresponding absorption peak is nearly $50 \%$, equal to the limit of the absorption capacity for a thin MAM harvester [22].
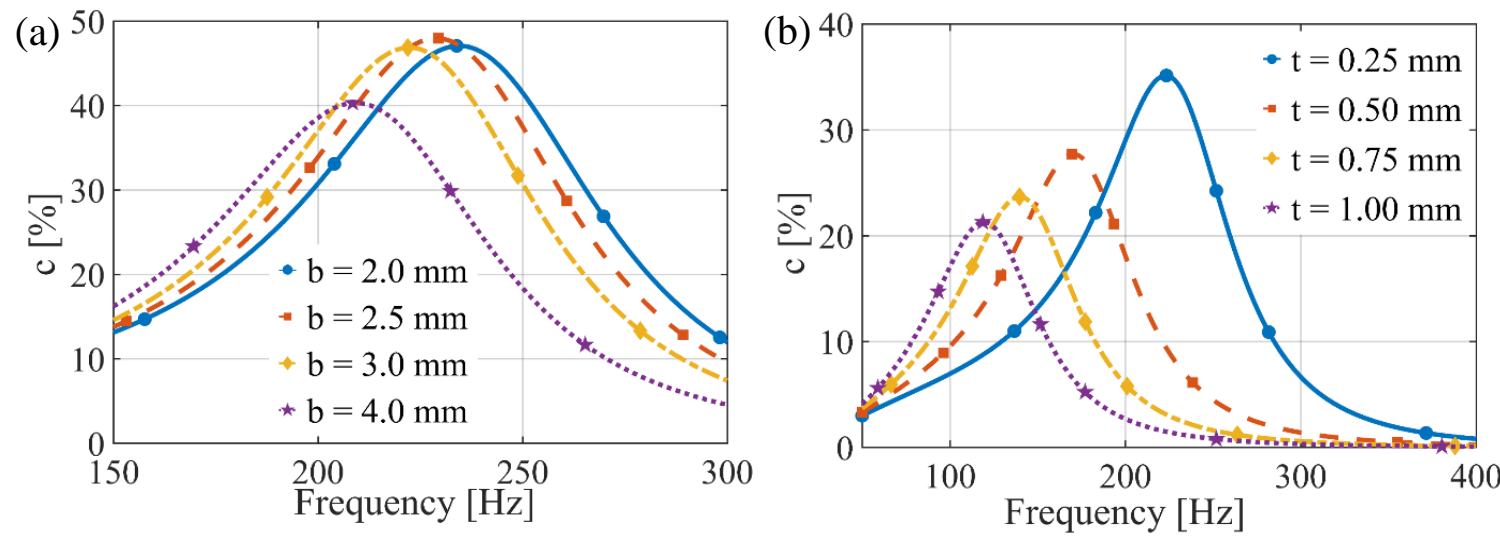

Figure 2.5. The effect of the mass' dimensions on the conversion coefficient of the harvester.

dissertation
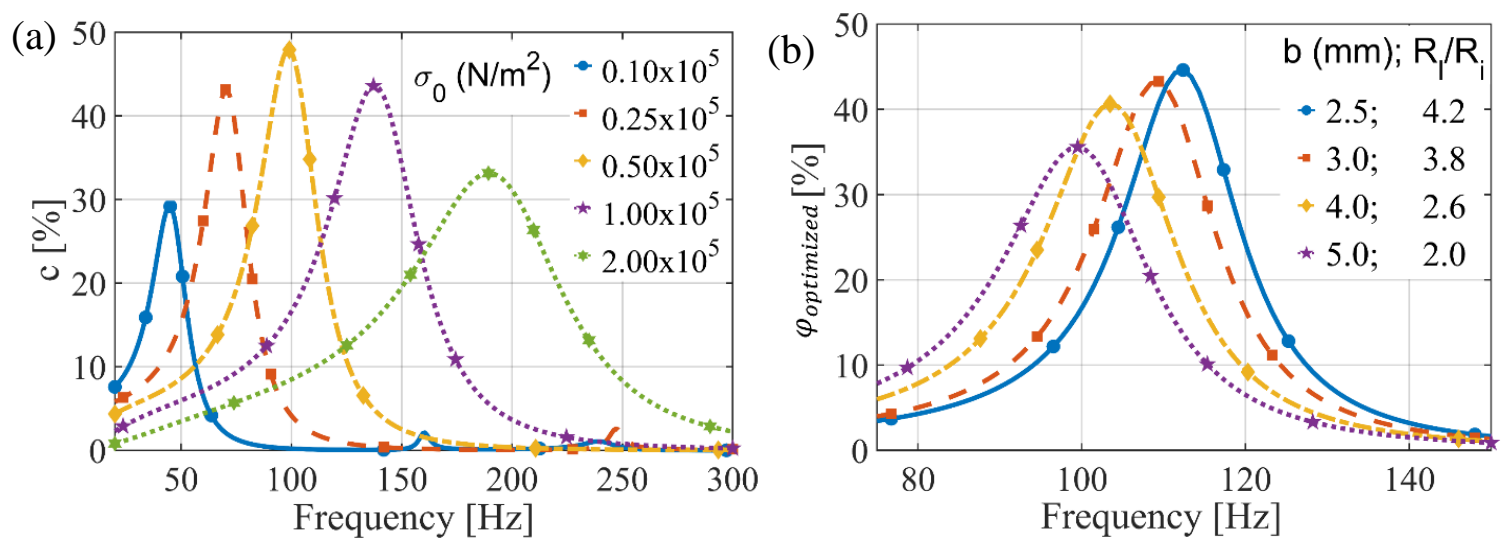

Figure 2.6. (a) - The effect of the membrane's prestress on the conversion coefficient; (b) - The optimized harvesting coefficient of the harvester when $\sigma_{0}=0.5 \times 10^{5} \mathrm{~N} / \mathrm{m}^{2}$.

Based on these parameter analyses, we optimized the energy harvester design. Using the above insights, the prestress $\sigma_{0}=0.5 \times 10^{5} \mathrm{~N} / \mathrm{m}^{2}$ is selected. While other parameters are 
kept the same as listed in the Table 2.1, two parameters (the mass' radius and the external load $R_{l}$ ) are varied to find the optimized harvesting coefficient. Figure 2.6(b) shows the optimized harvesting coefficients corresponding to several values of the given mass radius $b$ and optimized value of the external load $R_{l}$. We observed that the maximum harvesting coefficient peak is $36 \%$ while the possible capacity of the harvester is just $50 \%$.

In this dissertation, the MAM harvester is based on axisymmetric model, which can bring us elegant analytical solutions for both homogeneous and inhomogeneous problems. Based on these, the parameter effect and optimization can easily be conducted. For other type of structure such as rectangular MAMs or MAMs with multiple attached masses, although the governing equations and modeling of the magnet force are mostly the same to the case of axisymmetric model, the approach to solve them may need to employ the point-matching method [21, 22].

\subsection{Conclusion}

In this dissertation, an analytical model of a magneto-based membrane-type acoustic metamaterial harvester is proposed, which shows substantial improvement in all absorption, conversion, and harvesting coefficients. By investigating the contribution of the geometric parameters and membrane's prestress on the acoustic performance of the harvester, it is shown that the absorption can reach the absorption limit of a thin MAM (50\%), the conversion coefficient can reach to $48 \%$; and the harvested coefficient can also reach to a very impressive number, $36 \%$. Furthermore, the theoretical analysis has been well validated by comparing to numerical simulation results generated using the commercial code COMSOL Multiphysics. These results lay a strong foundation for 
developing acoustic metamaterial harvesters and show tremendous application potential for the proposed MAM harvester. 


\section{Chapter 3. Membrane-type Acoustic Metamaterial Panel}

\subsection{Introduction}

Low-frequency sound reduction always challenges scientists due to the limited acoustic performance of traditional materials, whose design and performance are limited by laws of nature, such as mass density, quarter wavelength laws, and so forth [44-49]. Most of the traditional sound proofing walls therefore are either cumbersome or sturdy to exploit the stiffness control region [50-52]. New approaches to control the low-frequency sound propagation in the form of new materials are extremely desirable.

Recently, acoustic metamaterials have been gaining attention, showing counter-intuitive properties that are not found in nature, such as negative mass density, negative bulk modulus, double-negative materials, and so forth [1, 52-56]. Realization of a lightweight acoustic panel possessing high transmission loss (TL) at low frequencies is one of the most promising potential applications of the acoustic metamaterials. Yang et al. [1] have suggested that a membrane-type acoustic metamaterial (MAM) based on a thin and soft membrane with an attached mass in the center supports negative effective mass density within the frequency region slightly before the anti-resonant frequency. At the antiresonance where the MAM's average normal displacement vanishes, the transmitted sound is dramatically suppressed, consequently making the MAM an acoustically hard wall [57-59]. The working region therefore can be tuned by adjusting the resonant frequencies of the MAM [57, 60, 61]. However, most of the existing results are so far limited in the unit cell scale and quite difficult to be transferred to large-scale products since the fixed rim or periodic boundary conditions employed in unit cell studies can hardly be practiced 
in large-scale products. The support frame for a large-scale panel inevitably suppresses the low-frequency sound reduction performance due to the emergence of additional global resonances from the frame. Aside from the MAM, plate-type acoustic metamaterials have also been proposed for noise reduction applications. Although a plate-type acoustic metamaterial unit cell exhibits great sound reduction performance [60], its performance drops significantly in the large-scale panel configuration [62]. The synergetic coupling in a plate-type acoustic metamaterial supercell, supporting four weakly coupled resonances (TL peaks), was proposed for low-frequency sound reduction [10]. However, its improvement over the mass density law is still limited and only demonstrated within narrow bands. Other plate-type acoustic metamaterial designs by embedding coupled cavities within a unit cell [63] realized TL enhancement based on coupling modes. The performance of this design is mainly affected by the stiffness control region; the observed benefit given by the coupling only takes place within a narrow band. Therefore, in order to achieve optimal sound reduction performance in a large-scale metamaterial panel, optimized unit cell performance and suppression of the panel vibration are essential. In this dissertation, we present an acoustic panel made of a multicelled double-layer membranetype acoustic metamaterial (DMAM) array to achieve ultra large sound transmission loss. The proposed panel is fully characterized by the theoretical models of the MAM and DMAM, which are numerically validated. The supporting frame of the proposed panel is designed to be lightweight and possess high bending stiffness in order to increase the frequency of its vibration and thus suppress the adverse effect on the overall sound reduction performance within low-frequency regions. The frame vibration is also investigated to optimize the working region of the panel. By choosing proper design 
parameters, excellent transmission loss performance can be obtained in the panel configuration at low frequencies, which are also evidenced by experimental testings.

\subsection{Double layer acoustic metamaterial unit-cell design}

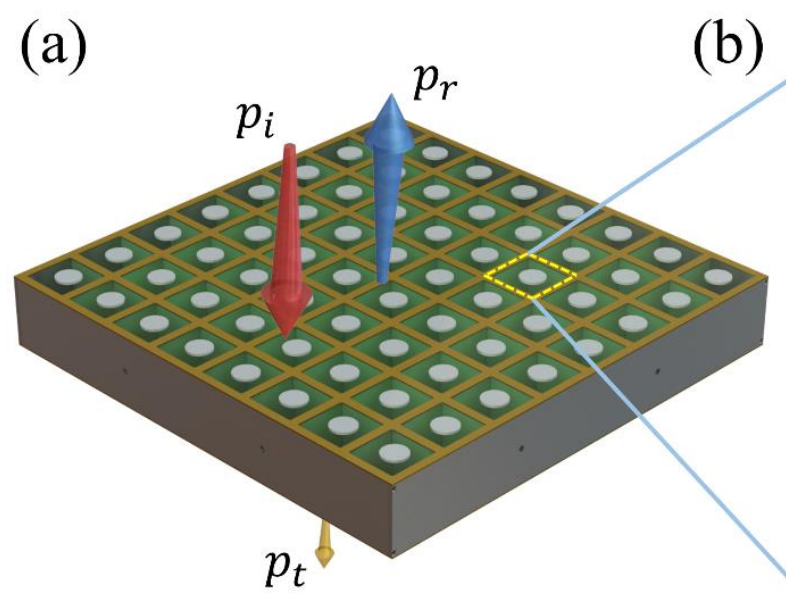

(d)
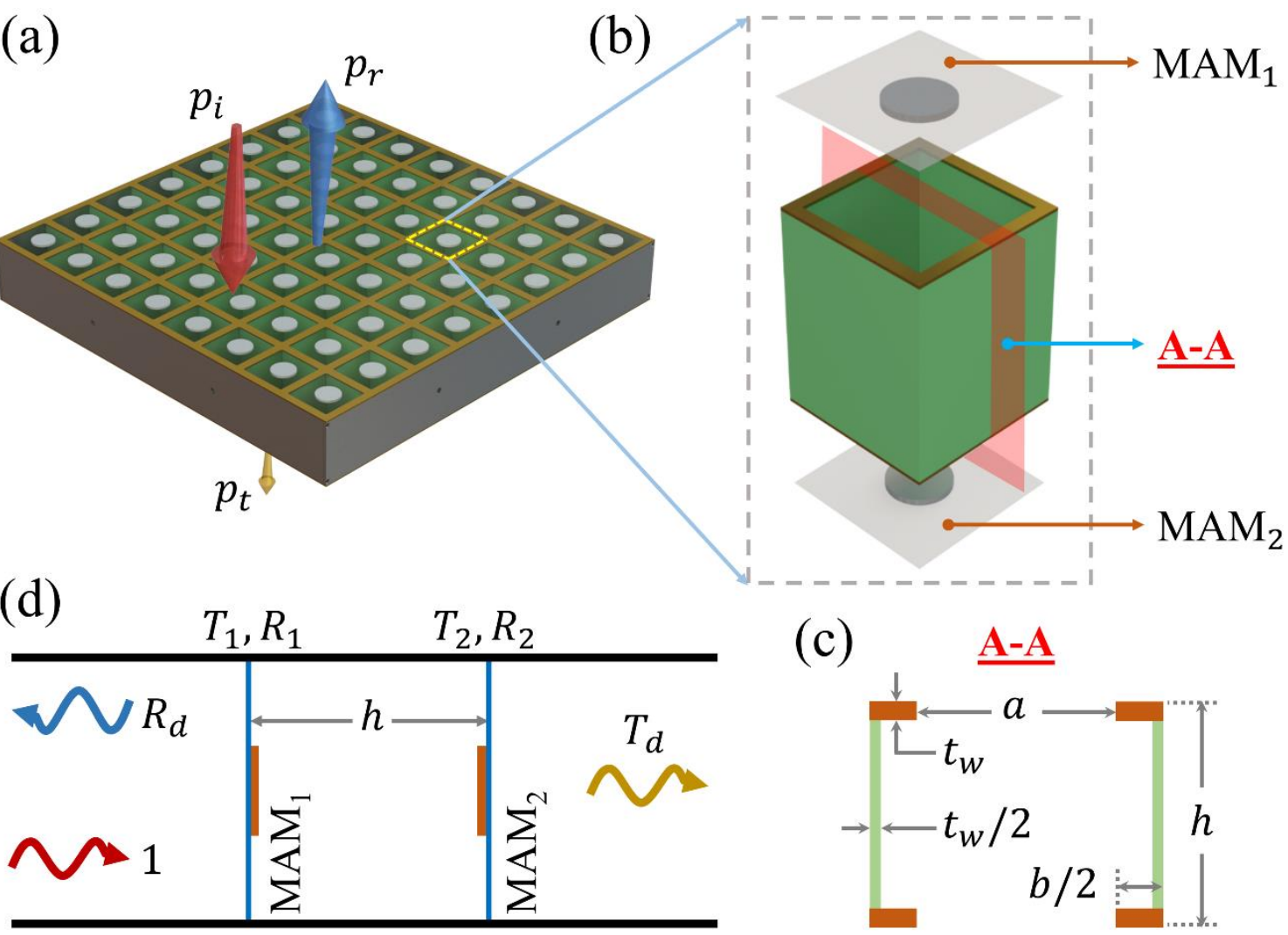

Figure 3.1. (a) Schematic of the proposed large-scale acoustic panel design. The incident sound $\left(p_{i}\right)$ wave is mostly reflected $\left(p_{r}\right)$, resulting in strongly suppressed sound transmission $\left(p_{t}\right)$. (b) Detailed view of the double-layer membrane-type acoustic metamaterial unit cell. It is assembled from two membrane-type acoustic metamaterial unit cells and the gridded-like elastic frames. (c) Sectional view of the frame. All the geometric parameters are indicated. The parts of different colors are all made of aluminum. (d) Schematic

of the acoustic model of a double-layer membrane-type acoustic metamaterial unit cell in the tube environment. An incident sound wave of normalized amplitude is considered. $T_{d}$ and $R_{d}$ denote the transmitted and reflected waves, respectively.

The design of our proposed acoustic panel is illustrated in Figure 3.1(a). It is composed of 8x8 DMAM unit cells supported by an elastic frame. All geometrical and material properties of the panel are listed in Table 3.1. Each unit cell comprises two MAM components, connected through an elastic frame, as indicated in Figure 3.1(b). As will be discussed later, we employ the I-beam design for the support frame since it generates large 
bending stiffness and therefore exhibits resonance effect at higher frequencies [see Figure 3.1(c)]. Note that the global resonances mainly depend on the height of the I beam (h). The thickness $t_{w}$ barely affects the resonances due to the fact that the bending stiffness is a cubic function of $t_{w}$.

We start with the theoretical investigation on the DMAM unit cell. Since the supporting frame panel has non-trivial thickness $(\mathrm{h}=63 \mathrm{~mm})$, resulting in large bending stiffness [64], the DMAM unit cells can be considered to have nearly fixed boundary conditions on the lateral sides in the unit cell analysis. When a noise source is located far from the panel, the incoming noise sound can be treated as normal incidence. As theoretically sketched in Figure 3.1(d), under the normalized incidence $\left(p_{i}=e^{-i k x}\right)$, the transmission and reflection coefficients are defined as $[65,66]$

$$
T_{d}=\frac{\vartheta T_{1} T_{2}}{1-R_{1} R_{2} \vartheta^{2}}
$$

and

$$
R_{d}=R_{1}+\frac{T_{1}^{2} R_{2} \vartheta^{2}}{1-R_{1} R_{2} \vartheta^{2}}
$$

respectively, where $\vartheta=e^{-i k x},\left(T_{1}, R_{1}\right)$, and $\left(T_{2}, R_{2}\right)$ are the phase shift factor between two MAMs, transmission and reflection coefficients of the $\mathrm{MAM}_{1}$ and $\mathrm{MAM}_{2}$, respectively. These coefficients can be obtained from theoretical [57, 58], simulation [67], or experimental [4] results for a single MAM unit cell. In this dissertation, to keep the design and fabrication simple, two identical MAM components are chosen, i.e., $\mathrm{T}=\mathrm{T}_{1}=\mathrm{T}_{2}$ and 
$\mathrm{R}=\mathrm{R}_{1}=\mathrm{R}_{2}=1-\mathrm{T}[58]$. Then, the TL of the MAM $\left(T L_{s}\right)$ and DMAM $\left(T L_{d}\right)$ unit cells can be defined as

$$
\begin{aligned}
& T L_{s}=-20 \times \log _{10}|T| \text { and } \\
& T L_{d}=2 \times T L_{s}+20 \times \log _{10}\left|1-(\vartheta R)^{2}\right|,
\end{aligned}
$$

respectively, which reveals that in the vicinity of the antiresonances $(\mathrm{R} \rightarrow 1), T L_{d}$ is at least twice as high as $T L_{s}$ [65]. Therefore, in order to achieve optimized sound reduction performance in the DMAM unit cell, the TL of the MAM component must be optimized. In addition, $T L_{d}$ reaches dips and peaks at the MAM resonances $(|\mathrm{T}| \rightarrow 1)$ and antiresonances $(|\mathrm{T}| \rightarrow 0)[1,68]$, respectively. In other words, the location of MAM TL dips and peaks will be preserved in the DMAM unit cell. The antiresonance at low frequencies is a result of the hybridization between the first resonance mode (named mass mode where the mass oscillates strongly) and the second resonance mode (named membrane mode where the mass is stationary, but the membrane vibrates) [see points A, F and C in Figure 3.2(b) for mass mode, membrane mode and hybrid mode, respectively]. Therefore, it is also called hybrid mode [4] and locates closer to the first resonance mode than the second one does since the former is stronger in intensity. The working region defined as the region centered at the anti-resonance and covering high TL spectrum can be tuned by adjusting the first two resonances of the MAM component. In other words, the working region mainly depends on the spectral distance between two modes. 


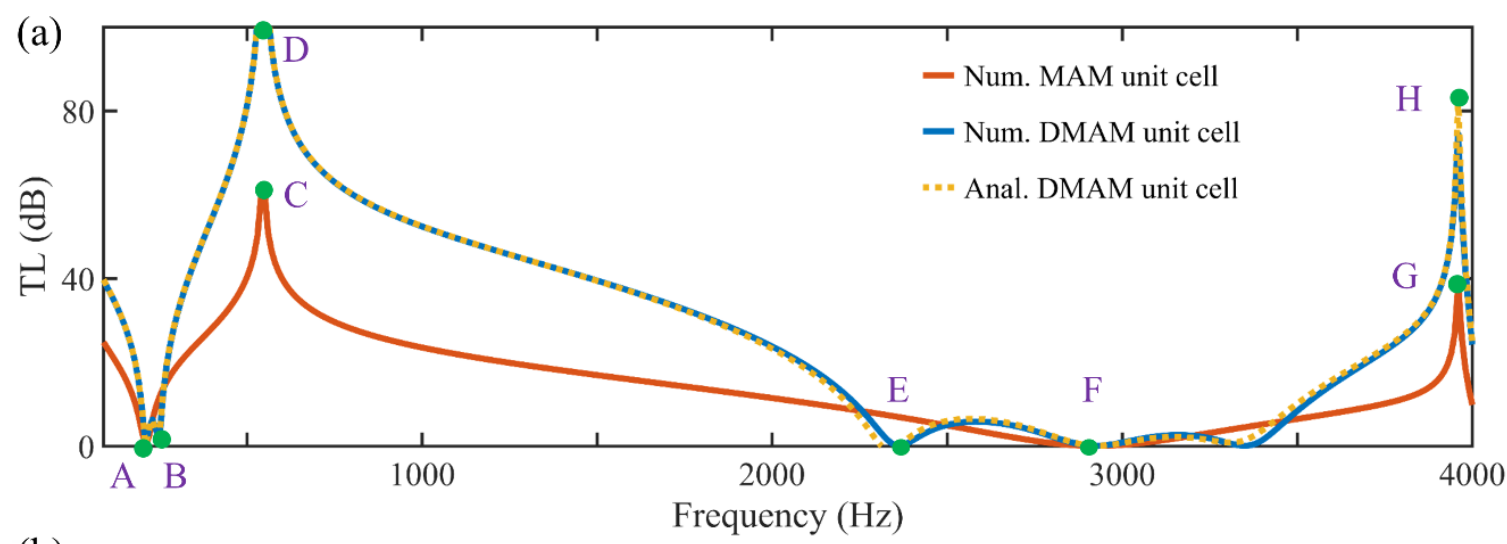

(b)

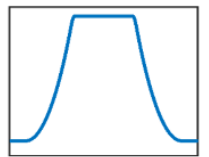

$\mathrm{A}_{\text {MAM }}$ $(211 \mathrm{~Hz})$

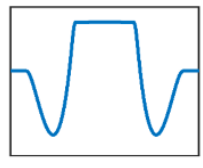

$\mathrm{C}(545 \mathrm{~Hz})$

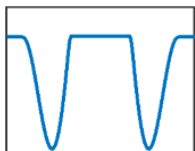

$\mathrm{F}_{\mathrm{MAM}}$ $(2925 \mathrm{~Hz})$

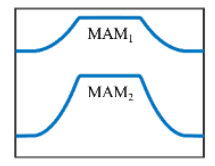

A DMAM $(211 \mathrm{~Hz})$

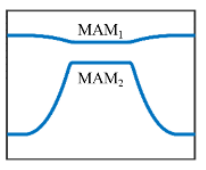

$\mathrm{B}(250 \mathrm{~Hz})$

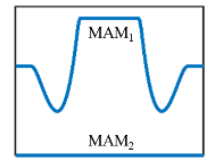

$\mathrm{D}(555 \mathrm{~Hz})$

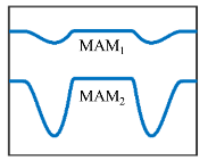

$\mathrm{E}(2360 \mathrm{~Hz})$

Figure 3.2. (a) Transmission loss spectrum of the MAM/DMAM unit cells from both simulation (Num.) and theoretical (Anal.) analysis. (b) Mode shapes of the MAM/DMAM unit cell at the peaks and dips.

Table 3.1. The acoustic panel's parameters

\begin{tabular}{llc}
\hline Element & Properties & Value \\
\hline Unit-cell size & Width x Height & $50 \mathrm{~mm} \times 50 \mathrm{~mm}$ \\
& Thickness & $63 \mathrm{~mm}$ \\
Elastic membrane & Young's modulus & $3 \mathrm{GPa}$ \\
& Mass density & $960 \mathrm{~kg} / \mathrm{m} 3$ \\
& Poisson's ratio & 0.48 \\
& Thickness & $0.076 \mathrm{~mm}$ \\
& Loss factor & $796 \mathrm{~Pa} \cdot \mathrm{s}$ \\
Pass & Prestress & $5 \mathrm{MPa}$ \\
& Material & Aluminum \\
& Diameter & $21.59 \mathrm{~mm}$ \\
Frame & Thickness & $2 \mathrm{~mm}$ \\
& Material & Aluminum \\
& Web and flange thickness: $t_{w}$ & $1 \mathrm{~mm}$ \\
& Grid-cut size: $a \times a$ & $42 \mathrm{~mm} \times 12 \mathrm{~mm}$ \\
\hline
\end{tabular}


In the MAM, since the membrane is very lightweight and hardened by pre-tension $[1,67]$, the second resonance mode's frequency can be tuned far away from the first resonance mode [57, 69], mainly affected by the attached mass. In this way, a broad working region is expected for the MAMs with large pre-tension. Figure 3.3(a) shows the TL of the MAM component under different membrane pre-tension conditions. It can be confirmed that when the pre-tension is increased, the first resonance mode is slightly shifted to higher frequencies, while the second resonance mode shifts in a more significant way. In other words, increasing the pre-tension leads to a greater spectral distance between two modes and hence a larger working region. Therefore, adjusting pre-tension is an efficient way to control the working region of the MAM components while the weight and shape remaining unchanged. Considerably large pre-tension may challenge the fabrication and longevity due to the creeping of the MAM over the time. In this dissertation, 5 MPa pre-tension on the membrane is selected in the DMAM unit cell. With such a MAM configuration [red curve in Figure 3.3(a)], one dip and one peak appear corresponding to the first MAM resonance and antiresonance located at 211 and $545 \mathrm{~Hz}$, respectively. 

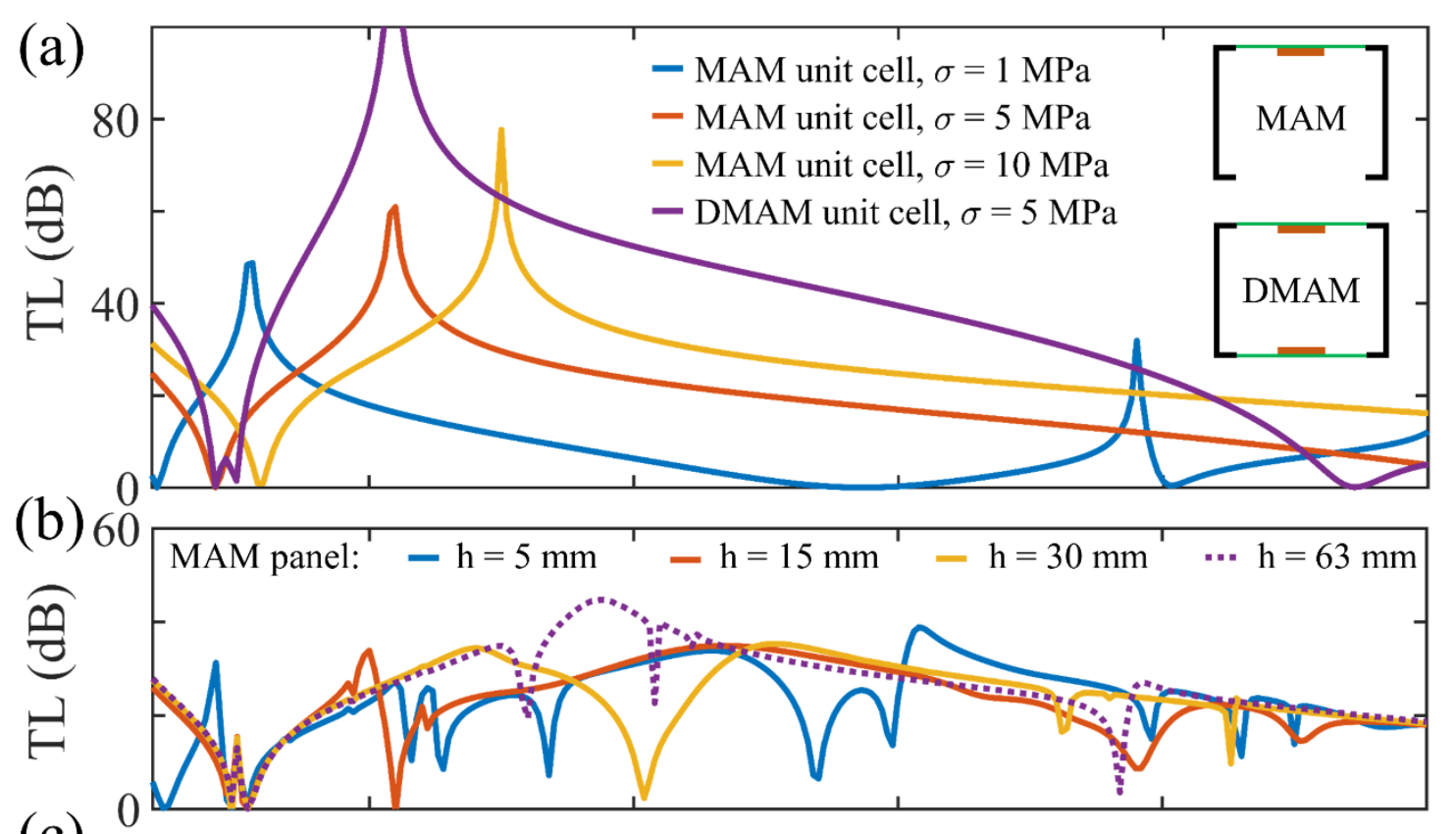

(c)

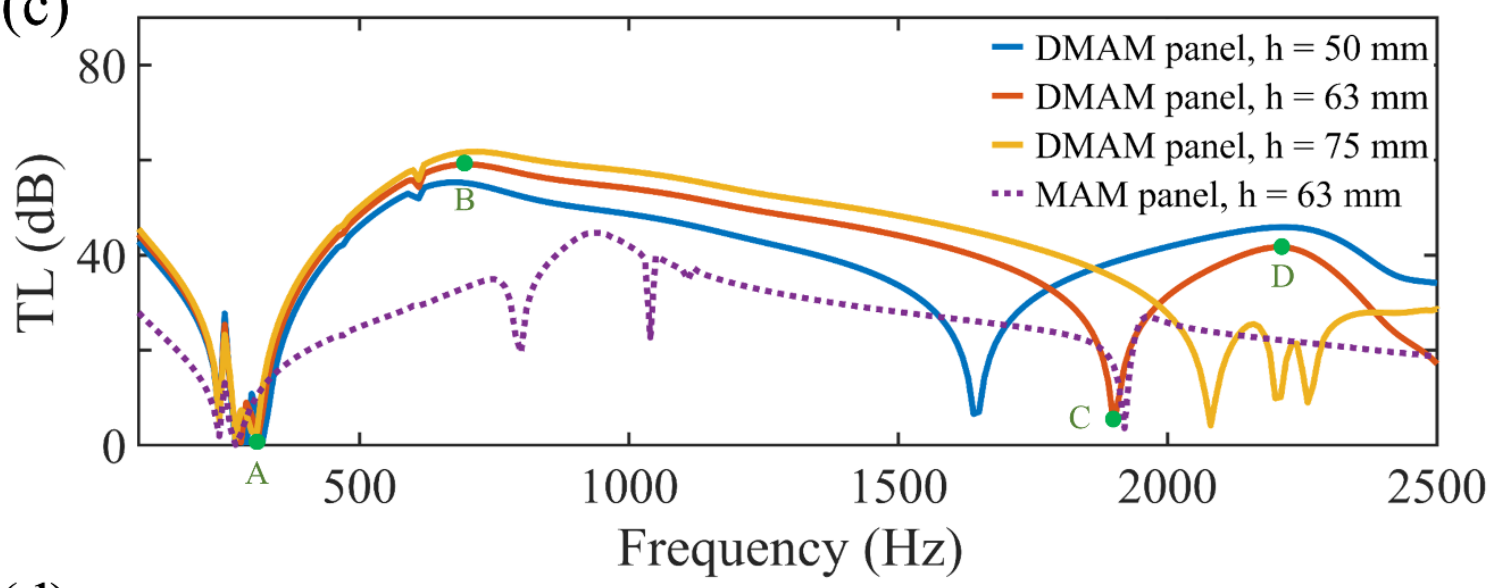

(d)

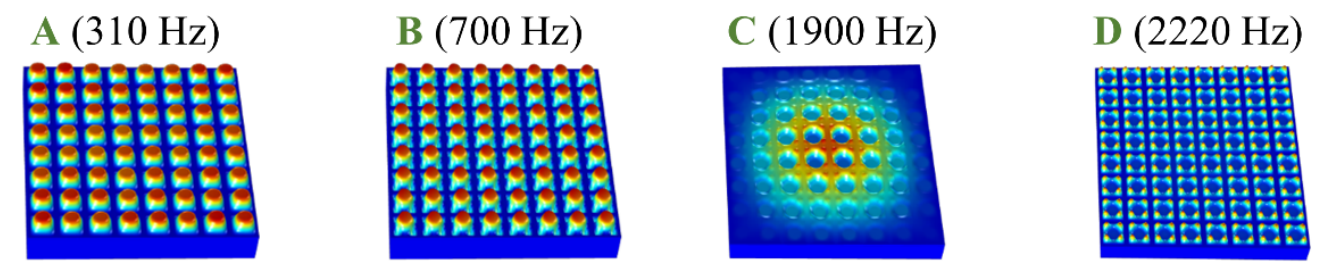

Figure 3.3. (a) - Transmission loss spectra of the single MAM unit cell under different membrane pretension conditions ( $\mathrm{r}$ ) and the DMAM unit cell comprised from two identical MAMs under a pre-tension of $5 \mathrm{MPa}$. Comparison of TL spectra of (b) MAM panels and (c) DMAM panels with different thicknesses of the panel thickness when a pre-tension of $5 \mathrm{MPa}$ is employed on the membranes. (d) The mode shapes of the DMAM panel of the highlighted peaks and dips.

The DMAM unit cell constructed by two selected MAMs separated by $63 \mathrm{~mm}$ is studied and the corresponding result is presented by the purple curve in Figure 3.3(a) purposely to compare its performance with the single MAM component. Obviously, the TL of DMAM 
unit cell $\mathrm{TL}_{\mathrm{d}}$ is significantly higher than that of the single MAM component (red curve) $\mathrm{TL}_{\mathrm{s}}$. Within most regions except for the vicinity of the MAM resonance, $\mathrm{TL}_{\mathrm{d}}$ is nearly twice as high as $\mathrm{TL}_{\mathrm{s}}$ and the peak and dip of the MAM are preserved in the DMAM configuration. These observations confirm the advantage of DMAM unit cell over the single MAM component discussed previously. Other than the TL dips inherited from the MAM component, additional dips at $250 \mathrm{~Hz}, 2360 \mathrm{~Hz}$, and $3350 \mathrm{~Hz}$ as observed on Figure 3.2 emerge in the DMAM configuration. In fact, they are a consequence of Fabry-Perot resonances between the two MAM components. In summary, there still exists a continuous broadband TL spectrum of at least $25 \mathrm{~dB}$ covering the range from 0.28 to $2 \mathrm{kHz}$.

\subsection{Acoustic metamaterial panel}

Generally, acoustic panels, constructed by attaching unit cells on a grid-like elastic frame, have low bending stiffness if the unit cell is thin, for instance, a MAM unit cell held on a thin frame $[10,60,61]$. In real applications, the panel edges are often constrained, leading to several panel vibration modes distributed at low frequencies. These vibration modes cause strong but unavoidable interference of these modes on the local modes of unit cells. Consequently, the design performance of the unit cell is heavily undermined [60, 63]. Therefore, in addition to the need of excellent performance of unit cell, a high bending stiffness of the panel frame is another requirement for optimized sound reduction performance. Figure 3.3(b) illustrates the TL spectra of the MAM panels ( $\sigma=5 \mathrm{MPa})$ with different panel thicknesses. Clearly, when the thickness is small, multiple TL dips, which do not exist in the unit cell analysis, appear within the low-frequency region. With the increased thickness, the dips exhibit blueshifts as the panel vibration resonances go to higher frequencies due to the higher bending stiffness the panel possesses. Especially, 
when the thickness is $63 \mathrm{~mm}$, the performance of the unit cell within the frequency region of interest is nearly conserved in the panel configuration with the dampening and blueshift of the TL peak.

(a)

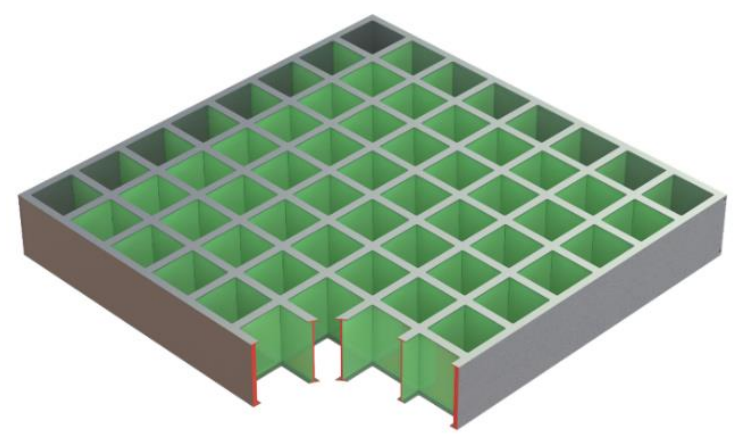

(b)

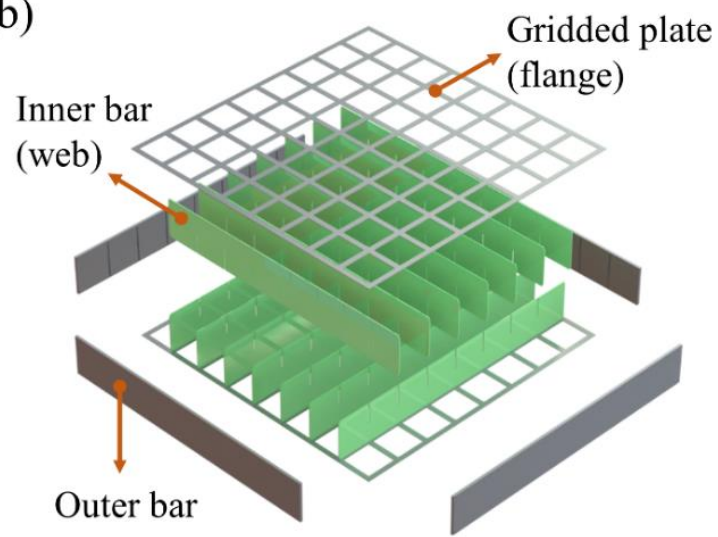

Figure 3.4. (a) Schematic illustration of the cut away DMAM panel frame. (b) Exploded view of the DMAM panel frame.

Similarly, a DMAM panel with an appropriate thickness is expected to perform better than the corresponding MAM panel by well preserving the performance of the DMAM unit cell. To examine and optimize the performance of the DMAM panel, we conduct parametric study on the thickness of the panel frame. As shown in Figure 3.3(c), the TL of the DMAM panel is much higher than that of the single MAM. The TL of the DMAM unit cell [purple curve in Figure 3.3(a)] remains in the panel configuration, especially on the left side of the TL peak (point B). In contrast, the TL after point B is lowered when decreasing the panel frame thickness. For the chosen thickness of $63 \mathrm{~mm}$, the TL dip (point C) is induced by the first-order panel vibration resonance, as can be realized through the mode shape presented in Figure 3.3(d) where the frame oscillates strongly. Interestingly, an extra TL peak (point D) emerges thanks to the panel vibration resonance, bringing satisfactory sound reduction performance within the region where the TL is trivial in the unit cell analysis [purple curve in Figure 3.3(a)]. Points A and D show the first and second resonance modes of the MAM, 
respectively [see Figure 3.3(d)]. While the former manifests as a dip as in the DMAM configuration, the latter weakly couples with the vibration of the frame to form a peak. Figure 3.3(d) also shows that the mode shape at point B resembles the anti-resonance of the MAM of which the locally optimal performance is undermined and gets shifted to the higher frequency $(545-700 \mathrm{~Hz})$ due to the interference with the vibration of the panel. Due to the large bending stiffness, the panel frame vibrates in a much smaller amplitude than the mass and membrane do at points B and D. Therefore, the local mode shapes are dominant in the coupling mode shapes to produce TL peaks, as observed in Figure 3.3(d). It is noteworthy that due to the effect of the global mode, the first resonance mode shifts from 211 to $310 \mathrm{~Hz}$. In short, despite the presence of the mode shifting and the global mode, a broadband TL region at $2559 \mathrm{~dB}$ is still noticeable from 0.35 to 2.85 and 1.85 to $2.4 \mathrm{kHz}$ in the panel. 

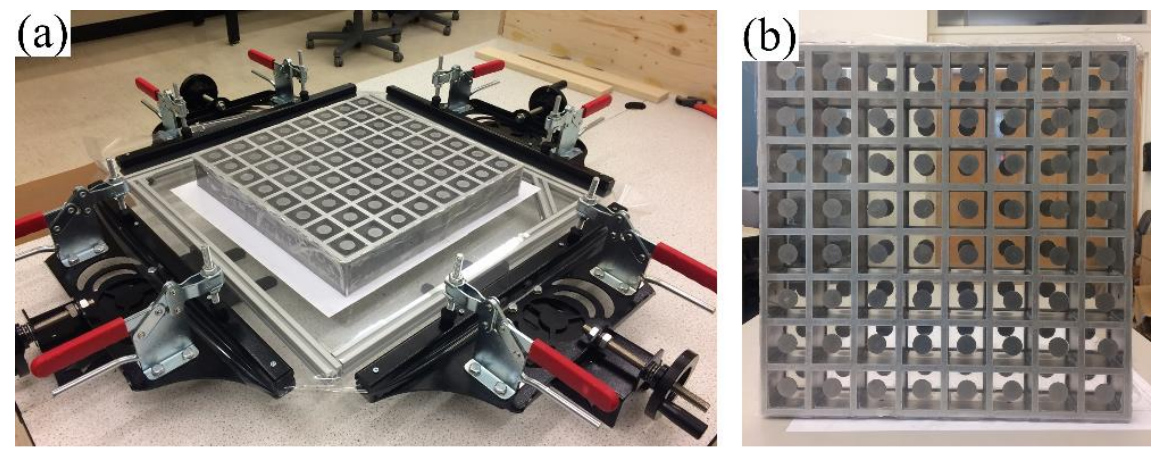

(c)

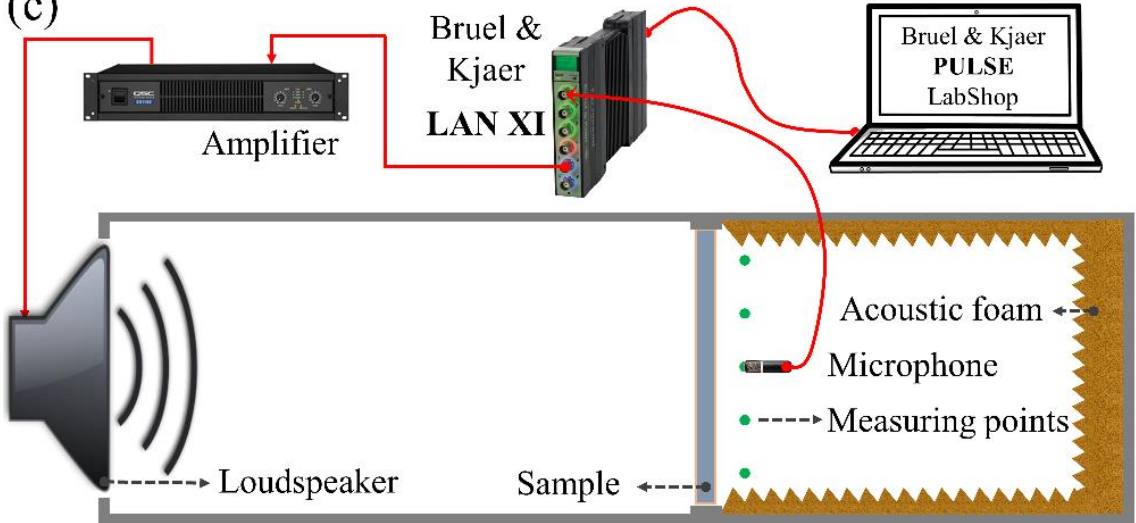

(d)

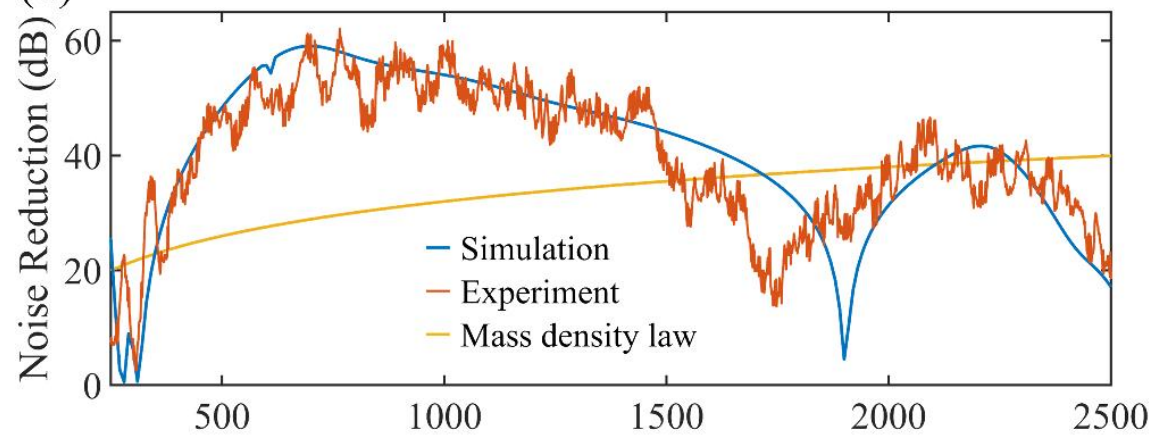

Frequency $(\mathrm{Hz})$

Figure 3.5. (a) Photograph of stretching the membrane in the acoustic panel. The pretension on the membrane can be easily adjusted. (b) Photograph of the large-scale double-layer membrane-type acoustic metamaterial-based panel. (c) Schematic illustration of the experimental setup. The foam layer within the tube cross section is of a thickness of 8 inches. A 2 inch-thick air cavity is arranged next to the foam. (d)

Comparison between the simulation and experimental results for the sample shown in (b). The noise reduction performance according to the conventional mass density law is also presented for illustrating how well the large-scale double-layer membrane-type acoustic metamaterial-based panel performs.

To illustrate the practical application, we fabricate an acoustic panel sample following the parameters provided in Table 3.1 [Figure 3.5(a)]. The panel frame is an assembly of web bars and flange plates [see Figure 3.4], which are designed deliberately and fabricated by a CNC machine. They are then bonded together by super glue. The membranes are cut 
from a polyethylene clear sheet, and the pre-tension is applied using a stretching machine; see Figure 3.5(a). This ensures that the pre-tension strengths in all unit cells are uniform and thus suppress couplings between neighboring units. The circular masses are speedily produced on a punching machine and attached to the membranes with super glue. The finalized DMAM panel is shown in Figure 3.5(b). To experimentally test the sound insulation performance of the sample, small semi-anechoic chamber as described in Figure 3.5(c) is fabricated. The plane wave-like noise sound is generated from a $16 \mathrm{in.} \mathrm{loudspeaker}$ placed far away from the sample at the inlet of the chamber. An acoustic foam of thickness 8 inches, backed by a 2 inch-thick air cavity, is then installed on the end of the receiving chamber. Some thinner foam layers are then attached on the inner side walls to make the outlet an anechoic chamber. All the employed foams can efficiently absorb the sound waves even within the low-frequency range [see Figure 3.6 for the setup and measurement result of the absorption of acoustic foam]. A commercial software Bruel Kjaer PULSE Labshop is then utilized to conduct the measurement. We use Labshop, through the LAN $\mathrm{XI}$, to generate a noise signal ranging from 0.08 to $3.2 \mathrm{kHz}$. The signal is later amplified by a power amplifier before being sent to a high-power loudspeaker. A $1 / 2$ inch Bruel Kjaer free-field condenser microphone (type 4966-H-041) with a high-temperature pre-amplifier (type 1706) is used to measure the pressure amplitudes inside the anechoic chamber on a matrix of $5 \times 5$ points on a plane 4 in. distance from the sample. The measurement is proceeded in two steps [70]. First, the background pressure amplitude is measured in the absence of the sample. Later, the transmitted pressure amplitude is measured after the sample is installed. The noise reduction is then calculated from the pressure measured. Besides the experimental result, the simulation result and mass density law are also 
presented for comparison, as shown in Figure 3.5(d). The experimental result shows excellent agreement with the simulation result and deeply breaks the mass density law in the region $0.37-1.5 \mathrm{kHz}$. TL exceeding $20 \mathrm{~dB}$ at almost all frequencies within the range $0.32-2.5 \mathrm{kHz}$ and that ranging $40-59 \mathrm{~dB}$ from 0.45 to $1.48 \mathrm{kHz}$ were recorded. More importantly, the proposed DMAM panel with frame vibration suppressed outperforms the acoustic panel previously reported in which the efficient sound insulation range only covers $50-1000 \mathrm{~Hz}$ [2]. Moreover, the acoustic panel is optically transparent as well by using transparent plastic sheets as the membranes.
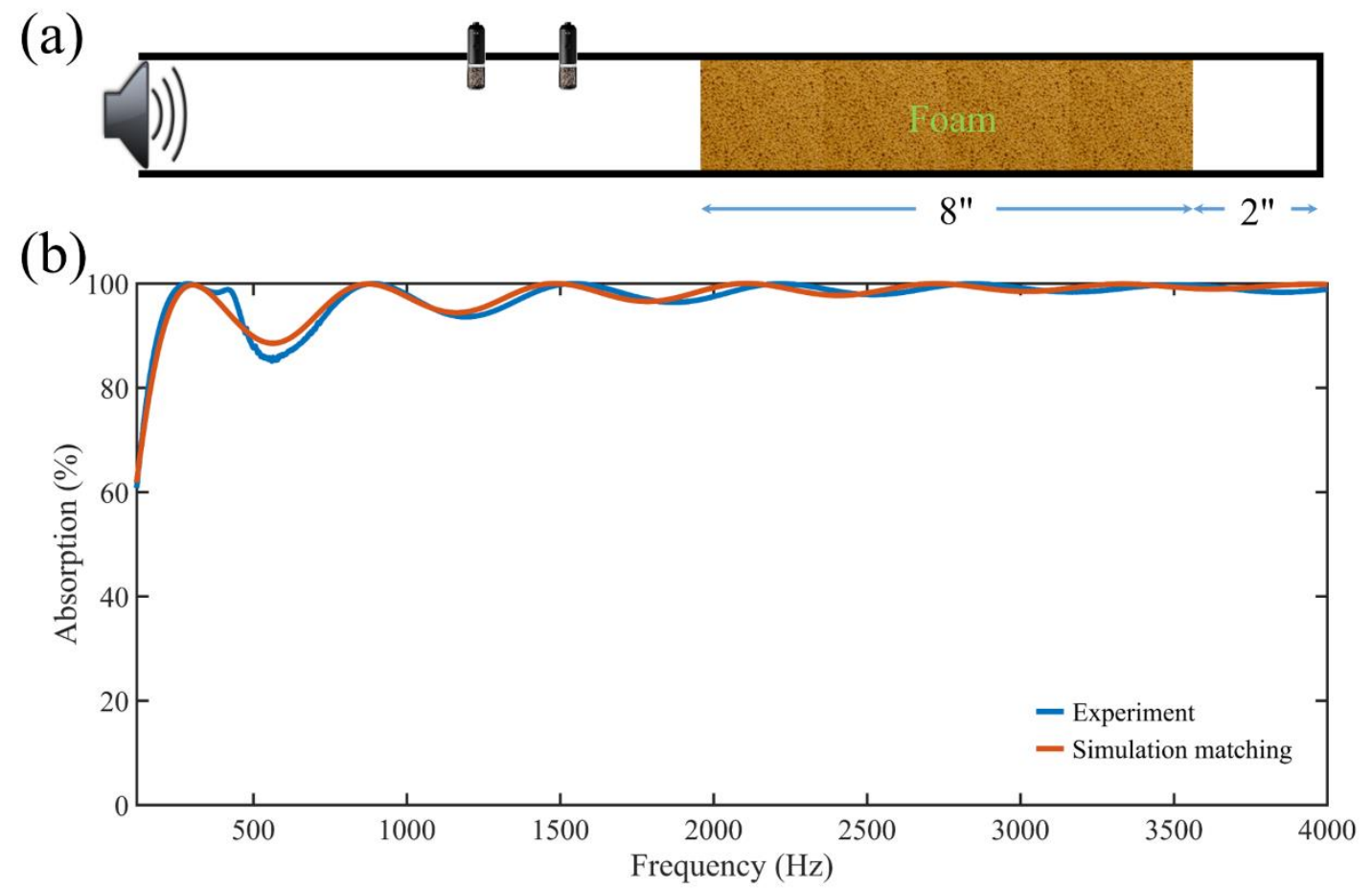

Figure 3.6. (a) Setup for measuring absorption coefficient in a commercial impedance tube. (b) The experimental and simulation results.

\subsection{Conclusion}

In summary, the double-layer membrane-type acoustic metamaterial has been proposed and studied thoroughly by both theoretical and numerical analyses, which indicates that the 
transmission loss of the proposed DMAM unit cell is at least twice as high as that of the MAM configuration around the antiresonance. An acoustic panel based on an 8x8 DMAM array is then proposed to achieve broadband noise reduction. The experimental result shows excellent transmission loss spectrum overall exceeding $20 \mathrm{~dB}$ within the range $0.32-$ $2.5 \mathrm{kHz}$. Specifically, transmission loss ranging 40-59 dB within $0.45-1.48 \mathrm{kHz}$ deeply breaks the mass density law. In addition, the proposed panel is almost optically transparent, which is essential from an esthetic standpoint. We believe that the proposed design and the associated theoretical model may pave the way for designing highly efficient and lightweight acoustic panels and barriers with broadband operations at low/middle frequencies. 


\section{Chapter 4. Sonic Crystal Based Acoustic Barrier}

\subsection{Introduction}

Ultra-broadband sound reduction schemes, which cover noise spectra in living and working environments, are in high demand in both academic and industrial communities [70-74]. Traditional acoustic barriers, which are categorized as either acoustic composite panels $[47,67]$ or sonic crystals $[75,76]$, have been widely employed. Their performance is fundamentally restricted especially in the low-frequency range, and heavy and bulky designs are usually required. While the former is opaque, the latter interestingly is partially optically transparent and provides ventilation. Principally, there always exists a trade-off between the open ratio, defined as the ratio of the gap between two columns to the lattice constant, and the thickness and performance of a crystal-based sonic barrier [65, 70, 77]. Previous studies have shown that in order to achieve satisfactory performance, say insertion loss commonly over at least $10 \mathrm{~dB}[70,78]$ within the range $0.3-4.0 \mathrm{kHz}$, an acoustic barrier thickness of at least $0.6 \mathrm{~m}$ is required with an open ratio of less than $27 \%$ [70, 71, 77]. These designs are essentially too cumbersome to be employed in real applications, especially in an office or working environment. The main reason for this lies in the fact that they all rely on traditional materials, such as solid, perforated or porous rods deployed on periodic structures. Therefore, new designs with novel materials or structures are necessary in order to overcome the limitations of traditional designs.

In the past two decades, acoustic metamaterials, which refer to artificial resonance-based materials, have been thought of as the most promising candidate to break the traditional materials' limitations in airborne acoustics. In particular, space-coiling metamaterial 
(SCM) emerges as a special class of metamaterials [8]. It is constructed by coiling up the space within its unit cell to form curled acoustic channels with a subwavelength crosssection. The propagating phase and amplitude can be tuned arbitrarily by adjusting the geometry of the curled channel to give rise to extreme acoustic behaviors, such as an extreme refractive index [79], double-negative media [8] and phase/amplitude modulation [80]. In addition, the SCM was reported to exhibit Fano-like profiles [8]. In general, Fano resonance, first observed by Fano [81], is a special class of coupling resonance, which, in most cases, refers to the interference of a local resonance and a background continuum. It comes with a narrow-linewidth asymmetric absorption profile, induced by the intensity interaction and phase-shift involved in inelastic scatterings of electrons in a quantum mechanical setting. In solid mechanics, Fano resonance has been discussed both theoretically and experimentally in several studies [82-84]. In acoustics, however, Fano resonances have been demonstrated visually through asymmetric transmission loss (TL) profiles in simulation and experiments $[8,11,85]$. A complete theoretical analysis of the formation of Fano resonances specifically by the interference of monopolar/dipolar modes and the background continuum is yet to be established. Two key factors involved in the formation of acoustic Fano resonance are local resonance and coupling of resonances. The former, on the one hand, is generally established by satisfying input impedance matching conditions (zero reactance) $[47,65,86]$ and exhibits extreme and unnatural effective constitutive properties $[1,55,87,88]$. The latter, on the other hand, is a consequence of interaction of multiple resonances under certain matching conditions. For instance, coupling between two modes of an acoustic resonator results in an antiresonance with high TL [1, 59] or a hybrid resonance with perfect absorption [4]. Coupling between resonances 
from multiple separate resonators also admits perfect absorption $[68,89]$ and allows the existence of multiple double-negative bands [90]. Using Fano resonance, high TL performance was only achieved within a narrow band [11, 91, 92]. Further exploiting multiple Fano resonances induced by four different Mie resonators [8], a broader band up to $1.25 \mathrm{kHz}$ was observed in numerical simulations. However, the corresponding design with a low open ratio $(20 \%)$ is still quite thick $(0.46 \mathrm{~m})$. To obtain efficient and ultrabroadband TL performance in a compact design, not only fundamental but also higherorder Fano resonances must come into play. That is to say, all of them should be involved and distributed more optimally to cover the broader frequency range of interest [93].

In this dissertation, based on multiple Fano resonances, we propose an acoustic barrier consisting of multiple SCMs to realize ultra-broadband sound reduction. We first aim to deliver a comprehensive theoretical model to characterize the SCM and the Fano resonance associated with it. The analysis involves replacing the actual SCM with an effective model, which contains an effective, straightened channel filled with slow-wave materials. The effective channel, which supports both monopolar and dipolar modes, interacts with the background continuum, giving rise to Fano resonances. Because of their coupling nature, the effective model is hence called a coupling unit throughout the research. The Fano resonances are thoroughly studied using a transfer matrix method and effective medium theory, and are fully characterized in terms of TL. Corresponding numerical simulations are then conducted to validate the theoretical prediction and also to better illustrate the Fano-resonance-based broadband sound reduction. Lastly, we construct an acoustic barrier using the proposed SCMs with different geometrical properties, and conduct experiments to demonstrate potential applications of ultra-broadband sound reduction. 


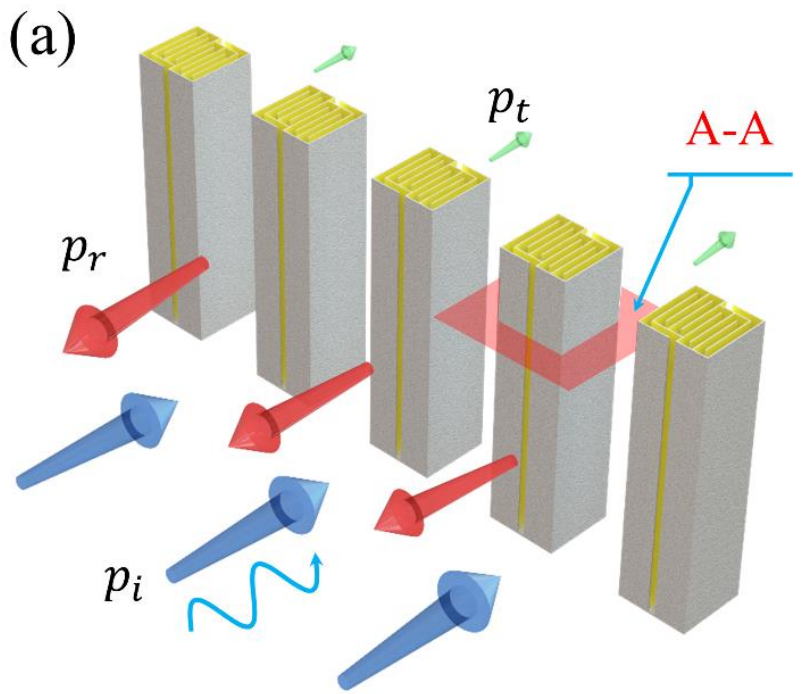

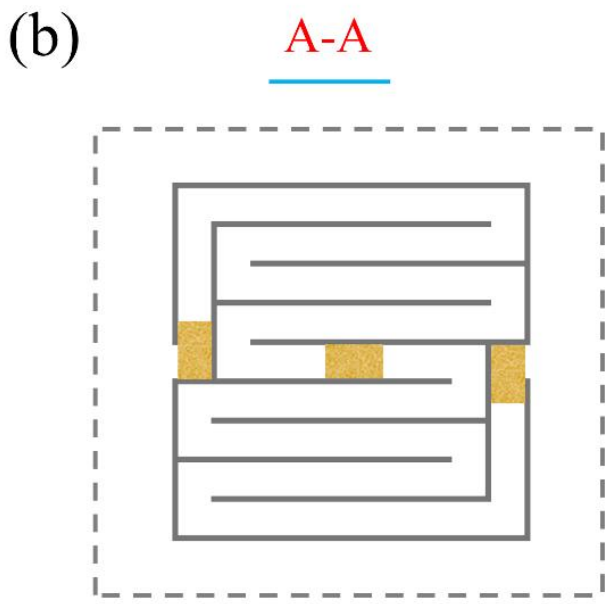

Unit cell

Figure 4.1. (a) Schematic illustration of the acoustic barrier assembled from SCMs. It efficiently blocks the incoming sound waves $\left(p_{i}\right)$ indicated by the blue arrows. The substantially reflected $\left(p_{r}\right)$ and heavily suppressed transmitted $\left(p_{t}\right)$ sound fields are indicated by red and green arrows, respectively. (b) Sectional view of the cross-section of an SCM unit cell.

\subsection{Theoretical formulation of Fano-based metamaterials}

The Fano-based acoustic barrier consists of arrays of the SCMs, as shown in Figure 4.1(a). It is designed such that the incoming sound waves are efficiently blocked over a broad frequency range, achieving broadband high TL. The broadband nature of the acoustic barrier originates from multiple Fano resonances supported by the SCM coupling unit. As illustrated in Figure 4.1(b), the design of the SCM unit cell involves two identical ventilation channels located on the top and bottom of the coupling unit and a curled channel connecting the upstream and downstream with a space-coiling path. Space-coiling designs are usually used to generate linear resonances with optimal occupied space [47] and present an excellent capability to control the frequency distribution of not only fundamental but also higher-order modes $[93,94]$. In this dissertation, these strategies are employed to manipulate the local resonances and the resulting Fano resonances uniformly over the frequency region of interest. 
To study acoustic wave transmission and reflection behavior of the acoustic barrier, we introduce a simplified acoustic model of the SCM unit with the rigid side-wall boundary conditions shown in Figure 4.2 subjected to a normal incidence $p_{i}=e^{-i k x}$. In the simplified model, the actual curled channel is replaced with a straightened effective channel filled with effective fluid. The effective fluid is characterized by constitutive parameters $\left(\rho_{e}\right.$ and $\left.c_{e}\right)$ such that the weight of fluid inside the channel and sound travelling time in the two channels are guaranteed identical [8]. The ventilation channels are filled with air $\left(\rho_{0}\right.$ and $\left.c_{0}\right)$.

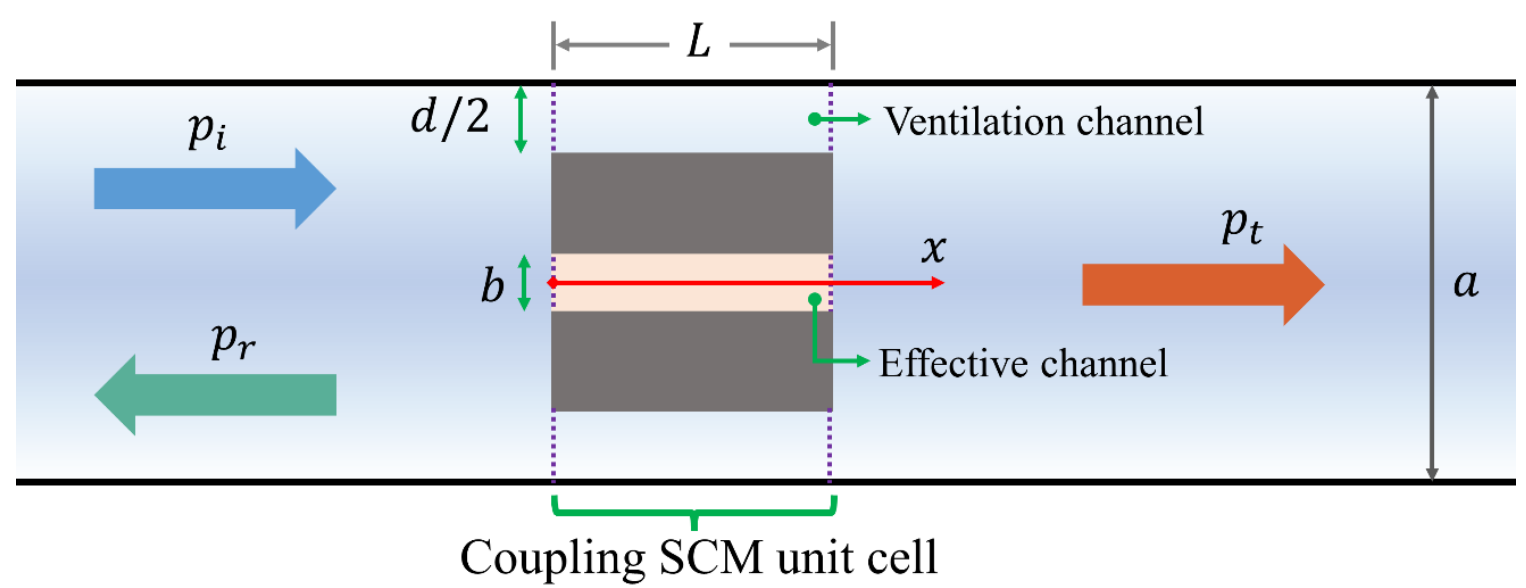

Figure 4.2. Schematic of an effective SCM coupling unit cell submerged in an impedance tube. $p_{i}, p_{r}$ and $p_{t}$ represent the incident, reflected and transmitted pressure fields, respectively.

The effective channel behaves as a linear resonator supporting both monopolar and dipolar local resonances [47, 91]. However, the ventilation channels only support resonances at higher frequencies and the acoustic pressure is linear in terms of phase change at lower frequencies. The transmission is the radiation from channels to the downstream, while the reflection is the combination of the radiation from the channels to the upstream and the reflection by the external hard wall of the coupling unit as $[58,59]$

$$
p_{t}(x, y)=p_{d} \text { and } p_{r}(x, y)=P_{i} e^{i k x}+p_{u}
$$


where $p_{u}$ and $p_{d}$ are the radiation fields in the upstream and downstream, respectively. They are related to the velocities at the inlet $(\mathrm{x}=0)$ and outlet $(\mathrm{x}=\mathrm{L})$ of the channels via Green's functions [65],

$$
\begin{aligned}
p_{u}(x, y)=-i \rho_{0} c_{0} k v_{e 1} \int_{b / 2}^{b / 2} G_{u}\left(x, y \mid 0, y_{0}\right) d y_{0} \\
-2 i \rho_{0} c_{0} k v_{\mathrm{v} 1} \int_{a / 2-d / 2}^{a / 2} G_{u}\left(x, y \mid 0, y_{0}\right) d y_{0},
\end{aligned}
$$

and

$$
\begin{aligned}
p_{d}(x, y)=-i \rho_{0} c_{0} k v_{e 2} \int_{\frac{b}{2}}^{\frac{b}{2}} G_{d}\left(x, y \mid L, y_{0}\right) d y_{0} \\
\quad-2 i \rho_{0} c_{0} k v_{\mathrm{v} 2} \int_{\frac{a}{2}-\frac{d}{2}}^{\frac{a}{2}} G_{d}\left(x, y \mid L, y_{0}\right) d y_{0} .
\end{aligned}
$$

Here, $\left(v_{e 1}, v_{e 2}\right)$ and $\left(v_{v 1}, v_{v 2}\right)$ are the velocities of the effective and ventilation channels at their inlet and outlet, respectively. $G_{u}\left(x, y \mid 0, y_{0}\right)$ and $G_{d}\left(x, y \mid L, y_{0}\right)$ are the Green's functions of the upstream $(x<0)$ and downstream $(x>L)$. They can be presented in terms of the mode shape functions of the waveguide [58, 95-97],

$$
G_{u}\left(x, y \mid 0, y_{0}\right)=\frac{1}{i k a}\left\{e^{i k x}+i \sum_{n=1}^{\infty} \frac{\varphi_{n}(y) \varphi_{n}\left(y_{0}\right)}{\alpha_{n}\left\langle\varphi_{n}^{2}(y)\right\rangle} e^{\alpha_{n} x}\right\}
$$

and 


$$
G_{d}\left(x, y \mid L, y_{0}\right)=\frac{1}{i k a}\left\{e^{-i k(x-L)}+i \sum_{n=1}^{\infty} \frac{\varphi_{n}(y) \varphi_{n}\left(y_{0}\right)}{\alpha_{n}\left\langle\varphi_{n}^{2}(y)\right\rangle} e^{-\alpha_{n}(x-L)}\right\}
$$

where $\varphi_{n}$ and $\alpha_{n}$ are the $n$ th-order mode shape function and corresponding eigenfrequency of the waveguide's cross-section, respectively.

$$
\varphi_{n}(y)=\cos \left(\frac{2 n \pi}{a} y\right), \quad \alpha_{n}=\sqrt{\left(\frac{2 n \pi}{a} \frac{c_{0}}{\omega}\right)^{2}-1} \text { and }\left\langle\varphi_{n}^{2}(y)\right\rangle=\frac{1}{a} \int_{-a / 2}^{a / 2} \varphi_{n}^{2}(y) d y
$$

Assuming that the effective and ventilation channels are narrow, the pressure fields inside them can be estimated by the fundamental mode [65] as

$$
p_{e}(x)=A_{e} e^{-i k_{e} x}+B_{e} e^{i k_{e}(x-L)}
$$

and

$$
p_{\mathrm{v}}(x)=A_{\mathrm{v}} e^{-i k x}+B_{\mathrm{v}} e^{i k(x-L)}
$$

respectively, where A and B are the amplitude coefficients by linearity in the channels and the wavevector of the effective channel $k_{e}=\omega / c_{e}$. The relations between the velocities and the pressures of the channels at their inlet and outlet are obtained by applying Euler's equation $\rho(\partial \vec{v} / \partial t)=-\nabla p$. Substituting equation (4.4) into equation (4.1) and equation (4.2) leads to the following set of equations in a matrix form for determining the normalized volume velocities at the inlet/outlet of the channels:

$$
\boldsymbol{M}\left[\begin{array}{l}
\bar{v}_{e 1} \\
\bar{v}_{e 2} \\
\bar{v}_{\mathrm{v} 1} \\
\bar{v}_{\mathrm{v} 2}
\end{array}\right]=\left[\begin{array}{l}
2 \\
0 \\
2 \\
0
\end{array}\right]
$$


where the normalized volume velocities $\bar{v}_{e 1}, \bar{v}_{e 2}, \bar{v}_{\mathrm{v} 1}$ and $\bar{v}_{\mathrm{v} 2}$ are defined by $\bar{v}=$ $\sigma \rho_{0} c_{0} v / P_{i}$ with $\sigma$ being the open ratio of the corresponding channels. $\boldsymbol{M}$ is a $4 \times 4$ matrix representing the coupling between the radiation fields of the channels. The elements of $\boldsymbol{M}$ are presented in equation (4.10).

$$
\begin{aligned}
& M_{11}=M_{22}=1-i\left(\frac{1}{\sigma_{e} \tan \alpha_{e}}-\delta_{e e}\right) \quad M_{12}=\frac{i}{\sigma_{e} \sin \alpha_{e}} \quad M_{13}=M_{24}=1+i \delta_{e v} \\
& M_{14}=M_{23}=0 \quad M_{33}=M_{44}=1-i\left(\frac{1}{\sigma_{\mathrm{v}} \tan \alpha_{\mathrm{v}}}-\delta_{\mathrm{vv}}\right) \quad M_{34}=\frac{i}{\sigma_{\mathrm{v}} \sin \alpha}
\end{aligned}
$$

Considering $|\mathrm{x}| \rightarrow \infty$, equation (4.1) and equation (4.2) result in the expressions of transmission and reflection in the far field,

$$
p_{t}(x)=P_{i}\left(\bar{v}_{e 2}+\bar{v}_{\mathrm{v} 2}\right) e^{-i k(x-L)}
$$

and

$$
p_{r}(x)=P_{i}\left(1-\bar{v}_{e 1}-\bar{v}_{\mathrm{v} 1}\right) e^{i k x},
$$

respectively. As a result, the transmission and reflection coefficients are readily obtained as

$$
T=\bar{v}_{e 2}+\bar{v}_{\mathrm{v} 2} \text { and } R=1-\bar{v}_{e 1}-\bar{v}_{\mathrm{v} 1}
$$

respectively. Now, we consider an effective homogeneous medium representing the coupling unit under the long-wavelength assumption. The effective medium is acoustically characterized by the effective mass density $\rho_{e f f}$ and the effective bulk modulus $\mathcal{B}_{e f f}$, which can be retrieved using the scattering matrix approach [98], 


$$
\left[\begin{array}{l}
P_{1} \\
U_{1}
\end{array}\right]=\left[\begin{array}{cc}
\cos \left(k_{e f f} a\right) & i Z_{\text {eff }} \sin \left(k_{\text {eff }} a\right) \\
i Z_{\text {eff }}^{-1} \sin \left(k_{\text {eff }} a\right) & \cos \left(k_{\text {eff }} a\right)
\end{array}\right]\left[\begin{array}{c}
P_{2} \\
U_{2}
\end{array}\right]
$$

where $P_{1}, U_{1}$ and $P_{2}, U_{2}$ are the total pressure and velocity fields evaluated on the left and right sides of the effective medium. Solutions to equation (4.9) lead to the effective velocity, impedance, mass density and bulk modulus, respectively, being

$$
\begin{aligned}
& c_{e f f}=\frac{\omega}{k_{e f f}}=\frac{\omega a}{\cos ^{-1}\left[\left(P_{1} U_{1}+P_{2} U_{2}\right) /\left(P_{1} U_{2}+P_{2} U_{1}\right)\right]}, \\
& Z_{e f f}=i \frac{P_{2}^{2}-P_{1}^{2}}{P_{1} U_{2}+P_{2} U_{1}} \sqrt{\frac{\left(P_{1} U_{2}+P_{2} U_{1}\right)^{2}}{\left(P_{2}^{2}-P_{1}^{2}\right)\left(U_{1}^{2}-U_{2}^{2}\right)^{\prime}}} \\
& \rho_{\text {eff }}=\frac{Z_{\text {eff }}}{c_{\text {eff }}} \text { and } B_{\text {eff }}=Z_{\text {eff }} c_{\text {eff }}
\end{aligned}
$$

To validate the above analysis, an effective unit cell with $\mathrm{a}=88.89 \mathrm{~mm}, \mathrm{~b}=11 \mathrm{~mm}, \mathrm{~d}=$ $26 \mathrm{~mm}, \mathrm{~L}=63 \mathrm{~mm}$ and $\rho_{e} / \rho_{0}=c_{0} / c_{e}=4.375$ is investigated theoretically and numerically. The numerical simulation is conducted using COMSOL Multiphysics. To investigate the Fano resonance involved and its associated sound reduction application, TL, defined as $20 \times \log _{10}\left|p_{i} / p_{t}\right|$, is assigned as the characterization measure of the sound reduction performance. Excellent agreement between the analytical and numerical TL spectra can be observed in Figure 4.3(a), proving the correctness of our theoretical model. As illustrated in Figure 4.3(a), two asymmetrical profiles of TL peaks and dips of the unit are clearly observed, similar to the Fano-like resonance. Note that the high TL region can be formulated between the two TL peaks for broadband sound mitigation if we design the unit cell properly. Those two asymmetric transmission profiles are due to the fact that the 
portion of the acoustic wave travelling through the resonating effective channel interferes with the portion of the acoustic wave travelling through the non-resonating ventilation channel. The asymmetric transmission profile, based on a Fano-like interference, possesses a peak region due to destructive interference resulting in attenuation of the transmitted wave.

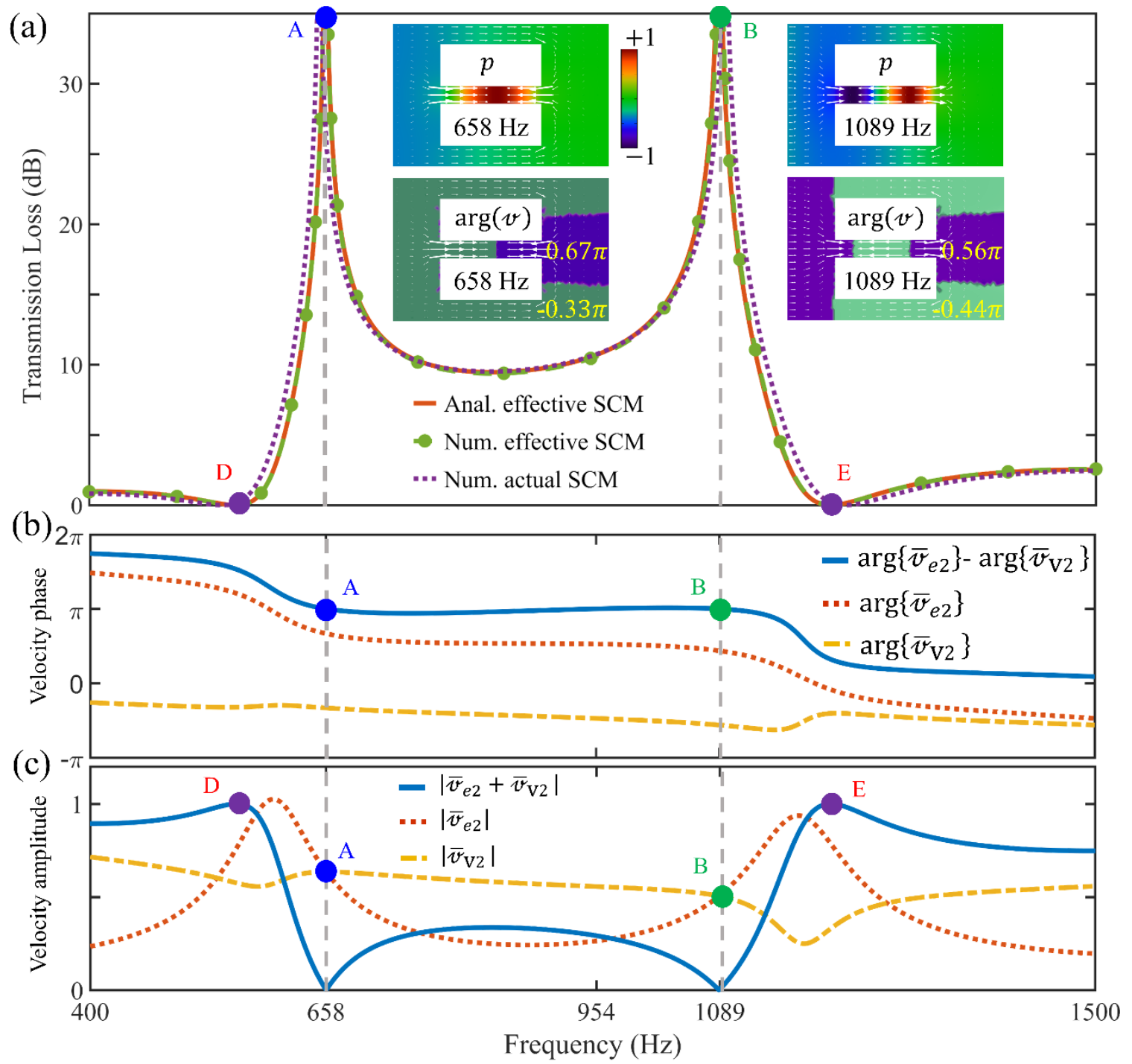

Figure 4.3. (a) TL spectra of the effective SCM unit cell. Theoretical and numerical results are shown, together with the numerical result of the actual SCM unit cell. At the two TL peaks, both the magnitude and phase of the velocity distributions of the effective SCM coupling unit are illustrated as insets. (b) Frequency-dependent phase spectra of the normalized volume velocities $\bar{v}$ at the outlet of the channels. (c) Frequency-dependent amplitude spectra of the normalized volume velocities $\bar{v}$ at the outlet of the channels. 
The TL peaks and dips, from another point of view, can be considered as the resonances and anti-resonances of the unit cell, respectively [58, 65]. The working mechanism of the unit cell at low frequencies can be explained by the weakly coupled effective and ventilation channels. For the interested frequency regime, the effective channel supports two local modes, i.e. monopolar and dipolar, evidenced by two TL dips at $560 \mathrm{~Hz}$ and 1121 $\mathrm{Hz}$, respectively, while the ventilation ones support an acoustic linear flow in terms of the phase change, evidenced by the smooth TL profile [see Figure 4.4].

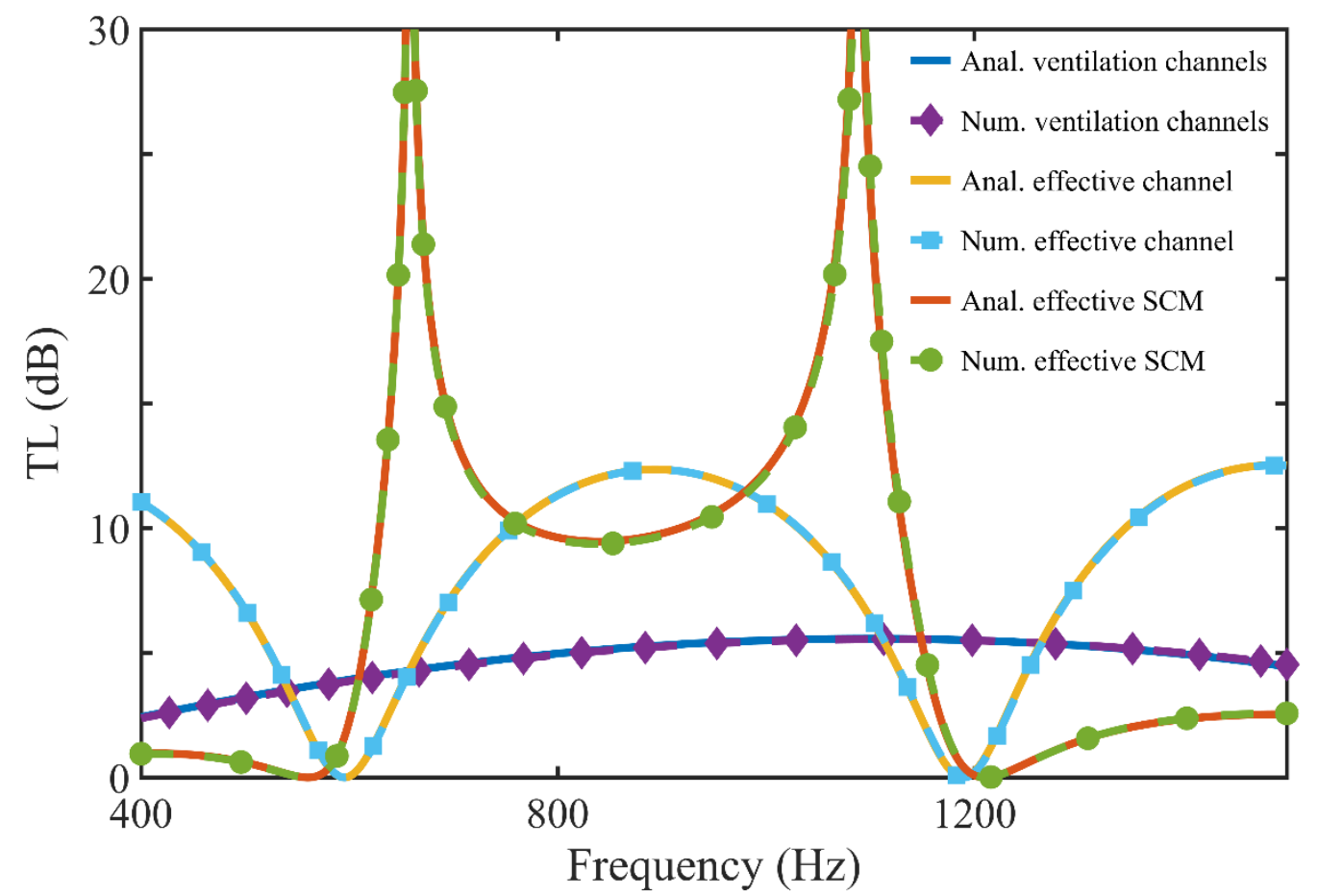

Figure 4.4. The transmission loss spectrum from analytical solution and numerical simulation in three cases: only ventilation channels, only effective channel and both of them presence on the SCM unit cell.

The coupling between these two channels induces resonance and anti-resonance in the unit cell. Since the former is much stronger than the latter, the induced resonance and antiresonance are located in the close vicinity of the local resonances of the effective channel. Therefore, the mode shape of the TL peaks and dips are mainly characterized by the mode shapes of the effective channel local resonances [see the pressure field $(\mathrm{p})$ described in the 
inset of Figure 4.3(a)]. It is noteworthy that the distributions of the resonances and antiresonances for the monopolar and dipolar modes are in the opposite order. Specifically, the monopolar mode at $600 \mathrm{~Hz}$ and dipolar one at $1121 \mathrm{~Hz}$ excite the resonances and antiresonances of the coupling unit at $560 \mathrm{~Hz}$ and $658 \mathrm{~Hz}$ and at $1175 \mathrm{~Hz}$ and $1089 \mathrm{~Hz}$, respectively, to realize a broadband TL spectrum.

To quantitatively interpret TL peaks and dips, we further plot the phase and magnitude of $\bar{v}$ at the two channel outlets in Figure 4.3(b) and Figure 4.3(c), respectively. According to equation (4.8), the occurrence of TL dips represents the condition $\bar{v}_{e 1}+\bar{v}_{\mathrm{v} 1}=1$ or $\bar{v}_{e 2}+$ $\bar{v}_{\mathrm{v} 2}=1$, meaning zero reflection. In other words, the total volume velocity transmitted through the inlets is equal to the incident volume velocity [see points D and E on Figure 4.3(c)], which obviously refers to the acoustic impedance matching condition. By contrast, the TL reaches a maximum when the transmission coefficient vanishes. The condition for TL peaks reads $\bar{v}_{e 2}+\bar{v}_{\mathrm{v} 2}=0$, which indicates that the normalized volume velocities at the outlets of ventilation and the effective channels are identical in amplitude, but are out of phase [see the phase distribution $(\arg (v)$ ] shown on the inset in Figure 4.3(a) and points A and B on Figure 4.3(b) and Figure 4.3(c)). In addition, the normalized acoustic admittance at the outlet of the effective coupling unit, $1 / Z_{2}=1 / Z_{e 2}+1 / Z_{v 2}=\bar{v}_{e 2} / p+$ $\bar{v}_{\mathrm{v} 2} / p$, is zero, indicating that the normalized acoustic impedance, $Z_{2} \rightarrow \infty$, and the outlet now are equivalently an acoustically rigid wall. In this case, the effective coupling unit blocks all the incoming pressure fields, leading to transmission zeros. Finally, as illustrated in Figure 4.3(b) and Figure 4.3(c), the phase difference between $\bar{v}_{e 2}$ and $\bar{v}_{\mathrm{v} 2}$ is nearly constant $(\pi)$ in the region between points A and B while their amplitudes show small difference. Therefore, high TL in the region between two peaks can be expected. 

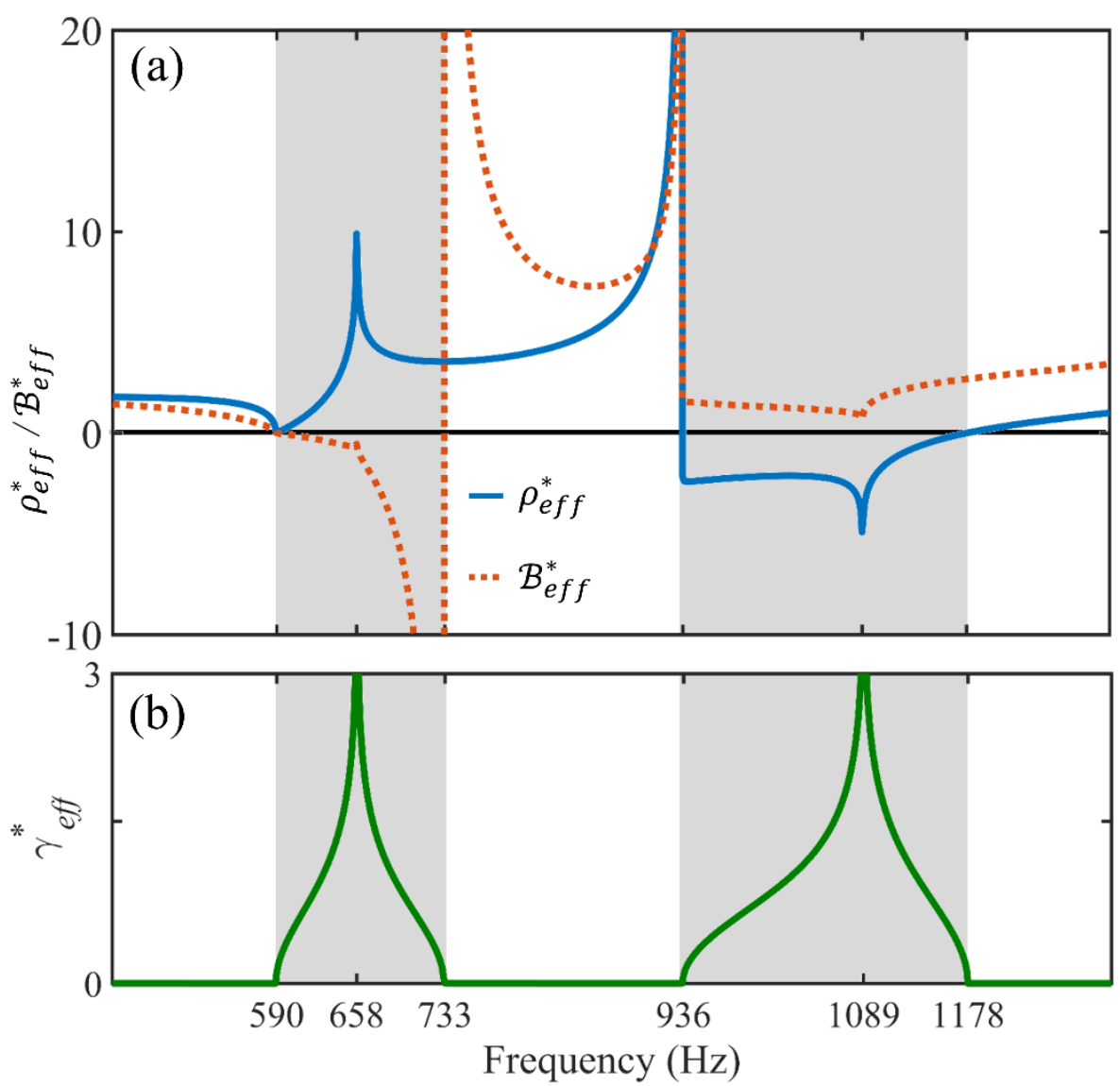

Figure 4.5. (a) Real part of the normalized effective mass density $\rho_{e f f}^{*}=\operatorname{Re}\left\{\rho_{e f f} / \rho_{0}\right\}$ and the bulk modulus $\mathcal{B}_{\text {eff }}^{*}=\operatorname{Re}\left\{\mathcal{B}_{\text {eff }} / \mathcal{B}_{0}\right\}$. (b) The decaying factor of the unit cell $\gamma_{\text {eff }}^{*}=-\operatorname{Im}\left\{k_{\text {eff }} a\right\}$. Two singlenegative bands are highlighted in grey.

The excellent sound reduction performance can also be explained using an effective medium. Following equation (4.11), we plot the real part of the normalized effective density $\rho_{\text {eff }}^{*}=\operatorname{Re}\left\{\rho_{\text {eff }} / \rho_{0}\right\}$ and bulk modulus $\mathcal{B}_{\text {eff }}^{*}=\operatorname{Re}\left\{\mathcal{B}_{\text {eff }} / \mathcal{B}_{0}\right\}$ in Figure 4.5(a). Two separate single-negative stop bands (SNBs), i.e. a negative density region (590-733 $\mathrm{Hz})$ and a negative bulk modulus region $(936-1178 \mathrm{~Hz})$, can be seen. The negative bulk modulus is responsible for the first Fano resonances with the monopolar mode and the negative density can be used to capture the second Fano resonances with the dipolar mode. The normalized decaying factor (NDF), defined as $\gamma_{e f f}^{*}=\operatorname{Im}\left\{-k_{e f f} a\right\}$, is presented in Figure 4.5(b). It can be observed that $\gamma_{\text {eff }}^{*}$ is zero everywhere except within the SNBs, 
whereas $\operatorname{Re}\left\{k_{\text {eff }}\right\}$ (not shown here), which represents the propagating part of the sound wave, is zero within SNBs and positive at other frequencies. Within the SNB regions, the NDF takes large values and reaches a maximum at the Fano resonances, meaning that the wave amplitude exponentially decays. In brief, the two Fano resonances result in two SNBs providing considerably high TL, efficiently blocking the sound field.

\subsection{Dampening space coiling metamaterial}

Although the combination of two symmetrical Fano-like profiles leads to a broadband TL spectrum, two dips corresponding to two coupling resonances of the coupling unit still need to be fixed to optimize the performance of an acoustic barrier built from an array of coupling units. For real applications, which often require an ultra-broadband working region, the previously achieved TL profile is not optimal. Weakening the local resonances gives weaker Fano resonances, lifting the TL dips but inevitably lowering the peaks simultaneously. This process results in a more practically optimal TL profile. Dampening the SCM is the most straightforward way to weaken the local resonances. In theory, this can be achieved by considering the intrinsic thermal and external acoustic viscosity.

\subsubsection{Thermal viscosity}

In real structures, there always exists thermal viscosity inside the viscous boundary layers. Its effect is non-negligible around the resonances $[6,13]$ when the induced velocity is large, especially within the SCM where the friction area is massive. The intrinsic thermal viscosity may help to lift the dips of the Fano TL profile, ensuring optimized TL performance within the range of interest. To take into account the thermal viscosity, the density and sound velocity inside the SCM should be modified as [99] 


$$
\rho_{v}=\frac{\rho_{0}}{\psi_{v}} \text { and } \quad c_{v}=c \sqrt{\frac{\psi_{v}}{\gamma-(\gamma-1) \psi_{h}}}
$$

respectively, where $\psi_{v}$ and $\psi_{h}$ are the viscosity-geometry and thermal-geometry functions for slits,

$$
\psi_{v}=1-\frac{\tan \left(k_{v} b / 2\right)}{k_{v} b / 2} \text { and } \psi_{h}=1-\frac{\tan \left(k_{h} b / 2\right)}{k_{h} b / 2} \text {, }
$$

Here, the viscous and thermal lengths are $k_{v}=\sqrt{-i \rho_{0} \omega / \eta}$ and $k_{h}=\sqrt{-i \rho_{0} \omega C_{p} / k}$, respectively, with $\eta$ being the dynamic viscosity and $C_{p}$ the heat capacity at constant pressure of air. The thermal viscous effect can be observed in the TL spectra of two cases: the SCMs without (lossless, blue in Figure 4.6(d)) and with viscosity (viscous, red curve in Figure 4.6(d)) applied in the curled channel. They are identical within most regions except around the resonances, as predicted. At the Fano resonances, instead of reaching $\infty$ in theory, thermal viscosity dampens the resonances, making the peaks less sharp with the maximum TL decreased to less than $35 \mathrm{~dB}$. By contrast, the TL dips are lifted slightly. The thermal viscous effect of these regions can be enhanced using narrower channels with a smaller open ratio $b / a$. However, narrower channels are usually adverse to improving the TL performance [36]. For practical noise reduction applications, the TL dips should be lifted by using a stronger dampening mechanism. 


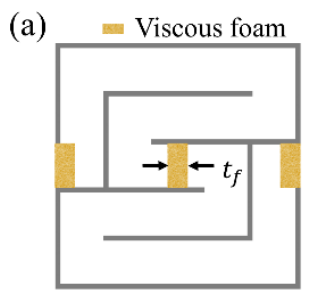

(b) - Monopole "- Dipole

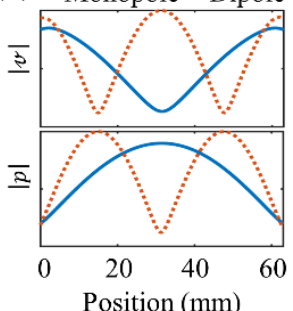

(c)

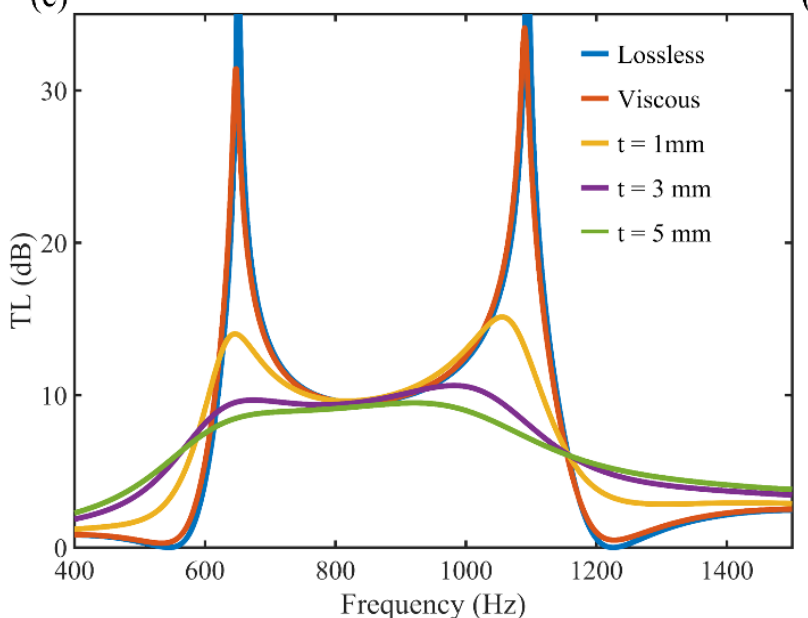

(d)

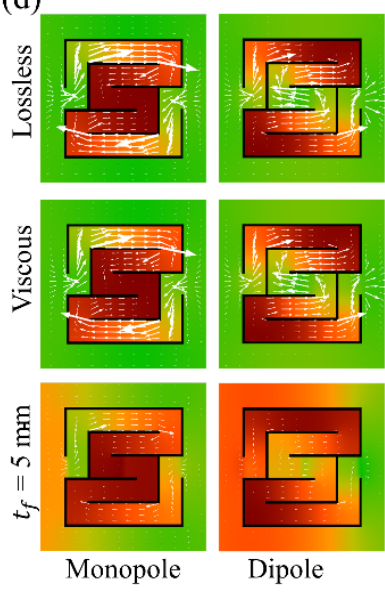

Figure 4.6. (a) Diagram of the deployment of viscous foams. All foam layers have identical thickness $t_{f}$.

(b) Velocity and pressure profiles along the effective channel at its monopolar and dipolar modes. (c) TL spectra of the actual SCM with different configurations of the space-coiling channel: without thermal viscosity, with thermal viscosity and with dampening foam layers of thicknesses $1 \mathrm{~mm}, 3 \mathrm{~mm}$ and $5 \mathrm{~mm}$.

(d) The pressure and velocity fields at the monopole and dipole in three cases: lossless, viscous and $5 \mathrm{~mm}$ foam employed. The velocity representing the strength of the resonances is heavily dampened in the case of viscous foam employed, so it is called 'acoustic hyper-damping'.

\subsubsection{Acoustic hyper-damping}

In this dissertation, acoustic hyper-damping is introduced by deploying acoustic foam layers at the velocity anti-nodes of the SCM, as shown in Figure 4.6(a). Figure 4.6(b) exhibits the velocity and pressure profiles, which are opposite to each other in space. The acoustic foam is an open-cell porous material, comprising two phases, i.e. an elastic matrix (solid phase) phase and a fluid phase filling the elastic matrix. The porous matrix gives a large friction area (contacting surface) between the two phases. Therefore, when submerged into a high fluid velocity region, it is expected to absorb a substantial amount of fluid flow energy. The well-known Biot model $[100,101]$ is the most comprehensive model for describing the vibro-acoustics of the coupling between the two phases. It requires several experimental inputs, and the corresponding calculation is quite time consuming. For this reason, the effective medium theory is used to model equivalently the porous acoustic material. Among all the existing models, the five-parameter model proposed by 
Johnson-Champoux-Allard (JCA) is the most widely used as it provides simplicity and high accuracy. In the JCA model, the mass density characterizing the visco-inertial effects was proposed by Johnson et al. [102] as

$$
\tilde{\rho}(\omega)=\frac{\tau_{\infty} \rho}{\varepsilon_{p}}\left[1+\frac{\varepsilon_{p} \phi_{f}}{i \omega \rho \tau_{\infty}} \sqrt{1+i \frac{4 \rho \omega \eta \tau_{\infty}^{2}}{\varepsilon_{p}^{2} \phi_{f}^{2} \Lambda_{v}^{2}}}\right]
$$

and the bulk modulus is [103]

$$
\widetilde{\mathcal{B}}(\omega)=\frac{\gamma P_{0} / \phi_{f}}{\gamma-(\gamma-1)\left[1-i \frac{8 k}{\rho \omega C_{p} \Lambda_{t}^{2}} \sqrt{\left.1+i \frac{\rho \omega C_{p} \Lambda_{t}^{2}}{16 k}\right]^{-1}}\right.}
$$

where $P_{0}$ and $k_{d}$ denote the fluid equilibrium pressure and thermal conductivity of the fluid phase. The five parameters of the JCA model, $\varepsilon, \phi_{f}, \Lambda_{v}, \Lambda_{t}$ and $\tau_{\infty}$, used to describe the acoustic foams are estimated by fitting the absorption of a pure foam layer, backed by a rigid wall under normal incidence in simulations and experiments [see Figure 4.7 for the setup and measurement result of absorption of acoustic foam]. The optimal values are listed in Table 4.1.

Table 4.1. Porous matrix properties of the acoustic foam

\begin{tabular}{lcc}
\hline Properties & Symbol & Value \\
\hline Porosity & $\varepsilon_{p}$ & $96 \%$ \\
Flow resistivity & $\phi_{f}$ & $32000 \mathrm{~N} \cdot \mathrm{s} / \mathrm{m}^{4}$ \\
Viscous characteristic length & $\Lambda_{v}$ & $78 \mu \mathrm{m}$ \\
Thermal characteristic length & $\Lambda_{t}$ & $119 \mu \mathrm{m}$ \\
Tortuosity factor & $\tau_{\infty}$ & 1.83 \\
\hline
\end{tabular}




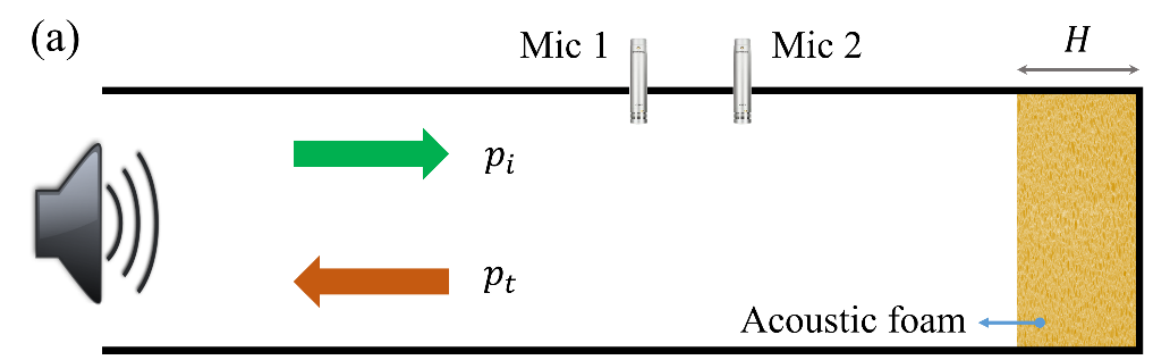

(b)

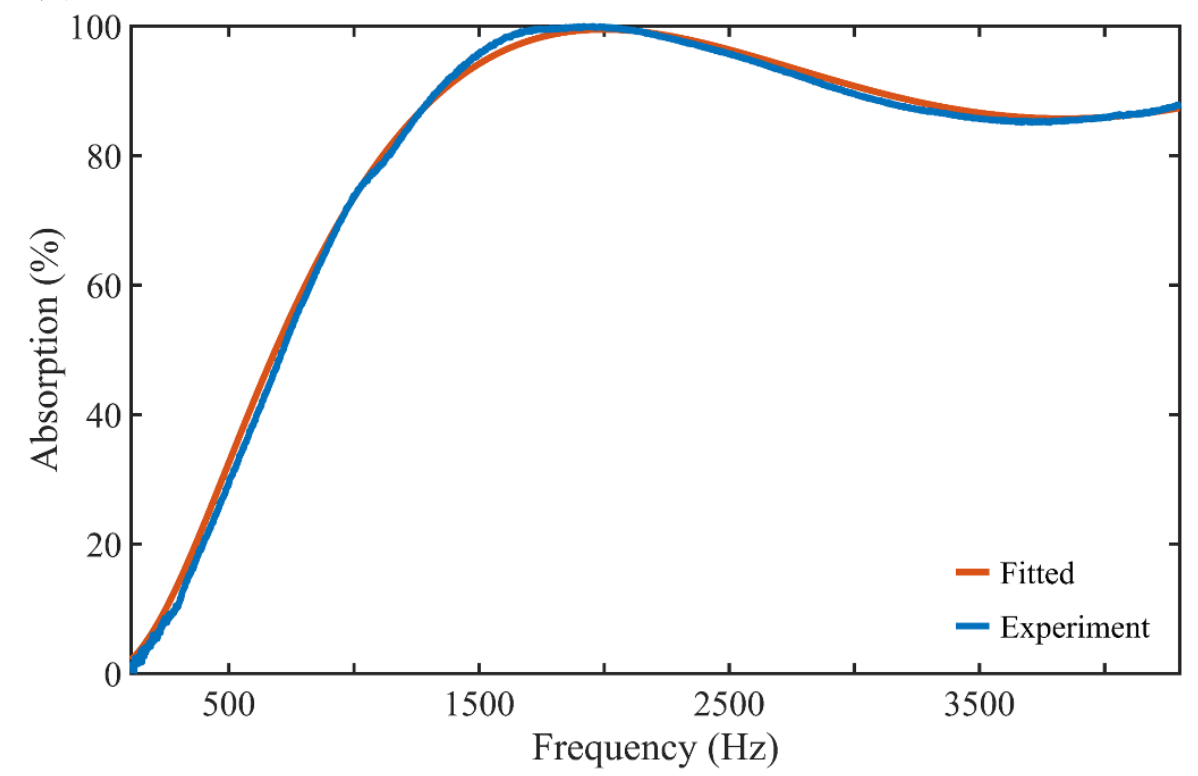

Figure 4.7. (a) The setup of experiment to determine the effective parameter of acoustic foam. An impedance tube with two condense microphones are used to measure the absorption of the foam. (b) The absorption from experiment and fitting model.

The TL spectra for three foam layer thicknesses, $1 \mathrm{~mm}, 3 \mathrm{~mm}$ and $5 \mathrm{~mm}$, are presented in Figure 4.6(c). It is found that the local resonances of the SCM are heavily dampened. Even with the foam layers of only $1 \mathrm{~mm}$ thickness, the TL dips are completely removed, giving rise to the optimized TL profile. This is exactly the function of hyper-damping in the SCM. The evidence of hyper-damping can also be recognized through the velocity field inside the heavily dampened coiling channel (Figure 4.6(d)), which represents the strength of its resonances. Since the relation between TL dips and peaks is causality, lifting the dips means dampening the local resonance of the SCM, which inevitably dampens the TL peaks as well. The foam thickness plays an important role in optimizing the TL profile. Increasing 
the foam thickness $t_{f}$ leads to a relatively more uniform TL profile. When $t_{f}=5 \mathrm{~mm}$, the peaks and dips are nearly unrecognizable because the SCM is over-dampened. Further increasing the foam thickness will eventually make the TL profile approach the case where the unit cell includes only ventilation channels [see Figure 4.4]. This is simply because the foam filling the effective channel results in high impedance, driving the channel to perform as a solid medium rather than as a linear acoustic channel.

\subsection{Application in ultra-broadband sound insulation}

We employ the SCMs to construct an acoustic barrier possessing efficient ventilation, sound isolation and absorption functions across an ultra-broadband $0.44-0.385 \mathrm{kHz}$. This range completely covers nine third-octave bands $(0.447-3.548 \mathrm{kHz})$, including the frequency spectrum of most typical airborne noise in living and working environments. The target frequency range is selected such that the metamaterial barrier can work well within the stable region of the impedance tube since the cut-off frequency of the tube is around $3.85 \mathrm{kHz}$. The proposed barrier, as shown in Figure 4.8(a), consists of the supercell bars detailed in Figure 4.8(b). The super-cell bar is only $220 \mathrm{~mm}$ thick $\left(t_{\text {bar }}=220\right.$ $\mathrm{mm}$ ), and comprises three SCMs with the same open ratio, i.e. $\sigma_{e}=1-L / a=29.25 \%$, but different curled channel widths $(5.9 \mathrm{~mm}, 7.9 \mathrm{~mm}$ and $11 \mathrm{~mm})$. The super-cell bars without and with the dampening foam layers are considered, as shown in Figure 4.8(b). As presented in Figure 4.8(c), under normal incidence, the TL spectra of individual SCMs [see the blue, red and orange curves] show Fano profiles of not only fundamental but also higher-order resonances. They are distributed nearly uniformly over the frequency range of interest. The barrier consisting of all three SCMs under normal incident sound therefore shows satisfactory sound reduction performance [see the purple curve] within the broad 
region $0.3-4 \mathrm{kHz}$. However, there still exists many sharp TL dips due to the local resonances of the SCMs and the Fabry-Pérot resonances between the SCMs. To get rid of these TL dips to make the high-performance band (overall $>10 \mathrm{~dB}$ ) broader and smoother, $5 \mathrm{~mm}$ thick foam layers are employed to introduce the acoustic hyper-damping in the acoustic barrier. The simulation result is highlighted by the green curve in Figure 4.8(c). Significantly better overall performance with TL dips dampened or removed is shown, although most of the TL peaks are dampened as well and even disappear. It is worth mentioning that some TL dips are even converted into peaks (at $1234 \mathrm{~Hz}$ corresponding to the monopolar mode of the second SCM and at $1590 \mathrm{~Hz}$ corresponding to the monopolar mode of the first SCM). In fact, noise usually covers a broad range of incident angle. Therefore, we also numerically investigate the angular dependence of this barrier, and show that very good sound insulation performance can be found even for a large oblique angle of incidence [see Figure 4.9].

(a)

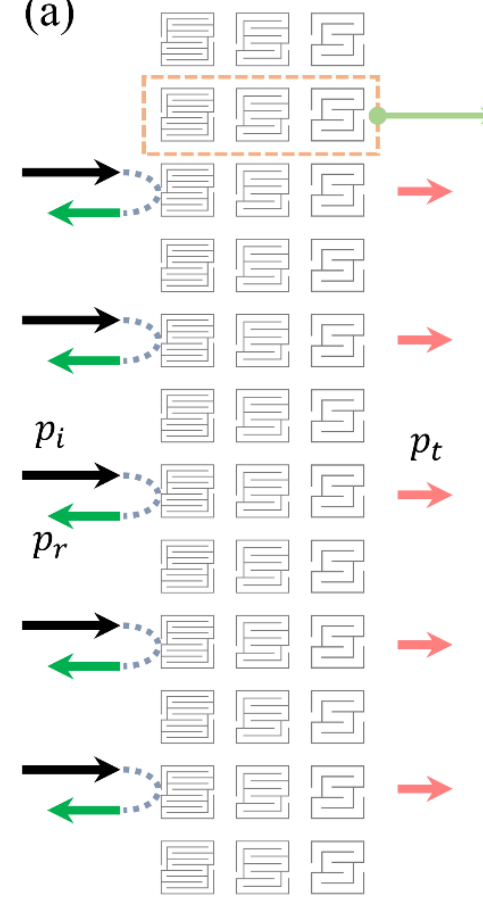

(b)

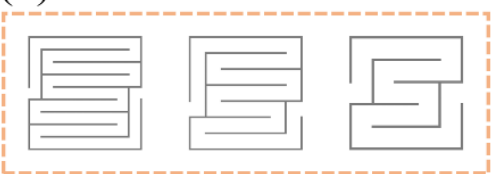

Undampened Barrier

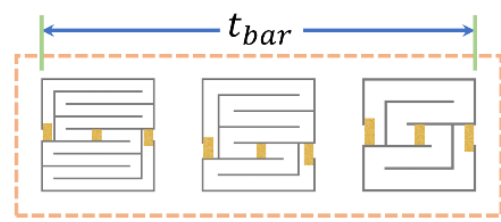

Dampened Barrier (c) $-\infty 1^{\text {st }}$ cell $--2^{\text {nd }}$ cell $\rightarrow 3^{\text {rd }}$ cell - Bar $\cdots$ Damp Bar

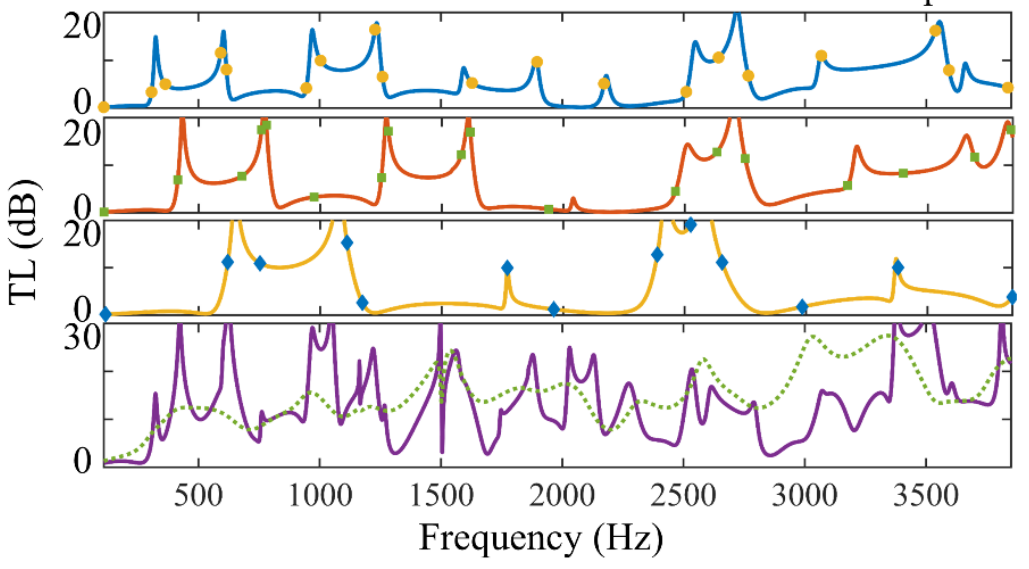


Figure 4.8. (a) Schematic of the acoustic barrier wall comprising three space-coiling cells with the same open ratio but different channel widths. (b) Zoom-in views of the super-cell bar without and with the dampening foam layers. (c) Numerically obtained transmission loss spectra of the lossless individual unit cells (blue, red and yellow curves with symbols), lossless super-cell (purple solid) and dampened super-cell (green dashed).

(a)

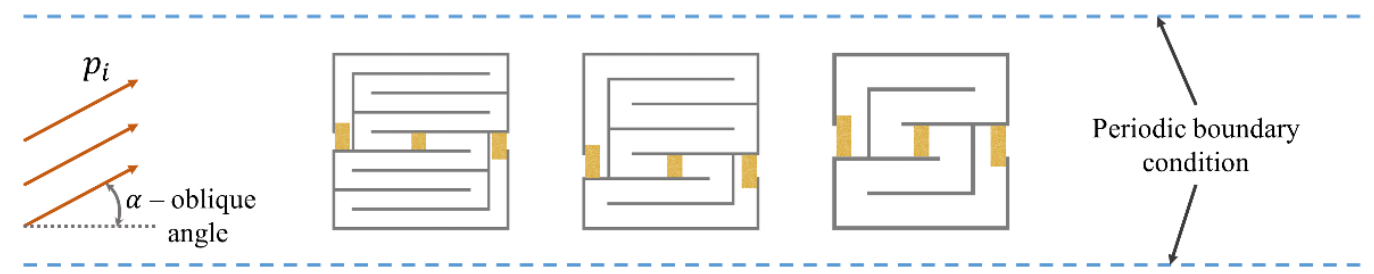

(b)

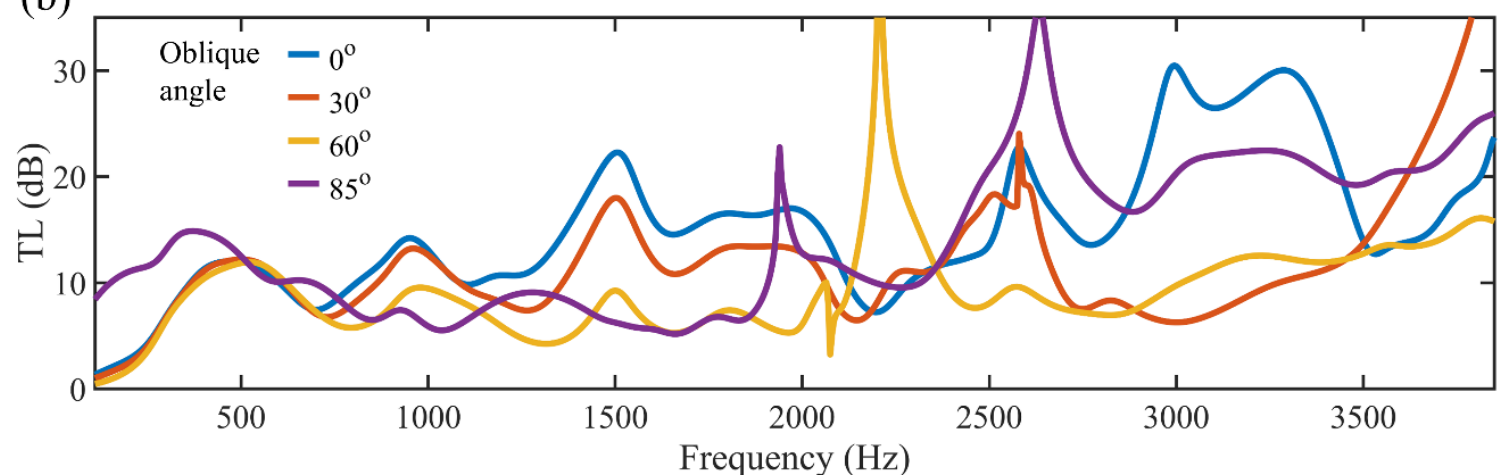

Figure 4.9. (a) Schematic illustration of the simulation model under oblique incidence. Top and bottom of the ribbon are defined by Floquet periodic boundary conditions. (b) Transmission loss (TL) spectra of the barrier at various angles of incidence.

To verify the proposed design and the simulation results, a super-cell sample is fabricated using a 3D printer. The sample containing acoustic foams, installed in the impedance tube, is shown in the inset of Figure 4.10(a). The performance of the super-cell, which equivalently represents the barrier's performance under normal incidence, is then evaluated in an impedance tube, as illustrated in Figure 4.10(a). The experimental results for the dampened acoustic barrier [see the red curves] are presented to make a comparison with the simulation results [see the blue curves] in the top panel of Figure 4.10(b). Good agreement is observed. The experiment exhibits ultra-broadband sound reduction performance within the range $0.3-3.85 \mathrm{kHz}$. The dampened design exhibits the TL of at least $10 \mathrm{~dB}$ within $0.44-3.85 \mathrm{kHz}$ covering completely nine third-octave bands, with an average $\mathrm{TL}$ of $17.7 \mathrm{~dB}$. In addition to the $\mathrm{TL}$, sound absorption of the dampened barrier 
defined as $A=1-|R|^{2}-|T|^{2}$ is also experimentally studied. Presented in the bottom panel of Figure 4.10(b) is the absorption of the dampened barrier under normal incidence. Overall, the simulation (blue curve) and experimental (red curve) results match well. The averaged absorption within the range $0.44-3.85 \mathrm{kHz}$ is $65 \%$. Several high-absorption bands are recognized experimentally: over $80 \%$ within the range $0.3-0.41 \mathrm{kHz}$ and near $100 \%$ at $340 \mathrm{~Hz}, 490 \mathrm{~Hz}, 2965 \mathrm{~Hz}$ and $3810 \mathrm{~Hz}$. After all, the proposed barrier, together with the acoustic hyper-damping, not only achieves ultra-broadband sound reduction while preserving ventilation efficiency but also exhibits excellent absorption capability.

(a)

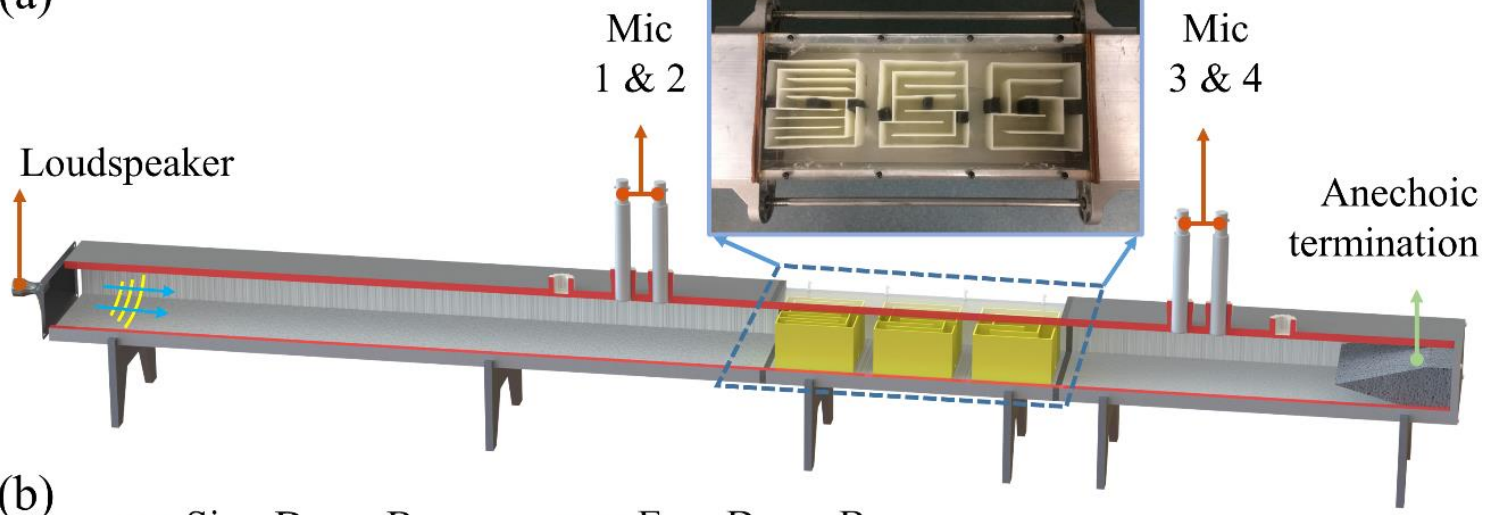

(b)
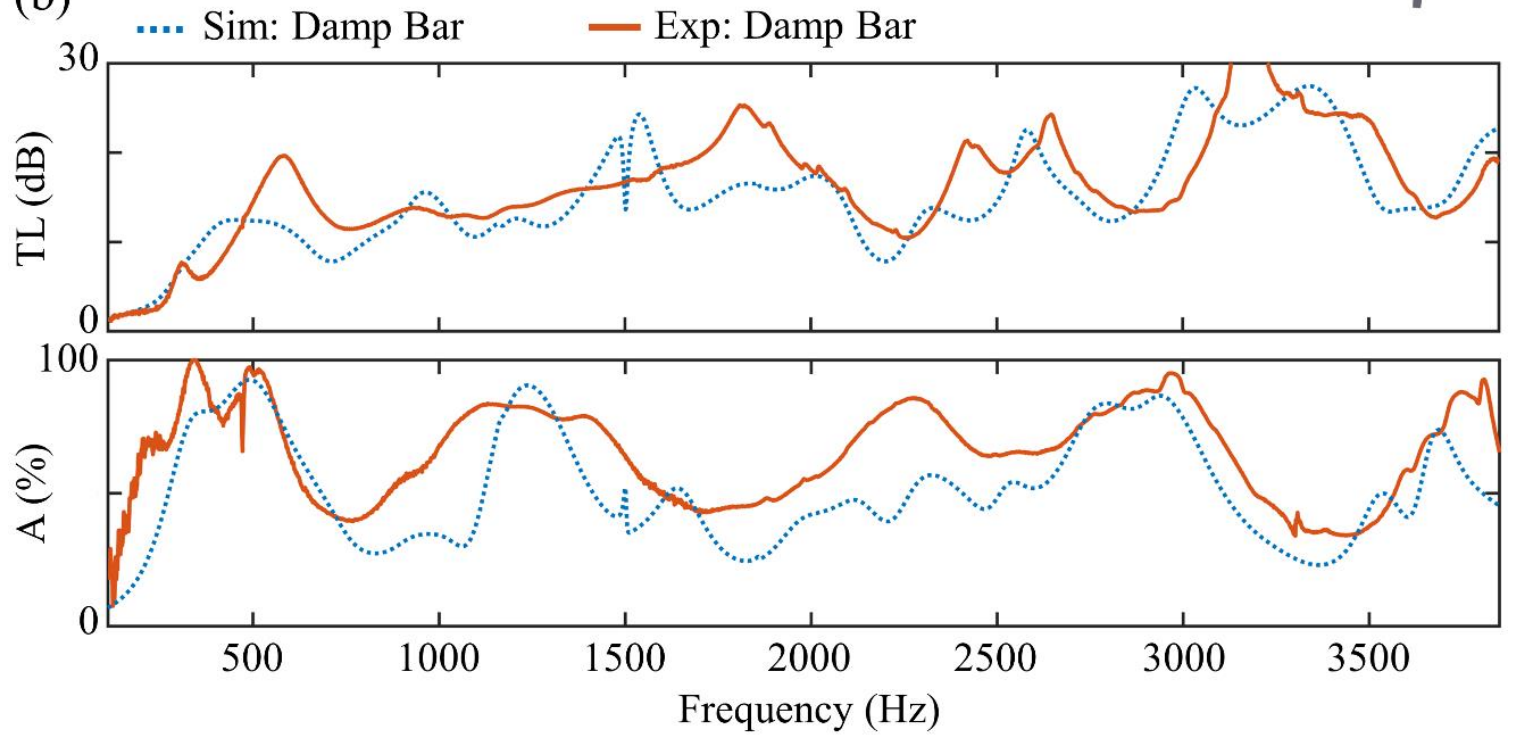

Figure 4.10. (a) Sectional view of the impedance tube and the samples in the experiment. The inset shows the detailed view of the dampened super-cell. (b) Comparison of the transmission loss (TL) and absorption (A) spectra between simulation and experiment results for the dampened super-cell. 


\subsection{Conclusion}

We have studied, analytically, numerically and experimentally, SCMs supporting two types of Fano resonances for the realization of ultra-broadband sound reduction application. The proposed SCMs support both monopolar and dipolar modes which interact with the background continuum mode and eventually produce acoustic Fano resonances. The resultant Fano resonances are beneficial in producing ultra-broadband TL. The acoustic barrier with a thickness of $220 \mathrm{~mm}$ and an open ratio of $29.25 \%$, derived from deploying periodically super-cells of three different SCM cells, shows satisfactory sound reduction performance within the broad frequency region $0.44-3.85 \mathrm{kHz}$, with the help of acoustic hyper-damping. Moreover, the acoustic absorption of the dampened barrier is measured to be overall beyond $65 \%$ with several perfect absorption regions. We believe this work paves the way for realizing effective broadband sound insulation and sound wave control devices. 


\section{Chapter 5. Helmholtz Resonator Based Acoustic Silencing}

\subsection{Introduction}

Conventional airborne sound attenuation essential in acoustic barriers refers to either reflecting or absorbing the incident acoustic energy while blocking airflow transport. The modern demand for sound attenuation, while preserving ventilation, such as the applications of mitigating engine and office noise, has prompted considerable effort put to the research of duct acoustics [73, 104-106]. Among the existing works, Herschel-Quincke waveguides [107-109] are notable, as the narrowband sound attenuation can be achieved with minimal reduction in the duct airflow area. Although these classical designs indeed provide simultaneous sound attenuation and ventilation, their inherent induct nature, along with their large physical footprint, limits the versatility and practical implementation. In practice, a more winding lined path supports a more prominent noise reduction, which in turn results in a larger pressure drop and a poorer ventilation effect. Hence, there has always been a trade-off between the efficiency of ventilation and the noise reduction. Acoustic local resonators have been widely studied in various noise control environments for lowfrequency noise reduction. Typical applications include sound diffusers and absorbers in room acoustics $[49,86,110,111]$, improvement of sound transmission loss (TL) of aircraft sidewalls $[1,55,112]$, acoustic metamaterials, and so forth. Among them, purely acoustic resonators like Helmholtz and Fabry-Perot resonators are most employed due to their high Q-factors [105] and simple structures. Recently, acoustic local resonators were also utilized as side branches in ducts or pipes in order to mitigate the sound transmission inside waveguides $[9,11,91]$. However, strong TL across broadband is still difficult to be 
achieved in the subwavelength regime, owing to the existence of strong antiresonances [8, 9, 13]. Meanwhile, the restriction on the gradient length of the coiled channel resonators often leads to practical challenges in assembling multiple functional units for the realization of broadband absorption and attenuation [11, 91, 93, 113]. Moreover, in the acoustic barrier designs $[11,12,91]$, the local acoustic resonators are immersed in the airflow to achieve high transmission loss. However, the smaller working cross-sectional area and discontinuous airflow inevitably result in a lower ventilation efficiency.

To address the aforementioned issues, we theoretically investigate and experimentally validate a subwavelength double-layer acoustic silencer (DAS) composed of multiple slittype Helmholtz resonators (SHRs), possessing subwavelength slit necks and enabling broadband TL and effective air ventilation. The DAS is constructed with two compactly assembled single-layer acoustic silencers (SASs) to enhance the broadband sound insulation performance. Such an acoustic barrier effectively blocks over $99.9 \%$ of the incident sound energy over a broadband frequency range of $0.48-0.95 \mathrm{kHz}$ while maintaining excellent ventilation due to the straight and conserved airflow cross section. In the theoretical part, we derive a comprehensive analytical model for the individual SHR, together with its derivatives, namely, the SAS and the DAS, to gain better understanding of the involved physical mechanism. The corresponding numerical and experimental results effectively support the developed theory with excellent agreement between them. Finally, we strongly believe that our work uncovers the fundamental characteristics of the slit-type Helmholtz resonators and may pave the way for designing highly efficient subwavelength acoustic silencers with broadband operation and great tunability 


\subsection{Slit-type Helmholtz resonator}

We start by designing a slit-type Helmholtz resonator (SHR), which serves as the building block of the compact acoustic silencer. Figure 5.1(a) provides the sectional view of the three-dimensional (3D) model of a side-branched SHR. The SHR is axisymmetric and consists of a cavity and a slit neck. Due to the massive friction area inside the neck, the dissipated energy via the thermal viscosity is expected to be significantly amplified. Both the resonance frequency of the SHR and the thermal viscosity can be tuned flexibly by varying the slit thickness and the cavity depth. Figure 5.1(b) illustrates the schematic of a single SHR with all the geometric parameters denoted for the complete theoretical analysis. Due to the complex geometry of the SHR, in the theoretical modeling, we divide the SHR into four domains (cavity A and necks B, C, and D). The acoustic impedance of the SHR is derived using a backtracking procedure: $\mathcal{S}_{a} \rightarrow \mathcal{S}_{b} \rightarrow \mathcal{S}_{c} \rightarrow \mathcal{S}_{d}$. To keep the equation representation simple, let us employ some denotations during the theoretical analyzing as listed in the Table 5.1.

(a)

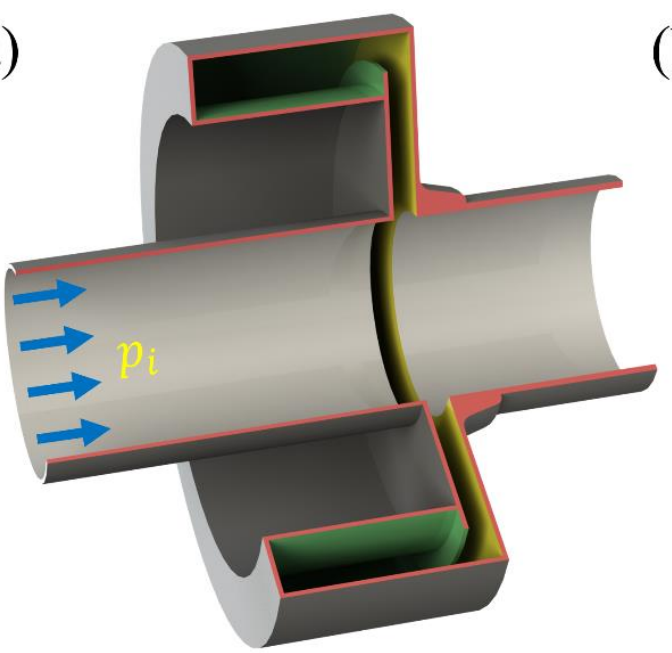

(b)

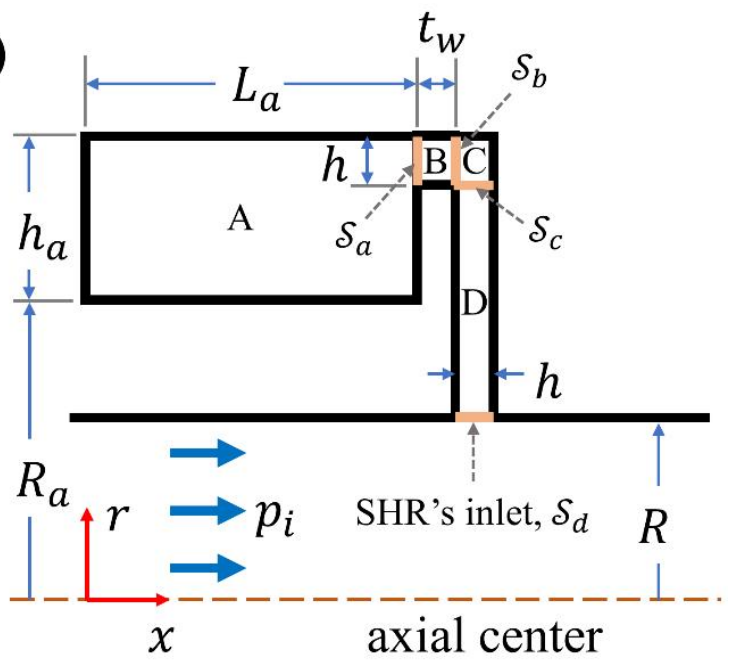

Figure 5.1. (a) Sectional view of a side-branched SHR. The blue arrows correspond to the incoming acoustic waves. (b) Schematic of a SHR with all the involved geometrical parameters indicated. The SHR is divided into multiple domains, labeled as A, B, C, and D. 
Table 5.1. Denotations on theoretical analysis.

Symbol Meaning

$\mathcal{S}_{x}$

The inlet area of the domain $\mathrm{X}$; here $\mathrm{X}$ can be $\mathrm{A}, \mathrm{B}, \mathrm{C}$ and $\mathrm{D}$

$\mathcal{S}_{v}$ and $\mathcal{S}_{0}$

The cross-sectional area of the cavity A and the duct

\langle\rangle$_{x}$

The average over the $\mathcal{S}_{x}$

\langle\rangle$_{x y}$

The average over the $\delta_{x}$ and then over the $\delta_{y}$

\langle\rangle$_{X}$

The average over the domain $\mathrm{X}$

$\bar{v}_{x}$

Average normal velocity over the $\mathcal{S}_{x}$

$\bar{p}_{x}$

Average pressure over $\mathcal{S}_{x}$

$\rho_{x}, c_{x}$ and $k_{x} \quad$ The mass density, sound velocity and wavenumber in the domain $\mathrm{X}$

We start with domain A. Since only the axisymmetric mode can be excited, the pressure and velocity fields inside domain A can be expressed, respectively, as

$$
\begin{aligned}
& p_{a}=\sum_{n=0}^{\infty} A_{n} \psi_{n}(r) \cos \left(\gamma_{x n} x\right) \text { and } \\
& v_{a}=\frac{i}{k_{a} \rho_{a} c_{a}} \frac{\partial p_{a}}{\partial x}=-\frac{i}{\rho_{a} c_{a}} \sum_{n=0}^{\infty} A_{n} \frac{\gamma_{x n}}{k_{a}} \psi_{n}(r) \sin \left(\gamma_{x n} x\right)
\end{aligned}
$$

where $\psi_{n}(r)$ represents the $n$-the order mode shape,

$$
\psi_{n}(r)=J_{0}\left(\gamma_{r n} r\right)-\alpha_{a n} Y_{0}\left(\gamma_{r n} r\right)
$$


The radial, $x$-directional wavenumbers $\gamma_{r n}$ and $\gamma_{x n}$, and $\alpha_{a n}$ are determined through the sound hard boundary conditions at the inner and outer shells of cavity A

$$
\begin{aligned}
& \alpha_{a n}=\frac{J_{1}\left(\gamma_{r n} R_{a}\right)}{Y_{1}\left(\gamma_{r n} R_{a}\right)}=\frac{J_{1}\left(\gamma_{r n}\left(R_{a}+h_{a}\right)\right)}{Y_{1}\left(\gamma_{r n}\left(R_{a}+h_{a}\right)\right)} \text { and } \\
& \gamma_{x n}^{2}=\left(\frac{\omega}{c_{a}}\right)^{2}-\gamma_{r n}^{2}
\end{aligned}
$$

Here, $J_{n}$ and $Y_{n}$ are the $n$-th order Bessel functions of the first and second kind, respectively. Employing the orthogonal property of the wavefunction $\psi_{n}(r)$, the coefficient of the wavefunction $A_{n}$ can be determined as below

$$
A_{n}=i \rho c \frac{\mathcal{S}_{a}}{\mathcal{S}_{v}} \frac{k}{\gamma_{x n}} \frac{\left\langle\psi_{n}\right\rangle_{a}}{\left\langle\psi_{n}^{2}\right\rangle_{v}} \frac{\bar{v}_{a}}{\sin \left(\gamma_{x n} L_{a}\right)}
$$

Plugging equation (5.4) back into equation (5.1), one can derive the specific acoustic impedance at $\mathcal{S}_{a}$ as

$$
Z_{a}=\frac{\bar{p}_{a}}{\bar{v}_{a}}=i \rho_{a} c_{a} \frac{\mathcal{S}_{a}}{\mathcal{S}_{v}} \beta
$$

Defining the normalized wavenumber in the domain A, $\bar{\gamma}=\gamma / k_{a}$, the normalized acoustic impedance of the cavity A takes the following form.

$$
\beta=\cot \left(k_{a} L_{a}\right)-\sum_{n=1}^{\infty} \frac{\operatorname{coth}\left(k L_{a} \sqrt{\bar{\gamma}_{r n}^{2}-1}\right)}{\sqrt{\bar{\gamma}_{r n}^{2}-1}} \frac{\left\langle\psi_{n}\right\rangle_{a}^{2}}{\left\langle\psi_{n}^{2}\right\rangle_{v}}
$$

Since domain B is a straight tube, the specific acoustic impedance at $\mathcal{S}_{b}$ can be easily obtained in terms of $Z_{a}$ as

$$
Z_{b}=\rho_{b} c_{b} \frac{i Z_{a}+\rho_{b} c_{b} \tan \left(k t_{w}\right)}{i \rho_{b} c_{b}+Z_{a} \tan \left(k t_{w}\right)}
$$


For domain $\mathrm{C}$, since the boundaries in both the $x$ and $r$ directions are incomplete, the wavenumbers in these directions cannot be determined. Therefore, the wave expansion method applied previously is not available. Here, we use Green's function method to calculate the acoustic impedance at $\mathcal{S}_{c}$. The pressure inside domain $\mathrm{C}$ is a combination of the radiation fields at its continuous boundaries $\mathcal{S}_{b}$ and $\mathcal{S}_{c}$ and reads

$$
p_{c} \approx i k \rho c\left\{\bar{v}_{b} \int_{\mathcal{S}_{b}} G_{c}\left(x, r \mid x^{\prime}, r^{\prime}\right) d \mathcal{S}+\bar{v}_{c} \int_{\mathcal{S}_{c}} G_{c}\left(x, r \mid x^{\prime}, r^{\prime}\right) d \mathcal{S}\right\}
$$

The acoustic impedance at boundaries $\mathcal{S}_{b}$ and $\mathcal{S}_{c}$ are then easily presented as

$$
Z_{b}=\frac{i k_{c} \rho_{c} c_{c}}{\mathcal{S}_{b}}\left\{\mathcal{S}_{b}^{2} G_{b b}+\frac{\bar{v}_{c}}{\bar{v}_{b}} \mathcal{S}_{b} \mathcal{S}_{c} G_{b c}\right\}
$$

and

$$
Z_{c}=\frac{i k \rho c}{\delta_{c}}\left\{\delta_{c}^{2} G_{c c}+\frac{\bar{v}_{b}}{\bar{v}_{c}} \delta_{b} \delta_{c} G_{b c}\right\}
$$

respectively. Exploiting these two expressions leads to the explicit form of the acoustic impedance $Z_{c}$ as below

$$
Z_{c}=i \delta_{c} k_{c} \rho_{c} c_{c}\left\{G_{c c}+\frac{G_{b c}^{2}}{\frac{Z_{b}}{i \delta_{b} k_{c} \rho_{c} c_{c}}-G_{b b}}\right\}
$$

Where $G_{c}\left(x, r \mid x^{\prime}, r^{\prime}\right)$, is Green's function of "closed" domain C and reads [59]

$$
G_{c}\left(r, x \mid r^{\prime}, x\right)=\frac{1}{k_{c}^{2} V_{c}} \sum_{n, m=0}^{\infty} \frac{\vartheta_{n m}(r, x) \vartheta_{n m}\left(r^{\prime}, x^{\prime}\right)}{\left(\bar{\xi}_{n m}^{2}-1\right)\left\langle\vartheta_{n m}^{2}\right\rangle_{C}}
$$

Here, $V_{c}=h_{s} \mathcal{S}_{b}$ is the volume of the domain C. $\bar{\xi}_{n m}=\xi_{n m} / k_{c}$, where $\xi_{n m}^{2}=\xi_{r n}^{2}+\xi_{x m}^{2}$, denotes the normalized wavenumber of the closed domain C. $\xi_{r n}$ and $\xi_{x m}=m \pi / h_{s}$ 
represent the wavenumbers in the $r$ and $x$ directions, respectively. $\vartheta_{n m}(r, x)=$ $\phi_{r n}(r) \phi_{x m}(x)$ is the wavefunction of the "closed" domain C, the wavefunctions of the $r-$ and $x$-directional components read, respectively,

$$
\begin{aligned}
& \phi_{r n}(r)=J_{0}\left(\xi_{r n} r\right)-\alpha_{c n} Y_{0}\left(\xi_{r n} r\right) \text { and } \\
& \phi_{x m}(x)=\cos \left(\xi_{x m}\left(z-L_{a}-t_{w}\right)\right)
\end{aligned}
$$

The radial wavenumber $\xi_{r n}$ can be determined by applying the sound hard boundary conditions similarly as one stated in equation (5.3). Employing equation (5.12) and equation (5.13), the explicit form of the averaged Green's functions can be found as

$$
\begin{aligned}
& \left\langle G_{c}\right\rangle_{b b}=\frac{-1}{k_{c}^{2} V_{c}}\left\{1-\sum_{n=1}^{\infty} \frac{2}{\bar{\xi}_{x n}^{2}-1}\right\} \\
& \left\langle G_{c}\right\rangle_{c c}=\frac{-1}{k_{c}^{2} V_{c}}\left\{1-\sum_{n=1}^{\infty} \frac{\phi_{r n}^{2}\left(R_{c}\right)}{\left(\bar{\xi}_{r n}^{2}-1\right)\left\langle\phi_{r n}^{2}\right\rangle_{b}}\right\} \\
& \left\langle G_{c}\right\rangle_{b c}=\frac{-1}{k_{c}^{2} V_{c}}
\end{aligned}
$$

Finally, the acoustic impedance at $\delta_{d}$ will be derived. In the slit neck D, the pressure and velocity fields take the forms of

$$
\begin{aligned}
& p_{d}=D \varphi_{0}(r)=D\left[J_{0}(k r)-\alpha_{d} Y_{0}(k r)\right] \text { and } \\
& v_{d}=\frac{i}{k \rho_{d} c_{d}} \frac{\partial p_{d}}{\partial r}=-\frac{i}{\rho_{d} c_{c}} D\left[J_{1}(k r)-\alpha_{d} Y_{1}(k r)\right]
\end{aligned}
$$

Presenting the acoustic impedance at $\delta_{c}$ as $Z_{c}=p_{d}\left(R_{c}\right) / v_{d}\left(R_{c}\right)$, the ratio $\alpha_{d}$ in the wavefunction in domain $\mathrm{D}$ can be determined as 


$$
\alpha_{d}=\frac{\rho_{d} c_{d} J_{0}\left(k R_{c}\right)+i Z_{c} J_{1}\left(k R_{c}\right)}{\rho_{d} c_{d} Y_{0}\left(k R_{c}\right)+i Z_{c} Y_{1}\left(k R_{c}\right)}
$$

The acoustic impedance at the inlet of the SHR, eventually, can be found as

$$
Z_{h}=Z_{d}=\left.\frac{p_{d}}{v_{d}}\right|_{r=R}=i \rho_{d} c_{d} \frac{J_{0}(k R)-\alpha_{d} Y_{0}(k R)}{J_{1}(k R)-\alpha_{d} Y_{1}(k R)}
$$

When the thermal viscosity is considered, the released energy through heat mainly occurs inside the neck at resonant frequencies, since the velocity inside the SHR reaches maximum due to the abrupt reduction of the cross-sectional area at the ends of the neck. For the slit-type neck, effective mass density $\left(\rho_{e}\right)$ and sound velocity $\left(c_{e}\right)$ are considered [99], taking into account the thermal viscous effect,

$$
\rho_{e}=\frac{\rho}{\phi_{v}} \text { and } c_{e}=c \sqrt{\frac{\phi_{v}}{\gamma-(\gamma-1) \phi_{h}}},
$$

where $\rho, c$ and $\gamma$ are the mass density, sound velocity, and ratio of specific heat of air, respectively. $\phi_{v}$ and $\phi_{h}$ are viscosity-geometry and thermal-geometry functions for slits [see equation (4.7)]. Being side-branched to a duct and under an external excitation, the air layer at the inlet oscillates and radiates energy back into the upstream and downstream of the duct symmetrically. The SHR, therefore, performs as a monopole and the radiation pressure field can be formulated through Green's function the following.

$$
p_{s}(r, x)=-i k \rho c v_{d} \mathcal{S}_{d} \frac{1}{\mathcal{S}_{d}} \int_{\mathcal{S}_{d}} G\left(r, x \mid R, x^{\prime}\right) d \mathcal{S}
$$


Where, $G\left(r, x \mid r, x^{\prime}\right)$ is the Green's function of the duct,

$$
G\left(r, x \mid r, x^{\prime}\right)=\sum_{n=0}^{\infty} \frac{\psi_{n}(r) \psi_{n}\left(r^{\prime}\right)}{2 i k_{n} \mathcal{S}_{0}\left\langle\psi_{n}^{2}\right\rangle_{\mathcal{S}_{0}}} e^{-i k_{n}\left|x-x^{\prime}\right|}
$$

Here, $\psi_{n}(r)=J_{0}\left(\gamma_{n} r\right) ; \gamma_{n}$ is the $\mathrm{n}$-th order radial eigenvector of the duct's cross-section; and $k^{2}=k_{0}^{2}=k_{n}^{2}+\gamma_{n}^{2}$. Then the total pressure field inside the duct is

$$
p=p_{i} e^{-i k x}+p_{s}(r, x)
$$

Evaluating the pressure at the inlet of the SHR give the specific input acoustic impedance of the SHR,

$$
Z_{\text {in }}=\frac{1}{v_{d}}\left\{\frac{1}{\mathcal{S}_{d}} \int_{\mathcal{S}_{d}} p_{i} e^{-i k x} d \mathcal{S}_{d}\right\}=Z_{h}+\sigma \rho c F(k, h, R)=Z_{h}+Z_{c r}
$$

Where, space opening ratio of the $\mathrm{SHR}, \sigma=\mathcal{S}_{d} / \mathcal{S}_{0}$, the specific impedance of the radiation field, $Z_{c r}=\sigma \rho c F(k, h, R)$ and

$$
F(k, h, R)=\sum_{n=0}^{\infty} \frac{k}{k_{n}} \frac{1-i k_{n} h-e^{-i k_{n} h}}{\left(k_{n} h\right)^{2}}
$$

Acoustically, the radiation field comprises far-field and near-field components. The farfield component is induced by the fundamental mode and conserved while propagating outward, whereas the near-field component is produced by the higher-order modes of the duct and decays exponentially away from the inlet. Thus, the far-field component represents losing energy and its corresponding specific impedance is purely resistive [105]. The energy carried by the near-field component, in contrast, is preserved and oscillates in the vicinity of the inlet. Its corresponding impedance is purely reactive $[110,114]$. Aside from the impedance at the inlet, the input impedance of the SHR as expressed on equation 
(5.22), therefore, needs to be taken into consideration for the radiation impedance, denoted as

$$
Z_{\text {in }}=Z_{h}+\mathcal{R}-i \chi
$$

in which $\mathcal{R}$ and $\chi$ are the resistance and mass reactance corresponding to the far-field radiation and the near-field component, respectively. For traditional Helmholtz resonators, $\mathcal{R}=\sigma \rho c / 2$ and $\chi$ can be approximately estimated through neck length correction [47]. Here, they can be estimated from equation (5.23). $Z_{c r}=Z_{h}-i \chi$ is the corrected specific acoustic impedance of the SHR [114]. The resonances of the SHR are then determined under the condition of zero reactance of the corrected specific impedance $\left(\operatorname{Img}\left\{Z_{h}\right\}=\chi\right)$. It is noteworthy that for the non-viscous SHR, Zh is purely imaginary (reactive). The resonant frequencies of the side-branched SHR are lower than those of an isolated SHR whose resonances are determined from the condition $\operatorname{Img}\left\{Z_{h}\right\}=0$ because $\chi<0$ and $\operatorname{Img}\left\{Z_{h}\right\}$ are characterized by a positive-slope curve. Evaluating the pressure at the farfield, $|x| \rightarrow \infty$ gives the transmission and reflection coefficients

$$
T_{h}=1-\alpha^{2} \frac{\sigma \rho c}{Z_{h}+Z_{c r}} \text { and } R_{h}=-\alpha^{2} \frac{\sigma \rho c \vartheta^{2}}{Z_{h}+Z_{c r}}
$$

Here, $\alpha=\sin (k h / 2) /(k h / 2), \vartheta=e^{-i k x_{0}}$ and $x_{0}$ is the coordinate of the center of $\mathcal{S}_{d}$. When $k h \ll 1$, the first term of $F(k, h, R)$ approximately equals $1 / 2$ and the simplifying equation (5.25) leads to 


$$
T_{h}=1-\frac{\mathcal{R}}{\mathcal{R}+Z_{c r}} \text { and } R_{h}=-\frac{\mathcal{R} \vartheta^{2}}{\mathcal{R}+Z_{c r}},
$$

respectively. One can clearly see $T_{h}-R_{h}=1$, which completely differs from the case of the thin membrane [57, 58], i.e., $T_{h}+R_{h}=1$. This is due to the fact that the continuous air pressure field inside the duct leads to the in-phase $T_{h}$ and $R_{h}$, while in the thin membrane scenario, $T_{h}$ and $R_{h}$ are out-of-phase. For a lossless side-branched SHR mounted on the duct, its radiating pressure fields at the resonances in the downstream possess the same amplitudes as the incident pressure field, but are out of phase, leading to TL peaks (TL $=$ $\left.-20 \log _{10}\left|T_{h}\right|\right)$. This observation is readily confirmed by equation (5.26), showing that $T_{h}=0$ when $Z_{c r}=0$. In general, such a SHR possesses multiple resonances. The first resonance, which happens to be the strongest with the lowest frequency, gives the largest TL along with the highest Q-factor. In this dissertation, we focus only on the first SHR resonance.
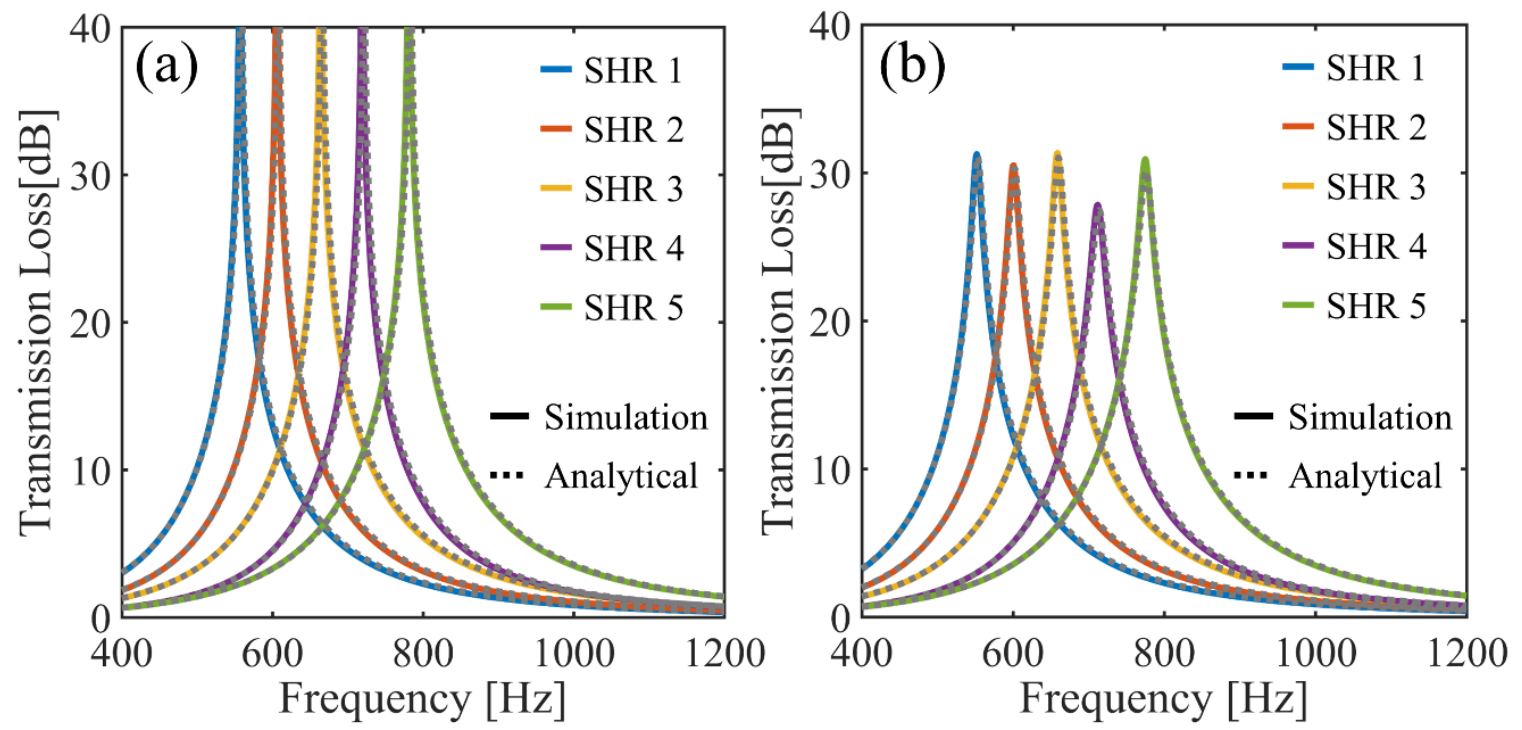

Figure 5.2. (a) and (b) TL spectra of non-viscous and viscous SHRs, respectively. The dashed curves correspond to analytical results, whereas the solid ones represent the simulation results. 


\subsection{Design acoustic silencer}

(a)

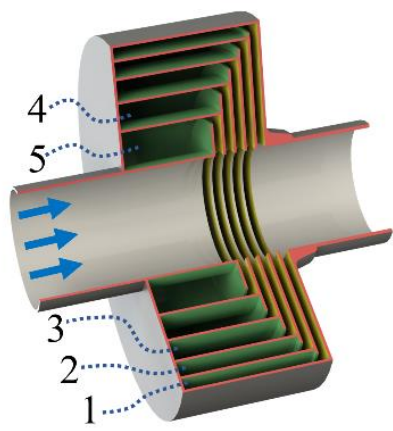

(b)
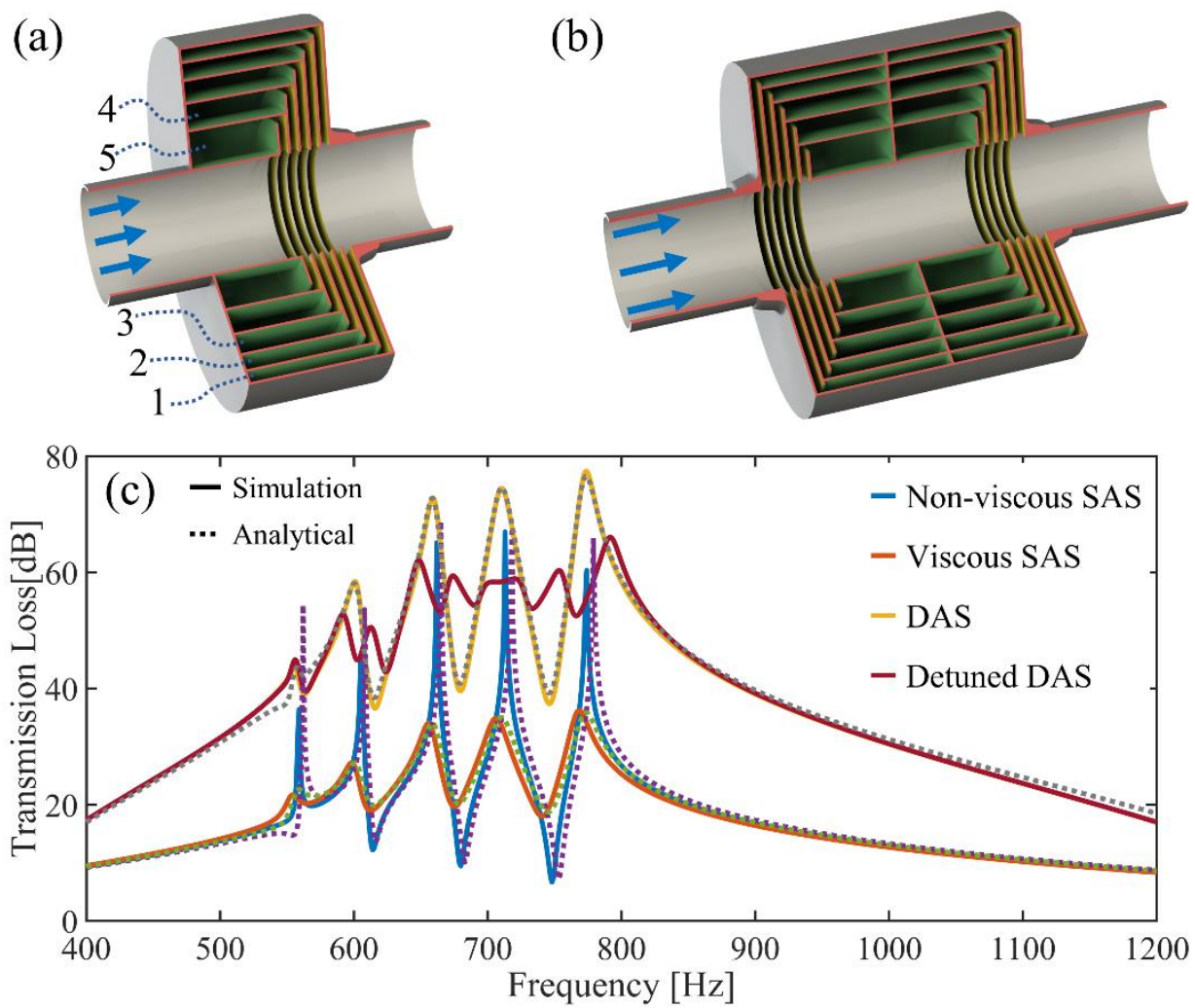

Figure 5.3. (a) and (b) Sectional views of a single-layer acoustic silencer consisting of five labeled SHRs and the double-layer acoustic silencer containing two single-layer acoustic silencers. (c) TL spectra of the non-viscous (NVC) and viscous (VC) single-layer acoustic silencers from analytical and numerical calculations. (d) Comparison between the viscous single-layer and double-layer silencers and the detuned viscous double-layer silencer. Here, S and A correspond to simulation and analytical results, respectively.

Five SHRs with different geometrical parameters are assembled compactly to form a subwavelength single-layer acoustic silencer (SAS), as illustrated in Figure 5.3(a). They are designed such that their working frequencies are distributed evenly within the working band of $0.5-1 \mathrm{kHz}$. Conducting a parametric study using the numerical software COMSOL Multiphysics allows us to determine the optimal geometrical parameters of each SHR, see Table 5.2. To simplify the numerical model, the thicknesses of all SHRs are set as $t_{w}=1$ $\mathrm{mm}$, which is feasible for 3D printing. The TL spectra of the non-viscous and viscous SHRs are calculated numerically and analytically, as presented in Figure 5.2(a) and Figure 5.2(b), respectively. Great agreement can be visualized between the theoretical prediction and the 
simulations, indicating that the developed theoretical model of the single SHR is reliable for the design and optimization purposes.

Table 5.2. The dimensions of the individual SHRs.

\begin{tabular}{cccccc}
\hline & First SHR & Second SHR & Third SHR & Fourth SHR & Fifth SHR \\
\hline$t_{w}(\mathrm{~mm})$ & 1.0 & 1.0 & 1.0 & 1.0 & 1.0 \\
$R_{a}(\mathrm{~mm})$ & 65.5 & 58.0 & 49.0 & 39.5 & 23.5 \\
$h_{a}(\mathrm{~mm})$ & 6.0 & 6.5 & 8.0 & 8.5 & 15.0 \\
$L_{a}(\mathrm{~mm})$ & 58.5 & 52.5 & 48.5 & 44.5 & 40.5 \\
$h(\mathrm{~mm})$ & 4.3 & 4.0 & 4.0 & 3.0 & 3.0 \\
\hline
\end{tabular}

Since the necks of the SHRs within a SAS are subwavelength and compactly stacked (24.3 $\mathrm{mm}$, less than $\lambda / 14$ at $1 \mathrm{kHz}$ ), there may exist a near-field interaction between adjacent SHRs. The SAS, therefore, cannot be treated as a combination of isolated SHRs connected in parallel. To take into account the interaction, Green's function method is again employed. Consider a model comprising of N slit-type Helmholtz resonators side branched into a duct. The coordinate of the SHRs' inlets are $x_{1}, x_{2}, . ., x_{N}$. The pre-corrected specific acoustic impedance of the SHRs are $Z_{h 1}, Z_{h 2}, . ., Z_{h n}$. Employing the Green's function method as previously gives the radiation field from the SHRs' inlets back to the duct as

$$
p_{s}(r, x)=-i k \rho c \sum_{m=1}^{N} \bar{v}_{m} \int_{\mathcal{S}_{m}} G\left(r, x \mid R, x^{\prime}\right) d \mathcal{S}_{m}
$$

Evaluating equation (5.27) at the inlet of the $n$th neck gives

$$
\bar{p}_{n}=\lambda_{n} \alpha_{n} P_{i}-\rho c \sum_{m=1}^{N} \sigma_{m} \bar{v}_{m} A_{n m}\left(k, h_{n}, h_{m}, R\right)
$$


Where,

$$
A_{n m}=\frac{i k \mathcal{S}_{0}}{\mathcal{S}_{n} \mathcal{S}_{m}} \int_{\mathcal{S}_{n}} \int_{\mathcal{S}_{m}} G\left(R, z \mid R, z^{\prime}\right) d \mathcal{S}_{m} d \mathcal{S}_{n}
$$

Plugging equation (5.19) into equation (5.29) leads to

$$
\begin{aligned}
A_{n m}=\delta_{n m} \sum_{s=0}^{\infty} \frac{k}{k_{s}} \frac{1-i k_{s} h_{n}-e^{-i k_{s} h_{n}}}{\left(k_{s} h_{n}\right)^{2}} \\
\quad+\left(1-\delta_{n m}\right) \frac{1}{2} \sum_{s=0}^{\infty} \frac{k}{k_{s}} e^{-i k_{s}\left|x_{n}-x_{m}\right|} \frac{\sin \left(k_{s} h_{n} / 2\right)}{k_{s} h_{n} / 2} \frac{\sin \left(k_{s} h_{m} / 2\right)}{k_{s} h_{m} / 2}
\end{aligned}
$$

Employing the definition, $Z_{h n}=\bar{p}_{n} / \bar{v}_{n}$, results

$$
\lambda_{n} \alpha_{n} v_{i}=\sum_{m=1}^{N}\left(\delta_{n m} \bar{Z}_{h n}+A_{n m} \sigma_{m}\right) \bar{v}_{m}=\sum_{m=1}^{N} \mathcal{F}_{n m} \bar{v}_{m}
$$

Here, $\mathcal{F}_{n m}=\delta_{n m} \bar{Z}_{h n}+A_{n m} \sigma_{m}$ and $\bar{Z}_{h n}=Z_{h n} / \rho c$. Plugging the SHRs' inlet velocity solved from equation (5.31) into the radiation field evaluated at far-field gives the reflection and transmission fields

$$
p_{r}(x)=-\frac{P_{i} e^{i k x}}{2} \sum_{n=1}^{N} \sum_{m=1}^{N}\left(\sigma_{n} \alpha_{n} v_{n}\right)\left[\mathcal{F}^{-1}\right]_{n m}\left(\alpha_{m} v_{m}\right)
$$

and

$$
p_{t}(x)=\left(1-\frac{1}{2} \sum_{n=1}^{N} \sum_{m=1}^{N}\left(\sigma_{n} \alpha_{n} / v_{n}\right)\left[\mathcal{F}^{-1}\right]_{n m}\left(\alpha_{m} v_{m}\right)\right) p_{i}
$$


The resultant transmission and reflection coefficients are readily obtained in the following matrix forms

$$
\begin{aligned}
& T=1-\frac{1}{2} \sum_{n=1}^{N} \sum_{m=1}^{N}\left(\sigma_{n} \alpha_{n} \vartheta_{n}^{-1}\right)\left[\boldsymbol{F}^{-1}\right]_{n m}\left(\alpha_{m} \vartheta_{m}\right), \\
& R=-\frac{1}{2} \sum_{n=1}^{N} \sum_{m=1}^{N}\left(\sigma_{n} \alpha_{n} \vartheta_{n}^{-1}\right)\left[\boldsymbol{F}^{-1}\right]_{n m}\left(\alpha_{m} \vartheta_{m}\right),
\end{aligned}
$$

Here, $N=5, \sigma_{n}=\mathcal{S}_{d n} / \mathcal{S}_{0}, \alpha_{n}=\sin \left(k h_{n} / 2\right) /\left(k h_{n} / 2\right)$, and $\vartheta_{n}=e^{-i k x_{n}}$, in which $\mathcal{S}_{d n}$, $h_{n}$, and $x_{n}$ denote the cross-sectional area, height, and coordinate of the nth SHR inlet, respectively. $\boldsymbol{F}$ is the coupling impedance matrix (see Ref. [65]). The calculated TL profiles of the non-viscous and the viscous SASs, plotted in Figure 5.3(c), show great agreement between the developed analytical solution [equation (5.34) and equation (5.35)] and simulation results. For the non-viscous SAS, the TL peaks slightly shift, compared to the TL peaks of individual SHRs, due to the weak interaction between the adjacent SHRs [65]. In addition, the TL outside the resonance region is significantly enhanced, which can be explained by the additive property of the coupling of adjacent slit necks in the non-resonant region. Within the resonant region, the dips lying between two adjacent TL peaks indicate TL even lower than those of the individual SHRs at the same frequency, owing to the Fano resonance induced by the coupling mainly between two adjacent SHR resonances [82, 83].

Since the existence of sharp dips causes a non-optimal TL profile, we introduce high viscosity into the SHRs to make the TL profile less fluctuating. Because of the abrupt change in the cross-sectional areas of the neck inlet and the neck outlet, the velocity fields inside are strong at the resonances, leading to large slipping velocity fields between the air 
layers inside the viscous boundary layer. The massive friction area of the neck, therefore, guarantees a huge amount of acoustic energy to be released through the heat induced from the friction force between the slipping air layers. As a result, the peaks or the dips caused by the resonances become less sharp. The effect of the introduced viscosity on the TL performance can be seen directly from Figure 5.2(b) for the SHRs and Figure 5.3(c) for the SAS. Far away from the resonances, where the slipping velocity fields are weak, the viscosity is negligible. Within the resonance region, the influence of the viscosity is maximized.

To further improve the sound insulation performance, two SASs are compactly assembled to form a double-layer acoustic silencer (DAS), as illustrated in Figure 5.3(b), which in theory doubles the overall TL of the SAS. This prediction is confirmed by Figure 5.3(d) for both the non-viscous and the viscous cases. (The TL deteriorates as the two SASs are brought closer due to the emergence of non-negligible SHR couplings, see Ref. [65]) To interpret this, we treat the two SASs as two acoustic impedance layers, separated by $L_{e}$, whose transmission and reflection coefficients are denoted by $\left(T_{1}\right.$ and $\left.T_{2}\right)$ and $\left(R_{1}\right.$ and $\left.R_{2}\right)$, respectively. In this way, the total transmission and the total reflection coefficients, respectively, take the forms

$$
\begin{aligned}
& T=\frac{\vartheta_{e} T_{1} T_{2}}{1-R_{1} R_{2} \vartheta_{e}^{2}}, \\
& R=R_{1}+\frac{T_{1}^{2} R_{2} \vartheta_{e}^{2}}{1-R_{1} R_{2} \vartheta_{e}^{2}},
\end{aligned}
$$

where $\vartheta_{e}=e^{-i k L_{e}}$. The effectiveness of equation (5.36) and equation (5.37) is validated in the Ref. [65]. The TL reaches its maxima when $\left|T_{1}\right| \rightarrow 0$ or $\left|T_{2}\right| \rightarrow 0$, corresponding to 
the SAS resonance frequencies. It is implied that the SAS and the DAS are expected to exhibit TL peaks at the same frequencies. Moreover, when identical SASs, namely, $T_{1}=$ $T_{2}$ and $R_{1}=R_{2}$, are chosen, the magnitude of the transmission reads $|T|=$ $\left|T_{1}\right|^{2} /\left|1-\left(\vartheta R_{1}\right)^{2}\right|$. The TL of the DAS is then approximated, in the vicinity of the resonances, as $2 \mathrm{TL}_{\mathrm{SAS}}+40 \log _{10} 4 \pi L / \lambda$ and around the antiresonances $(\mathrm{T} \approx 1)$ as $\mathrm{TL}_{\mathrm{DAS}} \approx 2 \mathrm{TL}_{\mathrm{SAS}}$, which clearly suggests that $\mathrm{TL}_{\mathrm{DAS}}$ is at least twice as high as TLSAS. Although the DAS dramatically improves the sound insulation performance, the occurrence of the fluctuating TL profile remains unsolved, even in the presence of strong viscosity. To address this issue, we slightly detune the resonance frequencies of the SHRs by shifting their separations by $3.5 \mathrm{~mm}$. Consequently, additional peaks and dips appear. Although the detuning suppresses the TL around the resonances, the TL performance is improved overall to be at least $40 \mathrm{~dB}$ over the frequency range of $0.54-0.88 \mathrm{kHz}$. As for the ventilation efficiency, since the proposed designs support the straight airflow path with a constant cross section, the ventilation remains perfect even in the presence of the DAS (see Ref. [65]). 


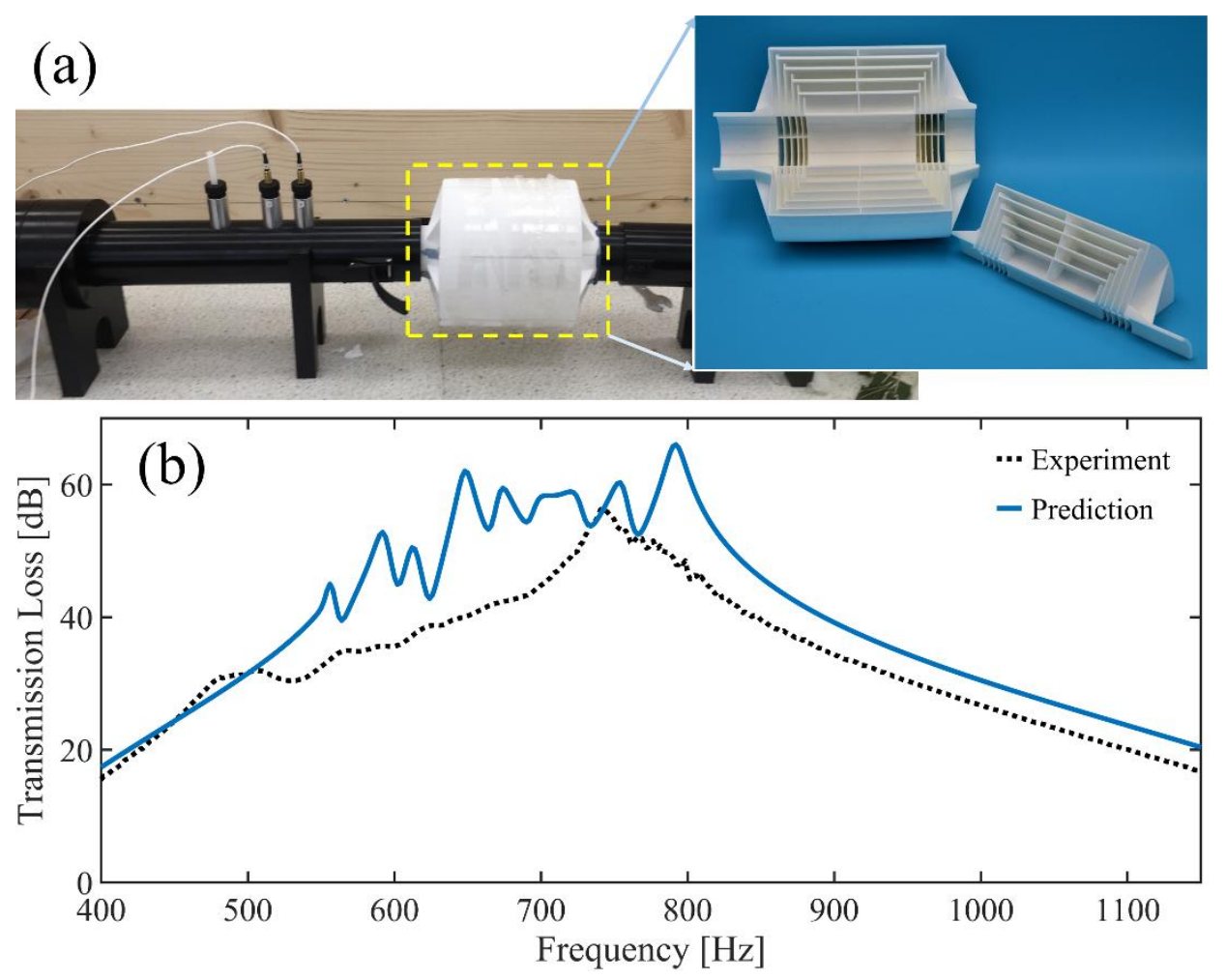

Figure 5.4. (a) Photograph of the experimental setup including a Mecanum Inc. standard impedance tube and a detuned double-layer acoustic silencer sample [see the inset]. (b) Comparison between the numerical

(solid curve) and the experimental results (dashed curve) of the detuned double-layer acoustic silencer design.

To experimentally examine the viscous, detuned DAS design, we build the test bed shown in Figure 5.4(a). The detuned DAS sample [see the inset of Figure 5.4(a)] is fabricated from nylon using a Formlabs 3D printer. To ease the printing difficulty, we divide the DAS sample into six pieces and print them separately. The final DAS sample is then assembled by gluing all six pieces. Since both the theoretical and the simulation models assume the acoustically rigid shells of the silencer, the DAS is reinforced by thin supporting walls at the middle plane of each piece. The inner and outer diameters and the total length of the fabricated DAS are $44.5 \mathrm{~mm}, 145 \mathrm{~mm}$, and $130 \mathrm{~mm}$, respectively. This silencer, thus, can be considered as an acoustically subwavelength device compared with the working wavelength of $490 \mathrm{~mm}$ at the working band center $(0.7 \mathrm{kHz})$. As shown in Figure 5.4(b), the experimental measurement is in good agreement with the corresponding numerical 
simulation. The TL is observed to be greater than $20 \mathrm{~dB}$ across a broadband covering $0.43-$ $1.1 \mathrm{kHz}$. More importantly, within the range of $0.48-1 \mathrm{kHz}$, the TL exceeds $30 \mathrm{~dB}$ with the maximum over $50 \mathrm{~dB}$, proving excellent sound insulation performance. The derivation between the experimental and the numerical results may originate from the imperfect assembling of DAS pieces, which leads to the acoustic leakage from the DAS to the tube and the weak acoustic interaction between adjacent DAS pieces. These imperfections weaken the pressure intensity inside the SHRs at their resonance frequencies, resulting in the smooth curve instead of the fluctuating profile shown in simulations. Furthermore, the working band could be broadened by adjusting the geometrical parameters of the SHRs or by packing more SHRs. It has been reported that the working band can become much broader if the first-order and the second-order resonances are brought close [93]. Due to the challenging control of both the fundamental and higher-order resonances in such a passive resonator, this work is, hence, focused only on exploring the first-order resonances of the SHRs.

\subsection{Conclusion}

Based on the slit-type Helmholtz resonators, we have designed and demonstrated a compact subwavelength acoustic silencer, named double-layer acoustic silencer, which realizes strong acoustic insulation applications over the broadband of $0.48-0.95 \mathrm{kHz}$ while preserving perfect ventilation. All the involved structures have been studied analytically using a complete theoretical model. The simulations and experiments of this double-layer acoustic silencer show excellent broadband sound insulation performance: the transmission loss, serving as the measure of the sound insulation performance, maintains at least $20 \mathrm{~dB}$ over the range of $0.43-1.1 \mathrm{kHz}$, even greater than $30 \mathrm{~dB}$ within the region of $0.48-0.95$ 
$\mathrm{kHz}$, and reaches the maximum exceeding $50 \mathrm{~dB}$. The overall transmission loss across the working band has been optimized by introducing additional viscosity and detuning the frequencies of the individual slit-type Helmholtz resonators. The proposed designs, supporting straight airflow path with a constant cross section, can maintain the excellent ventilation when deployed in a duct environment. Finally, our proposed subwavelength compact acoustic silencer paves the way toward the related future sound insulation devices. 


\section{Chapter 6. Conclusion}

In this dissertation, we have employed the idea of metamaterials on designing noise control devices working on noise isolation, noise energy harvesting, noise silencing, and noise barrier. First, by exploiting the negative mass density around the anti-resonance of MAM, which strongly suppresses transmitted noise, a lightweight and optical transparent acoustic panel was designed. Double MAM-layer structure was used to enhance significantly unit cell's STL performance and bending stiffness of panel, which leads to ease the negative effect of panel's frame vibration on the STL performance. Numerical simulations were conducted to optimize the structure and performance of the panel. Experimental results show excellent performance, i.e., STL exceeds $20 \mathrm{~dB}$ within the range $0.32-2.5 \mathrm{kHz}$ and ranges $40-59 \mathrm{~dB}$ within $0.45-1.48 \mathrm{kHz}$, which deeply breaks the mass density law.

In addition to applications on noise isolation, the first local resonance of MAM is employed to harvest acoustic energy highly efficiently. The harvester comprises of a MAM and a magnet coin fixed right behind a magnet coil attached to the mass of MAM. Once the first local resonance of MAM is excited, the maximum oscillation of the magnet coil in the static magnetic field is generated. The oscillation induces a current inside the magnet coil, and acoustic energy is converted to electric energy through a resistor connected to the magnet coil. Theoretical analysis was developed and validated with numerical simulation results. The optimal results show that the conversion and harvesting coefficient can reach $48 \%$ and $36 \%$ over the maximum of $50 \%$, which are truly remarkable results for acoustic harvesters. 
Second, slit-type Helmholtz resonators and double-layer structures are employed to design compact and broadband acoustic silencers with ventilation. Complete theoretical models of both individual resonators and layers of resonators are developed and well-validated with numerical simulations. The optimal result is validated with the experimental result as well. It says STL is more than $20 \mathrm{~dB}$ over the range $0.43-1.1 \mathrm{kHz}$ and $30 \mathrm{~dB}$ within the range $0.48-0.95 \mathrm{kHz}$ and exceeds $50 \mathrm{~dB}$ at its maximum.

Finally, an ultra-broadband acoustic barrier was designed. Wherein, resonances induced in space coiling channels couple with continuum background supported by ventilation channels, resulting in a coupling resonance called Fano resonance, which strongly blocks noise and airflow. Theoretical analysis of the unit cell was established to explain the forming of Fano resonances. Moreover, negative effective mass density and bulk modulus were realized at the Fano resonances as well. Acoustic hyper-damping was introduced by inserting thin acoustic foam into the coiling channel's velocity anti-nodes to optimize the barrier's STL performance curve. Experiments show remarkable results, i.e., STL is at least $10 \mathrm{~dB}$ within $0.44-3.85 \mathrm{kHz}$ with an average of $17 \mathrm{~dB}$, and average absorption is $65 \%$ over this ultra-broadband range, while the open ratio is still high, $29.25 \%$.

We believe our proposed designs will have tremendous application potential for noise control, and the developed theoretical models will lay a strong foundation for developing high efficient acoustic panels, silencers, and barriers with high ventilation and sound energy harvesting. Furthermore, there are many opening and promising directions related to our works we plan to conduct in the near future: (1) we will develop a homogenization model for magnetic-based acoustic metamaterials to find the coupling coefficients between vibroacoustic model and electric model mathematically. From that, the condition for 
perfect absorption, conversion, and harvesting can be determined theoretically, leading to super acoustic harvesters. (2) Since in our current silencer designs, there still have backpressure that in many cases give negative effects on noise source devices, resonator coupling for perfect absorption and optimization will be investigated to design both high transmission loss and absorption silencers. (3) Microperforated shells can be employed on our current acoustic barrier design to improve the overall absorption coefficient. We believe these planned studies will broaden potential applications of our research and bring it closer to industrial applications. 


\section{References}

1. Yang, Z., et al., Membrane-type acoustic metamaterial with negative dynamic mass. Phys Rev Lett, 2008. 101(20): p. 204301.

2. Yang, Z., et al., Acoustic metamaterial panels for sound attenuation in the 50-1000 Hz regime. Applied Physics Letters, 2010. 96(4).

3. Mei, J., et al., Dark acoustic metamaterials as super absorbers for lowfrequency sound. Nat Commun, 2012. 3: p. 756.

4. Ma, G., et al., Acoustic metasurface with hybrid resonances. Nat Mater, 2014. 13(9): p. 873-8.

5. Shelby, R.A., D.R. Smith, and S. Schultz, Experimental verification of a negative index of refraction. science, 2001. 292(5514): p. 77-79.

6. Shalaev, V.M., Optical negative-index metamaterials. Nature photonics, 2007. 1(1): p. 41-48.

7. Engheta, N. and R.W. Ziolkowski, A positive future for double-negative metamaterials. IEEE Transactions on Microwave Theory and Techniques, 2005. 53(4): p. 1535-1556.

8. Cheng, Y., et al., Ultra-sparse metasurface for high reflection of lowfrequency sound based on artificial Mie resonances. Nat Mater, 2015. 14(10): p. 1013-9.

9. Seo, S.-H. and Y.-H. Kim, Silencer design by using array resonators for low-frequency band noise reduction. The Journal of the Acoustical Society of America, 2005. 118(4): p. 2332-2338. 
10. Wang, X., et al., Synergetic coupling large-scale plate-type acoustic metamaterial panel for broadband sound insulation. Journal of Sound and Vibration, 2019. 459.

11. Ghaffarivardavagh, R., et al., Ultra-open acoustic metamaterial silencer based on Fano-like interference. Physical Review B, 2019. 99(2).

12. Zhang, H.-1., et al., Omnidirectional ventilated acoustic barrier. Applied Physics Letters, 2017. 111(20).

13. Zhu, X., et al., Deep-Subwavelength-Scale Directional Sensing Based on Highly Localized Dipolar Mie Resonances. Physical Review Applied, 2016. 5(5).

14. Chen, Y., et al., Membrane-type acoustic metamaterials: Theory, design, and application. Theory and Design of Acoustic Metamaterials, 2015: p. 53-103.

15. Li, J., et al., Acoustic metamaterials capable of both sound insulation and energy harvesting. Smart Materials and Structures, 2016. 25(4).

16. Yang, Z., et al., Acoustic metamaterial panels for sound attenuation in the 50-1000 Hz regime. Applied Physics Letters, 2010. 96(4): p. 041906.

17. Huang, T.Y., C. Shen, and Y. Jing, Membrane- and plate-type acoustic metamaterials. Journal of the Acoustical Society of America, 2016. 139(6): p. 3239-3249.

18. Ma, F.Y., M. Huang, and J.H. Wu, Acoustic metamaterials with synergetic coupling. Journal of Applied Physics, 2017. 122(21): p. 215102. 
19. Ma, F., M. Huang, and J.H. Wu, Ultrathin lightweight plate-type acoustic metamaterials with positive lumped coupling resonant. Journal of Applied Physics, 2017. 121(1): p. 015102.

20. Ma, F., et al., A purely flexible lightweight membrane-type acoustic metamaterial. Journal of Physics D: Applied Physics, 2015. 48(17): p. 175105.

21. Chen, Y.Y., et al., Analytical coupled vibroacoustic modeling of membrane-type acoustic metamaterials: Membrane model. Journal of the Acoustical Society of America, 2014. 136(3): p. 969-979.

22. Chen, Y.Y., et al., Analytical coupled vibroacoustic modeling of membrane-type acoustic metamaterials: Plate model. Journal of the Acoustical Society of America, 2014. 136(6): p. 2926-2934.

23. Sui, N., et al., A lightweight yet sound-proof honeycomb acoustic metamaterial. Applied Physics Letters, 2015. 106(17): p. 171905.

24. Chen, X., et al., Active acoustic metamaterials with tunable effective mass density by gradient magnetic fields. Applied Physics Letters, 2014. 105(7): p. 071913.

25. Xiao, S., et al., Active control of membrane-type acoustic metamaterial by electric field. Applied Physics Letters, 2015. 106(9): p. 091904.

26. Airoldi, L. and M. Ruzzene, Design of tunable acoustic metamaterials through periodic arrays of resonant shunted piezos. New Journal of Physics, 2011. 13(11): p. 113010. 
27. Chen, S.-B., et al., Band gap control of phononic beam with negative capacitance piezoelectric shunt. Chinese Physics B, 2011. 20(1): p. 014301.

28. Gonella, S., A.C. To, and W.K. Liu, Interplay between phononic bandgaps and piezoelectric microstructures for energy harvesting. Journal of the Mechanics and Physics of Solids, 2009. 57(3): p. 621-633.

29. Bergamini, A., et al., Phononic Crystal with Adaptive Connectivity. Advanced Materials, 2014. 26(9): p. 1343-1347.

30. Li, J., et al., Acoustic metamaterials capable of both sound insulation and energy harvesting. Smart Materials and Structures, 2016. 25(4): p. 045013.

31. Akl, W. and A. Baz, Active Acoustic Metamaterial With Simultaneously Programmable Density and Bulk Modulus. Journal of Vibration and Acoustics-Transactions of the Asme, 2013. 135(3): p. 031001.

32. Roundy, S. and P.K. Wright, A piezoelectric vibration based generator for wireless electronics. Smart Materials \& Structures, 2004. 13(5): p. 1131-1142.

33. Aridogan, U., I. Basdogan, and A. Erturk, Random vibration energy harvesting on thin plates using multiple piezopatches. Journal of Intelligent Material Systems and Structures, 2016. 27(20): p. 2744-2756.

34. Tol, S., F.L. Degertekin, and A. Erturk, Phononic crystal Luneburg lens for omnidirectional elastic wave focusing and energy harvesting. Applied Physics Letters, 2017. 111(1). 
35. Xiao, S., S.T. Tang, and Z. Yang, Voltage-tunable acoustic metasheet with highly asymmetric surfaces. Applied Physics Letters, 2017. 111(19): p. 194101.

36. Lu, Z.B., M. Shrestha, and G.K. Lau, Electrically tunable and broaderband sound absorption by using micro-perforated dielectric elastomer actuator. Applied Physics Letters, 2017. 110(18): p. 182901.

37. Zhang, Q. and E.S. Kim, Vibration Energy Harvesting Based on Magnet and Coil Arrays for Watt-Level Handheld Power Source. Proceedings of the Ieee, 2014. 102(11): p. 1747-1761.

38. Mikoshiba, K., J.M. Manimala, and C.T. Sun, Energy harvesting using an array of multifunctional resonators. Journal of Intelligent Material Systems and Structures, 2013. 24(2): p. 168-179.

39. Ma, G.C., et al., Acoustic metasurface with hybrid resonances. Nature Materials, 2014. 13(9): p. 873-878.

40. Griffiths, D.J., Introduction to electrodynamics. Fourth edition. ed. 2013, Boston: Pearson. xviii, 599 pages.

41. Leissa, A.W., Vibration of plates. Nasa Sp-160. 1969, Washington: Scientific and Technical Information Division, NASA. vii, 353 pages.

42. Graff, K.F., Wave motion in elastic solids. 1991, New York: Dover Publications. xv, 649 pages.

43. Ingard, U., Transmission of sound through a stretched membrane. Journal of the Acoustical Society of America, 1954. 26(1): p. 99-101. 
44. Makris, S.E., C.L. Dym, and J.M. Smith, Transmission loss optimization in acoustic sandwich panels. The Journal of the Acoustical Society of America, 1986. 79(6): p. 1833-1843.

45. Antonio, J., A. Tadeu, and L. Godinho, Analytical evaluation of the acoustic insulation provided by double infinite walls. Journal of sound and vibration, 2003. 263(1): p. 113-129.

46. Bolton, J., N.-M. Shiau, and Y. Kang, Sound transmission through multipanel structures lined with elastic porous materials. Journal of sound and vibration, 1996. 191(3): p. 317-347.

47. Kinsler, L.E., et al., Fundamentals of Acoustics. 4 ed. 2000, New York: John Wiley \& Sons, Inc.

48. Bruneau, M., Fundamentals of acoustics. 2013: John Wiley \& Sons.

49. Cox, T.J. and P. D'antonio, Acoustic absorbers and diffusers: theory, design and application. 2009: Crc Press.

50. Cowan, J.P., Handbook of environmental acoustics. 1993: John Wiley \& Sons.

51. Harris, C.M., Handbook of acoustical measurements and noise control. 1991: McGraw-Hill New York.

52. Yao, S., X. Zhou, and G. Hu, Investigation of the negative-mass behaviors occurring below a cut-off frequency. New Journal of Physics, 2010. 12(10): p. 103025.

53. Cummer, S.A., J. Christensen, and A. Alù, Controlling sound with acoustic metamaterials. Nature Reviews Materials, 2016. 1(3). 
54. Ge, H., et al., Breaking the barriers: advances in acoustic functional materials. National Science Review, 2018. 5(2): p. 159-182.

55. Fang, N., et al., Ultrasonic metamaterials with negative modulus. Nature materials, 2006. 5(6): p. 452-456.

56. Yang, M., et al., Coupled membranes with doubly negative mass density and bulk modulus. Phys Rev Lett, 2013. 110(13): p. 134301.

57. Chen, Y., et al., Analytical coupled vibroacoustic modeling of membrane-type acoustic metamaterials: membrane model. J Acoust Soc Am, 2014. 136(3): p. 969.

58. Nguyen, H., et al., Analytical coupled modeling of a magneto-based acoustic metamaterial harvester. Smart Materials and Structures, 2018. 27(5).

59. Nguyen, H. Acoustic Metasurface Harvester. in INTER-NOISE and NOISE-CON Congress and Conference Proceedings. 2018. Institute of Noise Control Engineering.

60. Varanasi, S., et al., The low frequency performance of metamaterial barriers based on cellular structures. Applied Acoustics, 2013. 74(4): p. 485-495.

61. Naify, C.J., et al., Scaling of membrane-type locally resonant acoustic metamaterial arrays. J Acoust Soc Am, 2012. 132(4): p. 2784-92.

62. Varanasi, S., J.S. Bolton, and T. Siegmund, Experiments on the low frequency barrier characteristics of cellular metamaterial panels in a diffuse sound field. J Acoust Soc Am, 2017. 141(1): p. 602. 
63. Ang, L.Y.L., Y.K. Koh, and H.P. Lee, Plate-type acoustic metamaterials: Evaluation of a large-scale design adopting modularity for customizable acoustical performance. Applied Acoustics, 2019. 149: p. $156-170$.

64. Leissa, A.W. and M.S. Qatu, Vibrations of Continuous Systems. 2011, New York: McGraw-Hill.

65. Nguyen, H., et al., Broadband acoustic silencer with ventilation based on slit-type Helmholtz resonators. Applied Physics Letters, 2020. 117(13): p. 134103.

66. Nguyen, H., et al., A broadband acoustic panel based on double-layer membrane-type metamaterials. Applied Physics Letters, 2021. 118(18).

67. Pai, P.F. and G. Huang, Theory and design of acoustic metamaterials. 2015: SPIE Press.

68. Nguyen, H. and G. Huang. Perfect Absorption in Duct by Coupling Helmholtz Resonators. in INTER-NOISE and NOISE-CON Congress and Conference Proceedings. 2020. Institute of Noise Control Engineering.

69. Leissa, A.W., Vibration of Plates. 1969, Washington, D. C.: NASA.

70. Thota, M. and K.W. Wang, Reconfigurable origami sonic barriers with tunable bandgaps for traffic noise mitigation. Journal of Applied Physics, 2017. 122(15).

71. García-Chocano, V.M., S. Cabrera, and J. Sánchez-Dehesa, Broadband sound absorption by lattices of microperforated cylindrical shells. Applied Physics Letters, 2012. 101(18). 
72. Can, A., et al., Traffic noise spectrum analysis: Dynamic modeling vs. experimental observations. Applied Acoustics, 2010. 71(8): p. 764-770.

73. Ingard, U., Noise reduction analysis. 2009: Jones \& Bartlett Publishers.

74. Nguyen, H. and G. Huang. Multiple Helmholtz resonator based acoustic liner (Conference Presentation). in Health Monitoring of Structural and Biological Systems XIII. 2019. International Society for Optics and Photonics.

75. Hirsekorn, M., Small-size sonic crystals with strong attenuation bands in the audible frequency range. Applied Physics Letters, 2004. 84(17): p. 3364-3366.

76. Sanchez-Dehesa, J., et al., Noise control by sonic crystal barriers made of recycled materials. J Acoust Soc Am, 2011. 129(3): p. 1173-83.

77. Garc1'a-Chocano, V.M. and J. Sánchez-Dehesa, Optimum control of broadband noise by arrays of cylindrical units made of a recycled material. Applied Acoustics, 2013. 74(1): p. 58-62.

78. Koussa, F., et al. Transport noise reduction by low height sonic crystal noise barriers. 2012.

79. Liang, Z. and J. Li, Extreme acoustic metamaterial by coiling up space. Physical review letters, 2012. 108(11): p. 114301.

80. Ghaffarivardavagh, R., et al., Horn-like space-coiling metamaterials toward simultaneous phase and amplitude modulation. Nat Commun, 2018. 9(1): p. 1349.

81. Fano, U., Effects of Configuration Interaction on Intensities and Phase Shifts. Physical Review, 1961. 124(6): p. 1866-1878. 
82. Joe, Y.S., A.M. Satanin, and C.S. Kim, Classical analogy of Fano resonances. Physica Scripta, 2006. 74(2): p. 259-266.

83. Miroshnichenko, A.E., S. Flach, and Y.S. Kivshar, Fano resonances in nanoscale structures. Reviews of Modern Physics, 2010. 82(3): p. 22572298.

84. Rau, A., Perspectives on the Fano resonance formula. Physica Scripta, 2004. 69(1): p. C10.

85. Lee, T., et al., Fano-Like Acoustic Resonance for Subwavelength Directional Sensing: 0-360 Degree Measurement. Adv Sci (Weinh), 2020. 7(6): p. 1903101.

86. Ingard, U., On the Theory and Design of Acoustic Resonators. The Journal of the Acoustical Society of America, 1953. 25(6): p. 1037-1061.

87. Zhang, X. and Z. Liu, Negative refraction of acoustic waves in twodimensional phononic crystals. Applied Physics Letters, 2004. 85(2): p. 341-343.

88. Xie, Y., et al., Wavefront modulation and subwavelength diffractive acoustics with an acoustic metasurface. Nature communications, 2014. 5(1): p. 1-5.

89. Li, J., et al., A sound absorbing metasurface with coupled resonators. Applied Physics Letters, 2016. 109(9).

90. Zhou, Y., et al., Acoustic Multiband Double Negativity from Coupled Single-Negative Resonators. Physical Review Applied, 2018. 10(4).

91. Sun, M., et al., Broadband acoustic ventilation barriers. Physical Review Applied, 2020. 13(4): p. 044028. 
92. Xiong, L., W. Bi, and Y. Auregan, Fano resonance scatterings in waveguides with impedance boundary conditions. J Acoust Soc Am, 2016. 139(2): p. 764-72.

93. Yang, M., et al., Optimal sound-absorbing structures. Materials Horizons, 2017. 4(4): p. 673-680.

94. Liu, C., et al., Reversed Doppler effect based on hybridized acoustic Mie resonances. Scientific reports, 2020. 10(1): p. 1-7.

95. INGARD, U., Transmission of Sound through a Stretched Membrane. The Journal of the Acoustical Society of America, 1954. 26(99): p. 99101.

96. Nguyen, H.Q., et al., A Fano-based acoustic metamaterial for ultrabroadband sound barriers. Proceedings of the Royal Society A: Mathematical, Physical and Engineering Sciences, 2021. 477(2248).

97. Nguyen, H., et al., Channel-Coupling Fano Resonance and Acoustic Metadamping. arXiv preprint arXiv:2009.11014, 2020.

98. Allard, J. and N. Atalla, Propagation of sound in porous media: modelling sound absorbing materials 2e. 2009: John Wiley \& Sons.

99. Stinson, M.R., The propagation of plane sound waves in narrow and wide circular tubes, and generalization to uniform tubes of arbitrary cross-sectional shape. The Journal of the Acoustical Society of America, 1991. 89(2): p. 550-558.

100. Biot, M.A., Theory of Propagation of Elastic Waves in a Fluid-Saturated Porous Solid. I. Low-Frequency Range. The Journal of the Acoustical Society of America, 1956. 28(2): p. 168-178. 
101. Biot, M.A., Theory of Propagation of Elastic Waves in a Fluid-Saturated Porous Solid. II. Higher Frequency Range. The Journal of the Acoustical Society of America, 1956. 28(2): p. 179-191.

102. Johnson, D.L., J. Koplik, and R. Dashen, Theory of dynamic permeability and tortuosity in fluid-saturated porous media. Journal of fluid mechanics, 1987. 176: p. 379-402.

103. Champoux, Y. and J.F. Allard, Dynamic tortuosity and bulk modulus in air-saturated porous media. Journal of applied physics, 1991. 70(4): p. 1975-1979.

104. Motsinger, R. and R. Kraft, Design and performance of duct acoustic treatment. 1991.

105. Lee, T., T. Nomura, and H. Iizuka, Damped resonance for broadband acoustic absorption in one-port and two-port systems. Sci Rep, 2019. 9(1): p. 13077.

106. Merkel, A., et al., Control of acoustic absorption in one-dimensional scattering by resonant scatterers. Applied Physics Letters, 2015. 107(24).

107. Poirier, B., C. Maury, and J.-M. Ville, The use of Herschel-Quincke tubes to improve the efficiency of lined ducts. Applied acoustics, 2011. 72(2-3): p. 78-88.

108. Poirier, B., et al., Bicylindrical model of Herschel-Quincke tube-duct system: Theory and comparison with experiment and finite element method. The Journal of the Acoustical Society of America, 2009. 126(3): p. $1151-1162$. 
109. Burdisso, R. and J. Smith. Control of inlet noise from turbofan engines using Herschel-Quincke waveguides. in 6th Aeroacoustics Conference and Exhibit. 2000.

110. Maa, D.-Y., Potential of microperforated panel absorber. The Journal of the Acoustical Society of America, 1998. 104(5): p. 2861-2866.

111. Zhu, Y., et al., Ultrathin Acoustic Metasurface-Based Schroeder Diffuser. Physical Review X, 2017. 7(2).

112. Selamet, A. and I. Lee, Helmholtz resonator with extended neck. J. Acoust. Soc. Am., 2003. 113(4): p. 1975-1985.

113. Chen, S., et al., Engineering Coiling-Up Space Metasurfaces for Broadband Low-Frequency Acoustic Absorption. physica status solidi (RRL)-Rapid Research Letters, 2019. 13(12): p. 1900426.

114. Li, Y., S. Qi, and M.B. Assouar, Theory of metascreen-based acoustic passive phased array. New Journal of Physics, 2016. 18(4). 


\section{VITA}

Huy Nguyen was born in 1981 in Hanoi, Vietnam. He obtained his Engineer degree in Mechatronics and Master's degree in Engineering Mechanics, both from Hanoi University of Science and Technology, Vietnam. He started his Ph.D. program in the Department of Mechanical and Aerospace Engineering at the University of Missouri-Columbia in August 2014. 